\title{
EXISTENCE OF ORTHOGONAL GEODESIC CHORDS ON RIEMANNIAN MANIFOLDS WITH CONCAVE BOUNDARY AND HOMEOMORPHIC TO THE $N$-DIMENSIONAL DISK
}

\author{
ROBERTO GIAMBÒ, FABIO GIANNONI AND PAOLO PICCIONE
}

\begin{abstract}
In this paper we give a proof of the existence of an orthogonal geodesic chord on a Riemannian manifold homeomorphic to a closed disk and with concave boundary. This kind of study is motivated by the link (proved in [6]) of the multiplicity problem with the famous Seifert conjecture (formulated in 1948 (ref. [17) about multiple brake orbits for a class of Hamiltonian systems at a fixed energy level.
\end{abstract}

\section{CONTENTS}

1. Introduction................................................................................. 1

1.1. Brake orbits for a Hamiltonian system and statement of the Seifert conjecture...................................................... 2

1.2. Geodesics in Riemannian Manifolds with Boundary................. 3

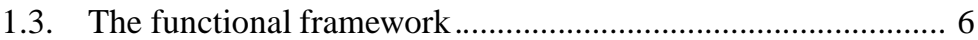

2. Path space and maximal intervals ................................................... 7

3. Geometrically critical values and variationally critical portions ....... 9

4. Classification of variationally critical portions .................................... 11

5. Some results about critical portions with respect to $\mathcal{V}^{-}$................... 14

6. The Palais-Smale condition for $\mathcal{V}^{+}$-variationally critical portion.... 17

7. The Palais-Smale for $\mathcal{V}^{-}$-critical portions and the notion of topological non essential interval ............................................... 23

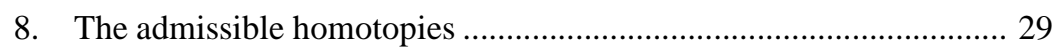

9. A deformation lemma with homotopies of type A ............................ 31

10. Deformation results for homotopies of type B .............................. 45

11. A deformation result for homotopies of type $\mathrm{C}$ and the First Defor-

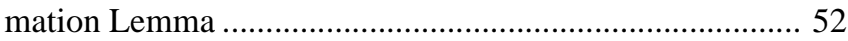

12. Proof of the main Theorem ............................................................... 55

Appendix A. Computation of the relative category ............................... 56

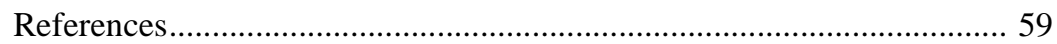

\section{INTRODUCTION}

We first recall the famous conjecture formulated by H. Seifert in 1948 (ref. [17]) concerning the number of brake orbits of a certain Hamiltonian system in $\mathbb{R}^{2 N}$.

Date: March 3rd, 2010 (revised version).

2000 Mathematics Subject Classification. 37C29, 37J45, 58E10. 
1.1. Brake orbits for a Hamiltonian system and statement of the Seifert conjecture. Denote by $(q, p)=\left(q_{1}, \ldots, q_{N}, p_{1}, \ldots, p_{N}\right)$ the canonical coordinates in $\mathbb{R}^{2 N}$, and consider a $C^{2}$ Hamiltonian function $H: \mathbb{R}^{2 N} \rightarrow \mathbb{R}$ of the form

$$
H(q, p)=\frac{1}{2} \sum_{i, j=1}^{N} a^{i j}(q) p_{i} p_{j}+V(q),
$$

where $q \mapsto\left(a^{i j}(q)\right)_{i, j}$ is a map of class $C^{2}$ taking value in the space of (symmetric) positive definite $N \times N$ matrices and $V: \mathbb{R}^{N} \rightarrow \mathbb{R}$ is the potential energy. The corresponding Hamiltonian system is:

$$
\left\{\begin{array}{l}
\dot{p}=-\frac{\partial H}{\partial q} \\
\dot{q}=\frac{\partial H}{\partial p}
\end{array}\right.
$$

where the dot denotes differentiation with respect to time. Since the system (1.2) is time independent, the total energy $E$ is constant along each solution, and there exists a large amount of literature concerning the study of periodic solutions of autonomous Hamiltonian systems with prescribed energy (see e.g. [14, 15] and references therein).

For all $q \in \mathbb{R}^{N}$, denote by $\mathcal{L}(q): \mathbb{R}^{N} \rightarrow \mathbb{R}^{N}$ the linear isomorphism whose matrix with respect to the canonical basis is $\left(a_{i j}(q)\right)$, the inverse of $\left(a^{i j}(q)\right)$. It is easily seen that, if $(q, p)$ is a $C^{1}$ solution of (1.2), then $q$ is actually a map of class $C^{2}$ and

$$
p=\mathcal{L}(q) \dot{q} .
$$

A special class of periodic solutions of (1.2) consists of the so called brake orbits. A brake orbit for the system (1.2) is a nonconstant solution $(q, p): \mathbb{R} \rightarrow \mathbb{R}^{2 N}$ such that $p(0)=p(T)=0$ for some $T>0$; since $H$ is even in the momenta $p$, then a brake orbit is $2 T$-periodic. Moreover, if $E$ is the energy of a brake orbit $(q, p)$, then $V(q(0))=V(q(T))=E$.

Let $E>\inf V$ be fixed; we will make the following assumptions:

$$
E \text { is a regular value of } V \text {, namely } \mathrm{d} V(q) \neq 0 \text { for all } q \in V^{-1}(E) \text {, }
$$

$$
\text { the closed sublevel } \left.\left.V^{-1}(]-\infty, E\right]\right) \text { is homeomorphic to the } N \text {-disk } \mathbb{D}^{N} \text {, }
$$

(here $\mathrm{d} V(q)$ denotes the differential of $V$ ). We refer the reader e.g. to [2, 10, 14, 19, 20] for multiplicity results of brake orbits.

In [2] and [10], under assumptions (1.4) and (1.5), it is proved the existence of at least $N$ brake orbits, but using a very strong (ad hoc) assumption on the energy integral, to ensure that different minimax critical levels correspond to geometrically distinct brake orbits. (By geometrically distinct curves we mean curves whose images are distinct sets).

Papers [14, 19, 20] deal with more general Hamiltonian systems not necessarily of the form (1.1). In [19] it is proved that if $H$ is even in the variable $p$, and $H^{-1}(1)$ bounds a starshaped region and satisfies a suitable geometric condition (that allows once again to obtain geometrically distinct brake orbits), then there are at least $N$ brake orbits on $H^{-1}(1)$. In [20] the author prove that for any $h$ sufficiently close to $H\left(z_{0}\right)$ with $z_{0}$ nondegenerate local minimum for $H$, there are $N$ geometrically distinct brake orbits in $H^{-1}(h)$. This result generalize (in the case of a minimum point) a classical theorem of Lyapunov for nondegenerate critical points having the eigenvalues of the Hessian matrix with non integer ratio.

In [14] if $H$ is convex and even in both variables $p$ and $q$ the authors prove the existence of at least 2 geometrically distinct brake orbits on $H^{-1}(1)$, provided that it is regular and compact. 
In the paper [17], under assumptions (1.4) and (1.5), Seifert proved the existence of a brake orbit, and he also conjectured the existence of at least $N$ geometrically distinct brake orbits. Up to the present day, the conjecture has been neither proved nor disproved.

It is well known that the lower estimate for the number of brake orbits given in the Seifert conjecture cannot be improved in any dimension $N$. Indeed, consider the Hamiltonian:

$$
H(q, p)=\frac{1}{2}|p|^{2}+\sum_{i=1}^{N} \lambda_{i}^{2} q_{i}^{2}, \quad(q, p) \in \mathbb{R}^{2 N},
$$

where $\lambda_{i} \neq 0$ for all $i$. If $E>0$ and the squared ratios $\left(\lambda_{i} / \lambda_{j}\right)^{2}$ are irrational for all $i \neq j$, then the only periodic solutions of (1.2) with energy $E$ are the $N$ brake orbits moving along the axes of the ellipsoid with equation

$$
\sum_{i=1}^{N} \lambda_{i}^{2} q_{i}^{2}=E
$$

In [6] it has been pointed out that the problem of finding brake orbits is equivalent to find orthogonal geodesic chords on manifold with concave boundary.

In this paper we use a suitable functional approach to prove the existence of one orthogonal geodesic chords for a manifold with concave boundary homeomorphic to the $N$-dimensional disk. This is the first step in view of obtaining multiplicity results for orthogonal geodesic chords in this kind of situation, and a new way to study Seifert conjecture.

Let us now recall a few basic facts and notations from [6].

1.2. Geodesics in Riemannian Manifolds with Boundary. Let $(M, g)$ be a smooth Riemannian manifold with $\operatorname{dim}(M)=N \geq 2$. The symbol $\nabla$ will denote the covariant derivative of the Levi-Civita connection of $g$, as well as the gradient differential operator for smooth maps on $M$. The Hessian $\mathrm{H}^{f}(q)$ of a smooth map $f: M \rightarrow \mathbb{R}$ at a point $q \in M$ is the symmetric bilinear form $\mathrm{H}^{f}(q)(v, w)=g\left(\left(\nabla_{v} \nabla f\right)(q), w\right)$ for all $v, w \in T_{q} M$; equivalently, $\mathrm{H}^{f}(q)(v, v)=\left.\frac{\mathrm{d}^{2}}{\mathrm{~d} s^{2}}\right|_{s=0} f(\gamma(s))$, where $\left.\gamma:\right]-\varepsilon, \varepsilon[\rightarrow M$ is the unique (affinely parameterized) geodesic in $M$ with $\gamma(0)=q$ and $\dot{\gamma}(0)=v$. We will denote by $\frac{\mathrm{D}}{\mathrm{d} t}$ the covariant derivative along a curve, in such a way that $\frac{\mathrm{D}}{\mathrm{d} t} \dot{x}=0$ is the equation of the geodesics. A basic reference on the background material for Riemannian geometry is [4].

Let $\Omega \subset M$ be an open subset; $\bar{\Omega}=\Omega \bigcup \partial \Omega$ will denote its closure. There are several notion of convexity and concavity in Riemannian geometry, extending the usual ones for subsets of the Euclidean space $\mathbb{R}^{N}$. In this paper we will use a somewhat strong concavity assumption for $\bar{\Omega}$, that we will call "strong concavity" below, and which is stable by $C^{2}$ small perturbations of the boundary. Let us first recall the following definition (cf also [1], 8]):

Definition 1.1. $\bar{\Omega}$ is said to be convex if every geodesic $\gamma:[a, b] \rightarrow \bar{\Omega}$ whose endpoints $\gamma(a)$ and $\gamma(b)$ are in $\Omega$ has image entirely contained in $\Omega$. Likewise, $\bar{\Omega}$ is said to be concave if its complement $M \backslash \bar{\Omega}$ is convex.

If $\partial \Omega$ is a smooth embedded submanifold of $M$, let $\mathbb{I}_{\mathfrak{n}}(x): T_{x}(\partial \Omega) \times T_{x}(\partial \Omega) \rightarrow \mathbb{R}$ denote the second fundamental form of $\partial \Omega$ in the normal direction $\mathfrak{n} \in T_{x}(\partial \Omega)^{\perp}$. Recall that $\mathbb{I}_{\mathfrak{n}}(x)$ is a symmetric bilinear form on $T_{x}(\partial \Omega)$ defined by:

$$
\mathbb{I}_{\mathfrak{n}}(x)(v, w)=g\left(\nabla_{v} W, \mathfrak{n}\right), \quad v, w \in T_{x}(\partial \Omega),
$$

where $W$ is any local extension of $w$ to a smooth vector field along $\partial \Omega$. 
Remark 1.2. Assume that it is given a smooth function $\phi: M \rightarrow \mathbb{R}$ with the property that $\Omega=\phi^{-1}(]-\infty, 0[)$ and $\partial \Omega=\phi^{-1}(0)$, with $\nabla \phi \neq 0$ on $\partial \Omega$. The following equality between the Hessian $\mathrm{H}^{\phi}$ and the second fundamental form 2 of $\partial \Omega$ holds:

$$
\mathrm{H}^{\phi}(x)(v, v)=-\mathbb{I}_{\nabla \phi(x)}(x)(v, v), \quad x \in \partial \Omega, v \in T_{x}(\partial \Omega) ;
$$

Namely, if $x \in \partial \Omega, v \in T_{x}(\partial \Omega)$ and $V$ is a local extension around $x$ of $v$ to a vector field which is tangent to $\partial \Omega$, then $v(g(\nabla \phi, V))=0$ on $\partial \Omega$, and thus:

$$
\mathrm{H}^{\phi}(x)(v, v)=v(g(\nabla \phi, V))-g\left(\nabla \phi, \nabla_{v} V\right)=-\mathbb{I}_{\nabla \phi(x)}(x)(v, v) .
$$

For convenience, we will fix throughout the paper a function $\phi$ as above. We observe that, although the second fundamental form is defined intrinsically, there is no a canonical choice for a function $\phi$ describing the boundary of $\Omega$ as above.

Definition 1.3. We will say that that $\bar{\Omega}$ is strongly concave if $\mathbb{I}_{\mathfrak{n}}(x)$ is negative definite for all $x \in \partial \Omega$ and all inward pointing normal direction $\mathfrak{n}$.

Note that if $\bar{\Omega}$ is strongly concave, then geodesics starting on $\partial \Omega$ tangentially to $\partial \Omega$ locally move inside $\Omega$.

Remark 1.4. Strong concavity is evidently a $C^{2}$-open condition. Then, if $\bar{\Omega}$ is compact, there exists $\delta_{0}>0$ such that $H^{\phi}(q)[v, v]<0$ for any $q$ such that $\phi(q) \in\left[-\delta_{0}, \delta_{0}\right]$ and $g(\nabla \phi(q), v)=0$. A simple contradiction argument based on Taylor expansion shows that under the above conditions there are not critical points of $\phi$ for all $q \in \phi^{-1}\left(\left[-\delta_{0}, \delta_{0}\right]\right)$. In particular the gradient flow of $\phi$ gives a strong deformation retract of $\phi^{-1}\left(\left[-\delta_{0}, \delta_{0}\right]\right)$ onto $\phi^{-1}(0)=\partial \Omega$.

Remark 1.5. The strong concavity condition gives us the following property, that will be systematically used throughout the paper. Let $\delta_{0}$ be as in Remark 1.4; then:

for any non constant geodesic $\gamma:[a, b] \rightarrow \bar{\Omega}$ with $\phi(\gamma(a)=\phi(\gamma(b))=0$ and $\phi(\gamma(s))<0 \forall s \in] a, b[$, there exists $\bar{s} \in] a, b\left[\right.$ such that $\phi(\gamma(\bar{s}))<-\delta_{0}$.

Such property is proved easily by a contradiction argument obtained by looking at the minimum point of the map $s \mapsto \phi(\gamma(s))$.

The main objects of our study are geodesics in $M$ having image in $\bar{\Omega}$ and with endpoints orthogonal to $\partial \Omega$, that will be called orthogonal geodesic chords:

Definition 1.6. A geodesic $\gamma:[a, b] \rightarrow M$ is called a geodesic chord in $\bar{\Omega}$ if $\gamma(] a, b[) \subset \Omega$ and $\gamma(a), \gamma(b) \in \partial \Omega$; by a weak geodesic chord we will mean a geodesic $\gamma:[a, b] \rightarrow$ $M$ with image in $\bar{\Omega}$, endpoints $\gamma(a), \gamma(b) \in \partial \Omega$ and such that $\gamma\left(s_{0}\right) \in \partial \Omega$ for some $\left.s_{0} \in\right] a, b\left[\right.$. A (weak) geodesic chord is called orthogonal if $\dot{\gamma}\left(a^{+}\right) \in\left(T_{\gamma(a)} \partial \Omega\right)^{\perp}$ and $\dot{\gamma}\left(b^{-}\right) \in\left(T_{\gamma(b)} \partial \Omega\right)^{\perp}$, where $\dot{\gamma}\left(\cdot{ }^{ \pm}\right)$denote the lateral derivatives (see Figure 1 ).

For shortness, we will write OGC for "orthogonal geodesic chord" and WOGC for "weak orthogonal geodesic chord". Although the general class of weak orthogonal geodesic chords are perfectly acceptable solutions of our initial geometrical problem, our suggested construction of a variational setup works well only in a situation where one can exclude $a$ priori the existence in $\bar{\Omega}$ of orthogonal geodesic chords $\gamma:[a, b] \rightarrow \bar{\Omega}$ for which there exists $\left.s_{0} \in\right] a, b\left[\right.$ such that $\gamma\left(s_{0}\right) \in \partial \Omega$.

One does not lose generality in assuming that there are no such WOGC's in $\bar{\Omega}$ by recalling the following result from [6]:

\footnotetext{
${ }^{1}$ For example one can choose $\phi$ such that $|\phi(q)|=\operatorname{dist}(q, \partial \Omega)$ for all $q$ in a (closed) neighborhood of $\partial \Omega$, where dist denotes the distance function on $M$ induced by $g$.

${ }^{2}$ Observe that, with our definition of $\phi, \nabla \phi$ is a normal vector to $\partial \Omega$ pointing outwards from $\Omega$.
} 


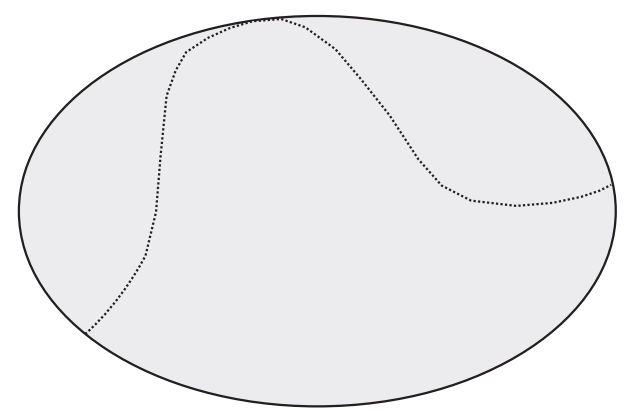

FIGURE 1. A weak orthogonal geodesic chord (WOGC) $\gamma$ in $\bar{\Omega}$.

Proposition 1.7. Let $\Omega \subset M$ be an open set whose boundary $\partial \Omega$ is smooth and compact and with $\bar{\Omega}$ strongly concave. Assume that there are only a finite number of orthogonal geodesic chords in $\bar{\Omega}$. Then, there exists an open subset $\Omega^{\prime} \subset \Omega$ with the following properties:

(1) $\overline{\Omega^{\prime}}$ is diffeomorphic to $\bar{\Omega}$ and it has smooth boundary;

(2) $\overline{\Omega^{\prime}}$ is strongly concave;

(3) the number of OGC's in $\overline{\Omega^{\prime}}$ is less than or equal to the number of OGC's in $\bar{\Omega}$;

(4) there are not WOGC's in $\overline{\Omega^{\prime}}$.

Proof. See [6, Proposition 2.6]

In the central result of this paper we shall give the proof of the existence of at least an OGC. Note that, whenever $\partial \Omega$ is not connected the existence problem has a very simple solution without assumptions on the geometry of $\bar{\Omega}$. Indeed it suffices to minimize the energy functional on the curves joining two different components of $\partial \Omega$. Whenever $\partial \Omega$ is connected the problem in general has no solution. A simple and famous counterexample can be found in [及].

The situation is different if we assume $\bar{\Omega}$ to be convex or concave. In the convex case we wish first to recall the result proved by Bos in [ [ $]$ : if $\partial \Omega$ is smooth, $\bar{\Omega}$ convex and homeomorphic to the $N$-dimensional disk, then there are at least $N$ geometrically distinct OGC's for $\bar{\Omega}$. Such a result is a generalization of the classical one of Ljusternik and Schnirelman (see [16]), where they treated convex subsets of $\mathbb{R}^{N}$ endowed with the Euclidean metric. Always in the convex case, in [⿰] it is studied the effect of the topology of $\bar{\Omega}$ on the number of OGC's.

In this paper we prove the existence result in the concave case:

Theorem. Let $\Omega$ be an open subset of $M$ with smooth boundary $\partial \Omega$, such that $\bar{\Omega}$ is strongly concave and homeomorphic to an $N$-dimensional disk. Suppose that there are not WOGC's. Then there is at least an orthogonal geodesic chord in $\bar{\Omega}$.

Existence and multiplicity of OGC's in the case of compact manifolds having convex boundary is typically proven by applying a curve-shortening argument. From an abstract viewpoint, the curve-shortening process can be seen as the construction of a flow in the space of paths, along whose trajectories the length or energy functional is decreasing.

In this paper we will follow the same procedure, with the difference that both the space of paths and the shortening flow have to be defined appropriately.

Shortening a curve having image in a closed convex subset $\bar{\Omega}$ of a Riemannian manifold produces another curve in $\bar{\Omega}$; in this sense, we think of the shortening flow as being "inward pushing" in the convex case. As opposite to the convex case, the shortening flow in the concave case will be "outwards pushing", and this fact requires that one should consider only those portions of a curve that remain inside $\bar{\Omega}$ when it is stretched outwards. 
Unfortunately this kind of approach produces a lot of technical difficulties. For this reason we shall try to describe the main ideas of the paper before to face the main difficulties.

Obviously the concavity condition plays a central role in the variational setup of our construction. "Variational criticality" relatively to the energy functional will be defined in terms of "outwards pushing" infinitesimal deformations of the path space (see Definition 3.3. The class of variationally critical portions contains properly the set of portions of paths consisting of OGC's. In order to construct the shortening flow, an accurate analysis of all possible variationally critical portions is required (Section 4 ), and the concavity condition will guarantee that such paths are well behaved (see Lemma 4.1, Proposition 4.2 and Proposition 4.3). Thanks their good behavior, it will be possible to move far away from critical portions which are not OGC's (choosing a suitable class of admissible homotopies).

1.3. The functional framework. Throughout the paper, $(M, g)$ will denote a Riemannian manifold of class $C^{2}$; all our constructions will be made in suitable (relatively) compact subsets of $M$, and for this reason it will not be restrictive to assume, as we will, that $(M, g)$ is complete. Furthermore, we will work mainly in open subsets $\Omega$ of $M$ whose closure is homeomorphic to an $N$-dimensional disk, and in order to simplify the exposition we will assume that, indeed, $\bar{\Omega}$ is embedded topologically in $\mathbb{R}^{N}$, which will allow to use an auxiliary linear structure on a neighborhood of $\bar{\Omega}$. We will also assume that $\bar{\Omega}$ is strongly concave in $M$.

Recall that we have fixed a smooth function $\phi: M \rightarrow \mathbb{R}$ such that $\Omega=\phi^{-1}(]-\infty, 0[)$, $\nabla \phi \neq 0$ on $\partial \Omega$. The strong concavity of $\bar{\Omega}$ means that the Hessian $\mathrm{H}^{\phi}$ is negative definite on $T(\partial \Omega)$. As observed in Remark 1.4 there exists $\delta_{0}>0$ such that $\nabla \phi \neq 0$ and $H^{\phi}$ is negative definite in $\phi^{-1}\left(\left[-\delta_{0}, \delta_{0}\right]\right)$.

The symbol $H^{1}\left([a, b], \mathbb{R}^{N}\right)$ will denote the Sobolev space of all absolutely continuous curves $x:[a, b] \rightarrow \mathbb{R}^{N}$ whose weak derivative is square integrable. By $H_{0}^{1}\left([a, b], \mathbb{R}^{N}\right)$ we will denote the subspace of $H^{1}\left([a, b], \mathbb{R}^{N}\right)$ consisting of curves $x$ such that $x(a)=$ $x(b)=0$. The Hilbert space norm of $H^{1}\left([a, b], \mathbb{R}^{N}\right)$ will be denoted by $\|\cdot\|_{a, b}$ :

$$
\|x\|_{a, b}=\frac{1}{\sqrt{2}}\left(\max \left(\|x(a)\|_{E}^{2},\|x(b)\|_{E}^{2}\right)+\int_{a}^{b}\|\dot{x}\|_{E}^{2}\right)^{1 / 2}
$$

where $\|\cdot\|_{E}$ denotes the Euclidean norm. Note that a simple computation shows that the above norm is equivalent to the usual one in $H^{1}\left([a, b], \mathbb{R}^{N}\right)$. Note also that, setting

$$
\|x\|_{L^{\infty}\left([a, b], \mathbb{R}^{N}\right)}=\sup _{s \in[a, b]}\|x(s)\|_{E},
$$

the following inequality holds:

$$
\|x\|_{L^{\infty}\left([a, b], \mathbb{R}^{N}\right)} \leq\|x\|_{a, b}
$$

We shall use also the space $H^{2, \infty}$ which consists of curves having second derivative in $L^{\infty}$.

Remark 1.8. In the development of our results, we will have to deal with curves $x$ with variable domains $[a, b] \subset[0,1]$. In this situation, by $H^{1}$-convergence (resp., weak $H^{1}$ convergence, uniform convergence) of a sequence $x_{n}:\left[a_{n}, b_{n}\right] \rightarrow M$ to a curve $x$ : $[a, b] \rightarrow M$ we will mean that $a_{n}$ tends to $a, b_{n}$ tends to $b$ and $\widehat{x}_{n}:[a, b] \rightarrow M$ is $H^{1}$ convergent (resp., weakly $H^{1}$-convergent, uniformly convergent) to $x$ on $[a, b]$ as $n \rightarrow \infty$, where $\widehat{x}_{n}$ is the unique affine reparameterization of $x$ on the interval $[a, b]$. 


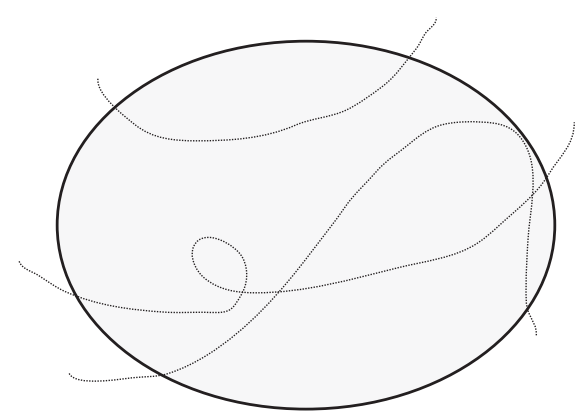

FIGURE 2. Curves representing typical elements of the path space $\mathfrak{M}_{0}$.

In the rest of the paper it will be useful to consider the flows $\eta^{+}(\tau, x)$ and $\eta^{-}(\tau, x)$ on the Riemannian manifold $M$ defined by

$$
\left\{\begin{array}{l}
\frac{\mathrm{d}}{\mathrm{d} \tau} \eta^{+}(\tau)=\frac{\nabla \phi\left(\eta^{+}\right)}{\left\|\nabla \phi\left(\eta^{+}\right)\right\|^{2}} \\
\eta^{+}(0)=x \in\left\{y \in M:-\delta_{0} \leq \phi(y) \leq \delta_{0}\right\},
\end{array}\right.
$$

and

$$
\left\{\begin{array}{l}
\frac{\mathrm{d}}{\mathrm{d} \tau} \eta^{-}(\tau)=\frac{-\nabla \phi\left(\eta^{-}\right)}{\left\|\nabla \phi\left(\eta^{-}\right)\right\|^{2}} \\
\eta^{-}(0)=x \in\left\{y \in M:-\delta_{0} \leq \phi(y) \leq \delta_{0}\right\} .
\end{array}\right.
$$

Note that $\eta^{+}(\tau, x)$ and $\eta^{-}(\tau, x)$ are certainly well defined on $\phi^{-1}\left(\left[-\delta_{0}, \delta_{0}\right]\right)$, where $\nabla \phi \neq 0$.

We conclude this section with the introduction of the following constant:

$$
K_{0}=\max _{\left.x \in \phi^{-1}(]-\infty, \delta_{0}\right]}\|\nabla \phi(x)\|,
$$

where $\|\cdot\|$ is the norm induced by $g$, and $\delta_{0}$ is as in Remark 1.4 .

\section{PATH SPACE AND MAXIMAL INTERVALS}

In this section we shall describe the space of curves $\mathfrak{M}$ which will be the environment of our minimax approach and the set $\mathfrak{C} \subset \mathfrak{M}$ homeomorphic to $\mathbb{S}^{N-1} \times \mathbb{S}^{N-1}$, carrying on all the topological information of $\mathfrak{M}$.

For $\mathcal{C} \subset \mathbb{R}^{N}$ and $a<b$, define:

$$
H^{1}([a, b], \mathcal{C})=\left\{x \in H^{1}\left([a, b], \mathbb{R}^{N}\right): x(s) \in \mathcal{C} \text { for all } s \in[a, b]\right\} .
$$

Let $\delta_{0}>0$ be as in Remark 1.4. Consider first the following set of paths

$$
\mathfrak{M}_{0}=\left\{x \in H^{1}\left([0,1], \phi^{-1}(]-\infty, \delta_{0}[)\right): \phi(x(0)) \geq 0, \phi(x(1)) \geq 0\right\},
$$

see Figure 2. This is a subset of the Hilbert space $H^{1}\left([0,1], \mathbb{R}^{N}\right)$, and it will be topologized with the induced metric.

The following result will be used systematically throughout the paper:

Lemma 2.1. If $x \in \mathfrak{M}_{0}$ and $[a, b] \subset[0,1]$ is such that $x(a) \in \partial \Omega$ and there exists $\bar{s} \in[a, b]$ such that $\phi(x(\bar{s})) \leq-\delta<0$, then:

$$
b-a \geq \frac{\delta^{2}}{K_{0}^{2}}\left(\int_{a}^{b} g(\dot{x}, \dot{x}) \mathrm{d} s\right)^{-1},
$$


where $K_{0}$ is defined in (1.12).

Proof. Since $\phi(x(a))=0$, if $\bar{s}$ is such that $\phi(x(\bar{s}))=-\delta<0$, it is:

$$
\begin{gathered}
\delta \leq|\phi(x(\bar{s}))-\phi(x(a))| \leq \int_{a}^{\bar{s}}|g(\nabla \phi(x(\sigma)), \dot{x}(\sigma))| \mathrm{d} \sigma \leq \int_{a}^{b}|g(\nabla \phi(x(\sigma)), \dot{x}(\sigma))| \mathrm{d} \sigma \\
\leq K_{0} \int_{a}^{b} g(\dot{x}, \dot{x})^{\frac{1}{2}} \mathrm{~d} \sigma \leq K_{0} \sqrt{b-a}\left(\int_{a}^{b} g(\dot{x}, \dot{x}) \mathrm{d} \sigma\right)^{\frac{1}{2}},
\end{gathered}
$$

from which (2.3) is easily deduced.

For all $x \in \mathfrak{M}_{0}$, let $\mathcal{I}_{x}^{0}, \mathcal{I}_{x}$ denote the following collections of closed subintervals of $[0,1]$ :

$$
\begin{gathered}
\mathcal{I}_{x}^{0}=\{[a, b] \subset[0,1]: x([a, b]) \subset \bar{\Omega}, x(a), x(b) \in \partial \Omega\}, \\
\mathcal{I}_{x}=\left\{[a, b] \in \mathcal{I}_{x}^{0} \text { and }[a, b] \text { is maximal with respect to this property }\right\} .
\end{gathered}
$$

Although we will only prove an existence result, in this paper we will employ an equivariant approach that aims also at multiplicity results, to be developed in the future.

To this aim we shall consider the map $\mathcal{R}: \mathfrak{M}_{0} \rightarrow \mathfrak{M}_{0}$ :

$$
\mathcal{R} x(t)=x(1-t) .
$$

We say that $\mathfrak{N} \subset \mathfrak{M}_{0}$ is $\mathcal{R}$-invariant if $\mathcal{R}(\mathfrak{N})=\mathfrak{N}$; note that $\mathfrak{M}_{0}$ is $\mathcal{R}$-invariant. If $\mathfrak{N}$ is $\mathcal{R}$-invariant, a homotopy $h:[0,1] \times \mathfrak{N} \rightarrow \mathfrak{M}_{0}$ is called $\mathcal{R}$-equivariant if

$$
h(\tau, \mathcal{R} x)=\mathcal{R} h(\tau, x), \quad \forall x \in \mathfrak{N}, \forall \tau \in[0,1] .
$$

The following Lemma allows to describe an $\mathcal{R}$-invariant subset $\mathfrak{C}$ of $\mathfrak{M}_{0}$ which carries on all the topological properties of $\mathfrak{M}_{0}$.

Lemma 2.2. There exists there a continuous map $G: \partial \Omega \times \partial \Omega \rightarrow H^{1}([0,1], \bar{\Omega})$ such that

(1) $G(A, B)(0)=A, G(A, B)(1)=B$;

(2) $A \neq B \Rightarrow G(A, B)(s) \in \Omega \forall s \in] 0,1[$;

(3) $G(A, A)(s)=A \forall s \in[0,1]$;

(4) $\mathcal{R} G(A, B)=G(B, A)$, namely $G(A, B)(1-s)=G(B, A)(s)$ for all $s$, and for all $A, B$.

Proof. If $\bar{\Omega}$ is diffeomorphic to the $N$-dimensional disk $\mathbb{D}^{N}=\left\{q \in \mathbb{R}^{N}:\|q\|_{E} \leq 1\right\}$ and $\psi: \bar{\Omega} \rightarrow \mathbb{D}^{N}$ is a diffeomorphism, we set

$$
G(A, B)(s)=\psi^{-1}((1-s) \psi(A)+s \psi(B)), A, B \in \bar{\Omega} .
$$

In general, if $\bar{\Omega}$ is only homeomorphic to the disk $\mathbb{D}^{N}$, then the above definition produces curves that in principle are only continuous. In order to produce curves with an $H^{1}$-regularity, we use a broken geodesic approximation argument. More precisely, choose a homeomorphism $\psi: \bar{\Omega} \rightarrow \mathbb{D}^{N}$ and consider for any $A, B \in \bar{\Omega}$ the curve

$$
c_{A, B}(s)=\psi^{-1}((1-s) \psi(A)+s \psi(B))
$$

and the set

$$
\mathfrak{C}^{\prime}=\left\{c_{A, B}: A, B \in \bar{\Omega}\right\} .
$$

Denote by $\varrho(\bar{\Omega}, g)$ the infimum of the injectivity radii of all points of $\bar{\Omega}$ relatively to the metric $g$ (cf. [丹]). By the compactness of $\mathfrak{C}^{\prime}$, there exists $N_{0} \in \mathbb{N}$ with the property that $\operatorname{dist}\left(c_{A, B}(a), c_{A, B}(b)\right) \leq \varrho(\bar{\Omega}, g)$ whenever $|a-b| \leq \frac{1}{N_{0}}$ for all $c_{A, B} \in \mathfrak{C}^{\prime}$. Finally, for all $c_{A, B} \in \mathfrak{C}^{\prime}$, denote by $\gamma_{A, B}$ the broken geodesic obtained as concatenation of the curves $\gamma_{k}:\left[\frac{k-1}{N_{0}}, \frac{k}{N_{0}}\right] \rightarrow M$ given by the unique minimal geodesic in $(M, g)$ from $c_{A, B}\left(\frac{k-1}{N_{0}}\right)$ to $c_{A, B}\left(\frac{k}{N_{0}}\right), k=1, \ldots, N_{0}$. 
Note that, by compactness, $N_{0}$ can be chosen large enough to have

$$
\phi\left(\gamma_{A, B}(s)\right)<\delta_{0} \text { for any } s \in[0,1] \text {, for any } A, B \in \bar{\Omega} .
$$

Since the minimal geodesic in any convex normal neighborhood depend continuously (with respect to the $C^{2}$-norm) on its endpoints, $\gamma_{A, B}$ depends continuously by $(A, B)$ in the $H^{1}$-norm. Moreover $\gamma_{A, B}$ satisfies (1), (3) and (4) of the statement.

Now, using the flow $\eta^{-}$defined by (1.11), we can set

$$
\hat{\gamma}(A, B)(s)=\eta^{-}\left(\max \left(0, \phi\left(\gamma_{A, B}(s)\right), \gamma_{A, B}(s)\right),\right.
$$

so that

$$
\hat{\gamma}(A, B)(s) \in \bar{\Omega} \text { for any } s \in[0,1] \text {, for any } A, B \in \bar{\Omega} \text {. }
$$

Since $\gamma(A, B)(0)=A$ and $\gamma(A, B)(1)=B$, it is $\phi(\gamma(A, B)(0))=\phi(\gamma(A, B)(1))=0$ and therefore also $\hat{\gamma}_{A, B}$ satisfies (1), (3) and (4) of the statement. Finally,

$$
G(A, B)(s)=\eta^{-}\left(s(1-s) \max \left(0, \frac{\delta_{0}}{2}+\phi\left(\hat{\gamma}_{A, B}(s)\right), \hat{\gamma}_{A, B}(s)\right),\right.
$$

is the desired map.

We set

$$
\begin{aligned}
& \mathfrak{C}=\{G(A, B): A, B \in \partial \Omega\}, \\
& \mathfrak{C}_{0}=\{G(A, A): A \in \partial \Omega\} .
\end{aligned}
$$

Remark 2.3. Note that $\mathfrak{C}$ is homeomorphic to $\mathbb{S}^{N-1} \times \mathbb{S}^{N-1}$ by a homeomorphism mapping $\mathfrak{C}_{0}$ onto $\left\{(A, A): A \in \mathbb{S}^{N-1}\right\}$, and that $\mathcal{R}$ induces (by such homeomorphism) an action $\mathcal{S}$ on $\mathbb{S}^{N-1} \times \mathbb{S}^{N-1}$ given by

$$
\mathcal{S}(A, B)=(B, A)
$$

Define now the following constant:

$$
M_{0}=\sup _{x \in \mathfrak{C}} \int_{0}^{1} g(\dot{x}, \dot{x}) \mathrm{d} t .
$$

Since $\mathfrak{C}$ is compact and the integral in $(2.8)$ is continuous in the $H^{1}$-topology, then $M_{0}<$ $+\infty$. Finally we define the following subset of $\mathfrak{M}_{0}$ :

$$
\mathfrak{M}=\left\{x \in \mathfrak{M}_{0}: \frac{1}{2} \int_{a}^{b} g(\dot{x}, \dot{x}) \mathrm{d} t<M_{0} \quad \forall[a, b] \in \mathcal{I}_{x}\right\} .
$$

We shall work in $\mathfrak{M}$ constructing flows in $H^{1}\left([0,1], \mathbb{R}^{N}\right)$ for which $\mathfrak{M}$ is invariant. We shall often use the notation

$$
f_{a, b}(x)=\frac{1}{2} \int_{a}^{b} g(\dot{x}, \dot{x}) \mathrm{d} t .
$$

\section{GeOMETRICALLY CRITICAL VALUES AND VARIATIONALLY CRITICAL PORTIONS}

In this section we will introduce two different notions of criticality for curves in $\mathfrak{M}$.

Definition 3.1. A number $c \in] 0, M_{0}$ [ will be called a geometrically critical value if there exists an OGC $\gamma$ parameterized in $[0,1]$ such that $\frac{1}{2} \int_{0}^{1} g(\dot{\gamma}, \dot{\gamma}) \mathrm{d} t=c$. A number which is not geometrically critical will be called geometrically regular value.

It is important to observe that, in view to obtain multiplicity results, distinct geometrically critical values yield geometrically distinct orthogonal geodesic chords:

Proposition 3.2. Let $c_{1} \neq c_{2}, c_{1}, c_{2}>0$ be distinct geometrically critical values with corresponding OGC $x_{1}, x_{2}$. Then $x_{1}([0,1]) \neq x_{2}([0,1])$. 
Proof. The OGC's $x_{1}$ and $x_{2}$ are parameterized in the interval $[0,1]$. Assume by contradiction, $x_{1}([0,1])=x_{2}([0,1])$. Since

$$
x_{i}(] 0,1[) \subset \Omega \text { for any } i=1,2,
$$

we have

$$
\left\{x_{1}(0), x_{1}(1)\right\}=\left\{x_{2}(0), x_{2}(1)\right\} .
$$

Up to reversing the orientation of $x_{2}$, we can assume $x_{1}(0)=x_{2}(0)$. Since $x_{1}$ and $x_{2}$ are OGC's, $\dot{x}_{1}(0)$ and $\dot{x}_{2}(0)$ are parallel, but the condition $c_{1} \neq c_{2}$ says that $\dot{x}_{1}(0) \neq \dot{x}_{2}(0)$. Then there exists $\lambda>0, \lambda \neq 1$ such that $\dot{x}_{2}(0)=\lambda \dot{x}_{1}(0)$ and therefore, by the uniqueness of the Cauchy problem for geodesics we have $x_{2}(s)=x_{1}(\lambda s)$. Up to exchanging $x_{1}$ with $x_{2}$, we can assume $\lambda>1$. Since $x_{2}\left(\frac{1}{\lambda}\right)=x_{1}(1) \in \partial \Omega$, the transversality of $\dot{x}_{2}(0)$ to $\partial \Omega$ implies the existence of $\left.\bar{s} \in] \frac{1}{\lambda}, 1\right]$ such that $x_{2}(\bar{s}) \notin \bar{\Omega}$, getting a contradiction.

A notion of criticality will now be given in terms of variational vector fields. For $x \in \mathfrak{M}$, let $\mathcal{V}^{+}(x)$ denote the following closed convex cone of $T_{x} H^{1}\left([0,1], \mathbb{R}^{N}\right)$ :

$$
\mathcal{V}^{+}(x)=\left\{V \in T_{x} H^{1}\left([0,1], \mathbb{R}^{N}\right): g(V(s), \nabla \phi(x(s))) \geq 0 \text { for } x(s) \in \partial \Omega\right\}
$$

vector fields in $\mathcal{V}^{+}(x)$ are interpreted as infinitesimal variations of $x$ by curves stretching "outwards" from the set $\bar{\Omega}$.

Definition 3.3. Let $x \in \mathfrak{M}$ and $[a, b] \subset[0,1]$; we say that $x_{\mid[a, b]}$ is a $\mathcal{V}^{+}$-variationally critical portion of $x$ if $x_{[[a, b]}$ is not constant and if

$$
\int_{a}^{b} g\left(\dot{x}, \frac{\mathrm{D}}{\mathrm{d} t} V\right) \mathrm{d} t \geq 0, \quad \forall V \in \mathcal{V}^{+}(x) .
$$

Similarly, for $x \in \mathfrak{M}$ we define the cone:

$$
\mathcal{V}^{-}(x)=\left\{V \in T_{x} H^{1}\left([0,1], \mathbb{R}^{N}\right): g(V(s), \nabla \phi(x(s))) \leq 0 \text { for } x(s) \in \partial \Omega\right\},
$$

and we give the following

Definition 3.4. Let $x \in \mathfrak{M}$ and $[a, b] \subset[0,1]$; we say that $x_{\mid[a, b]}$ is a $\mathcal{V}^{-}$-variationally critical portion of $x$ if $x_{[[a, b]}$ is not constant and if

$$
\int_{a}^{b} g\left(\dot{x}, \frac{\mathrm{D}}{\mathrm{d} t} V\right) \mathrm{d} t \geq 0, \quad \forall V \in \mathcal{V}^{-}(x) .
$$

The integral in (3.4) gives precisely the first variation of the geodesic action functional in $(M, g)$ along $x_{\mid[a, b]}$. Hence, variationally critical portions are interpreted as those curves $x_{\mid[a, b]}$ whose geodesic energy is not decreased after infinitesimal variations by curves stretching outwards from the set $\bar{\Omega}$. The motivation for using outwards pushing infinitesimal variations is due to the concavity of $\bar{\Omega}$. Indeed in the convex case it is customary to use a curve shortening method in $\bar{\Omega}$, that can be seen as the use of a flow constructed by infinitesimal variations of $x$ in $\mathcal{V}^{-}(x)$, keeping the endpoints of $x$ on $\partial \Omega$.

Flows obtained as integral flows of convex combinations of vector fields in $\mathcal{V}^{+}(x)$ are, in a certain sense, the protagonists of our variational approach. However we shall use also integral flows of convex combinations of vector fields in $\mathcal{V}^{-}(x)$ to avoid certain variationally critical portions that do not correspond to OGC's.

Clearly, we are interested in determining existence of geometrically critical values. In order to use a variational approach we will first have to keep into consideration the more general class of $\mathcal{V}^{+}$-variationally critical portions. A central issue in our theory consists in studying the relations between $\mathcal{V}^{+}$-variational critical portions $x_{\mid[a, b]}$ and OGC's. From now on $V^{+}$-variationally critical portions, will be simple called variationally critical portions. 


\section{ClASSIFICATION OF VARIATIONALly CRITICAL PORTIONS}

Let us now take a look at how variationally critical portions look like. We will interested to variationally critical portion $x_{\mid[a, b]}$ such that $[a, b] \in \mathcal{I}_{x}^{0}$.

In first place, let us show that smooth variationally critical points are OGC's; we need a preparatory result:

Lemma 4.1. Let $x \in \mathfrak{M}$ be fixed, and let $[a, b] \in \mathcal{I}_{x}^{0}$ be such that $x_{\mid[a, b]}$ is a (non-constant) variationally critical portion of $x$. Then:

(1) if $[\alpha, \beta] \subset[a, b]$ is such that $x(] \alpha, \beta[) \subset \Omega$, then $x_{\mid[\alpha, \beta]}$ is a geodesic;

(2) $x^{-1}(\partial \Omega) \cap[a, b]$ consists of a finite number of closed intervals and isolated points;

(3) $x$ is constant on each connected component of $x^{-1}(\partial \Omega) \cap[a, b]$;

(4) $x_{\mid[a, b]}$ is piecewise $C^{2}$, and the discontinuities of $\dot{x}$ may occur only at points in $\partial \Omega$

(5) each $C^{2}$ portion of $x_{\mid[a, b]}$ is a geodesic in $\bar{\Omega}$.

(6) $\inf \{\phi(x(s)): s \in[a, b]\}<-\delta_{0}$.

Proof. Let $[\alpha, \beta] \subset[a, b]$ be such that $x([\alpha, \beta]) \subset \Omega$. In this case the set of the restrictions to $[\alpha, \beta]$ of the vector fields in $\mathcal{V}^{+}(x)$ contains the Hilbert space $H_{0}^{1}\left([\alpha, \beta], \mathbb{R}^{N}\right)$. Variational criticality for $x$ implies then:

$$
\int_{\alpha}^{\beta} g\left(\dot{x}, \frac{\mathrm{D}}{\mathrm{d} s} V\right) \mathrm{d} s \geq 0, \quad \text { and } \int_{\alpha}^{\beta} g\left(\dot{x},-\frac{\mathrm{D}}{\mathrm{d} s} V\right) \mathrm{d} s \geq 0,
$$

i.e., $\int_{\alpha}^{\beta} g\left(\dot{x}, \frac{\mathrm{D}}{\mathrm{d} s} V\right) \mathrm{d} s=0$ for all $V \in H_{0}^{1}\left([\alpha, \beta], \mathbb{R}^{N}\right)$, which tells us that $x_{\mid[\alpha, \beta]}$ is a geodesic in $\Omega$. Similarly, if $[\alpha, \beta] \subset[a, b]$ is such that $x(] \alpha, \beta[) \subset \Omega$ and $x(\alpha), x(\beta) \in$ $\partial \Omega$, by a limit argument we get that $x_{\mid[\alpha, \beta]}$ is a geodesic proving (1). Now observe that the strong concavity assumption on $\bar{\Omega}$ implies (see $(1.7)$ ) that there exists $\bar{t} \in] \alpha, \beta$ [ such that $\phi(x(\bar{t}))<-\delta_{0}$. By Lemma 2.1. $\beta-\alpha \geq \frac{\delta_{0}^{2}}{K_{0}^{2}}\left(\int_{\alpha}^{\beta} g(\dot{x}, \dot{x}) \mathrm{d} t\right)^{-1}$ and hence the number of such intervals $[\alpha, \beta]$ must be finite, which proves part (2) of the thesis.

Now, let us prove that if $[\alpha, \beta] \subset[a, b]$ is such that $x([\alpha, \beta]) \subset \partial \Omega$, then $x$ is constant on $[\alpha, \beta]$.

The set of restrictions to $[\alpha, \beta]$ of vector fields in $\mathcal{V}^{+}(x)$ clearly contains (properly) the Hilbert subspace of $H_{0}^{1}\left([\alpha, \beta], \mathbb{R}^{N}\right)$ consisting of those $V$ such that $V(s) \in T_{x(s)} \partial \Omega$ for any $s \in[\alpha, \beta]$. Variational criticality in this case implies that

$$
\int_{\alpha}^{\beta} g\left(\dot{x}, \frac{\mathrm{D}}{\mathrm{d} s} V\right) \mathrm{d} s \geq 0 \quad \text { and } \quad \int_{\alpha}^{\beta} g\left(\dot{x},-\frac{\mathrm{D}}{\mathrm{d} s} V\right) \mathrm{d} s \geq 0
$$

for any $V$ such that $V(s) \in T_{x(s)}(\partial \Omega)$ for all $s \in[\alpha, \beta]$, and satisfying $V(\alpha)=V(\beta)=0$. Then $x_{\mid[\alpha, \beta]}$ is a geodesic in $\partial \Omega$ with respect to $g$, so there exists $\lambda \in \mathcal{C}^{0}([\alpha, \beta], \mathbb{R})$ such that

$$
\frac{\mathrm{D}}{\mathrm{d} s} \dot{x}(s)=\lambda(s) \nabla \phi(\gamma(s)), \quad \forall s \in[\alpha, \beta]
$$

Now consider the second derivative of $\phi(x(s)) \equiv 0$ in $[\alpha, \beta]$. We obtain

$$
\begin{aligned}
0=H^{\phi}(x(s))[\dot{x}(s), \dot{x}(s)] & +g\left(\frac{\mathrm{D}}{\mathrm{d} s} \dot{x}(s), \nabla \phi(x(s))\right)= \\
& =H^{\phi}(x(s))[\dot{x}(s), \dot{x}(s)]+\lambda(s) g(\nabla \phi(x(s)), \nabla \phi(x(s))),
\end{aligned}
$$

for all $s \in[\alpha, \beta]$. Suppose by contradiction that $x_{\mid[\alpha, \beta]}$ is not constant. Then, by strong concavity condition $H^{\phi}(x(s))[\dot{x}(s), \dot{x}(s)]<0$ so

$$
\lambda(s)>0, \quad \forall s \in[\alpha, \beta] .
$$


But, choosing

$$
V(s)= \begin{cases}\sin \left(\frac{s-\alpha}{\beta-\alpha} \pi\right) \nabla \phi(x(s)) & \text { if } s \in[\alpha, \beta] \\ 0 & \text { otherwise, }\end{cases}
$$

then $V \in \mathcal{V}^{+}(x)$ and

$$
\begin{aligned}
0 \leq \int_{\alpha}^{\beta} g\left(\dot{x}, \frac{\mathrm{D}}{\mathrm{d} s} V\right) \mathrm{d} s=- & \int_{\alpha}^{\beta} g\left(\frac{\mathrm{D}}{\mathrm{d} s} x, V\right) \mathrm{d} s= \\
& \quad-\int_{\alpha}^{\beta} \lambda(s) \sin \left(\frac{s-\alpha}{\beta-\alpha} \pi\right) g(\nabla \phi(x(s)), \nabla \phi(x(s))) \mathrm{d} s,
\end{aligned}
$$

in contradiction with 4.2. Then $x_{\mid[\alpha, \beta]}$ is constant and (3) is proven.

Since, as we have seen above, $x$ is a geodesic when it does not touch the boundary, (1), (2) (3) imply (4) and (5).

Finally, since $x_{\mid[a, b]}$ is not constant, it is not included in $\partial \Omega$, therefore by (1) and (1.7) we obtain (6).

We are now ready for the proof of the following:

Proposition 4.2. Assume that there is no WOGC in $\bar{\Omega}$. Let $x \in \mathfrak{M}$ and $[a, b] \in \mathcal{I}_{x}^{0}$ be such that:

- $x_{\mid[a, b]}$ is a variationally critical portion of $x$,

- the restriction of $x$ to $[a, b]$ is of class $C^{1}$.

Then, $x_{\mid[a, b]}$ is a orthogonal geodesic chord in $\bar{\Omega}$ (with $x(] a, b[) \subset \Omega$ ).

Proof. $C^{1}$-regularity, (2) and (3) of Lemma 4.1 shows that $x^{-1}(\partial \Omega) \cap[a, b]$ consists only of a finite number of isolated points. Then, by the $C^{1}$-regularity on $[a, b]$ and parts (4)(5) of Lemma 4.1, $x$ is a geodesic on the whole interval $[a, b]$. Moreover an integration by parts shows that $\dot{x}(a)$ and $\dot{x}(b)$ are orthogonal to $T_{x(a)} \partial \Omega$ and $T_{x(b)} \partial \Omega$ respectively. Finally, since there are not any WOGC on $\bar{\Omega}, x_{\mid[a, b]}$ is an OGC.

Variationally critical portions $x_{\mid[a, b]}$ of class $C^{1}$ will be called regular variationally critical portions; those critical portions that do not belong to this class will be called irregular. Irregular variationally critical portions of curves $x \in \mathfrak{M}$ are further divided into two subclasses, described below.

Assume that there are not WOGC's in $\bar{\Omega}$.

Proposition 4.3. Let $x \in \mathfrak{M}$ and let $[a, b] \in \mathcal{I}_{x}^{0}$ be such that $x_{\mid[a, b]}$ is an irregular variationally critical portion of $x$.

Then, there exists a subinterval $[\alpha, \beta] \subset[a, b]$ such that $x_{\mid[a, \alpha]}$ and $x_{\mid[\beta, b]}$ are constant (in $\partial \Omega$ ), $\dot{x}\left(\alpha^{+}\right) \in T_{x(\alpha)}(\partial \Omega)^{\perp}, \dot{x}\left(\beta^{-}\right) \in T_{x(\beta)}(\partial \Omega)^{\perp}$, and one of the two mutually exclusive situations occur:

(1) there exists a finite number of intervals $\left.\left[t_{1}, t_{2}\right] \subset\right] \alpha, \beta\left[\right.$ such that $x\left(\left[t_{1}, t_{2}\right]\right) \subset \partial \Omega$ and that are maximal with respect to this property; moreover, $x$ is constant on each such interval $\left[t_{1}, t_{2}\right]$, and $\dot{x}\left(t_{1}^{-}\right) \neq \dot{x}\left(t_{2}^{+}\right)$; $]$

(2) $x_{\mid[\alpha, \beta]}$ is an $O G C$ in $\bar{\Omega}$.

(Note that in the above statement it may happen $\alpha=a$ or $\beta=b$.)

Proof. Totally analogous to the proof of Lemma 4.1 and Proposition 4.2.

Irregular variationally critical portions in the class described in part (1) will be called of first type, those described in part (2) will be called of second type. An interval $\left[t_{1}, t_{2}\right]$ as in part (1) will be called a cusp interval of the irregular variational critical portion $x_{\mid[a, b]}$ (see Figure 3) also in the degenerate case $t_{1}=t_{2}$.

\footnotetext{
${ }^{3}$ When $t_{1}<t_{2}$ an easy partial integration argument shows that both $\dot{x}\left(t_{1}^{-}\right)$and $\dot{x}\left(t_{2}^{+}\right)$are orthogonal to $\partial \Omega$.
} 


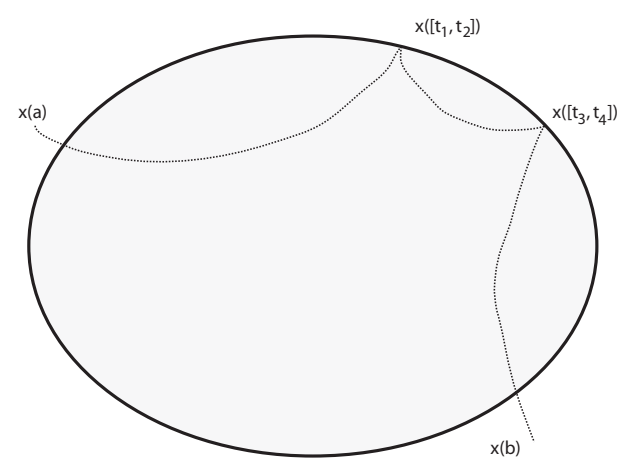

FIGURE 3. Irregular critical portions of curves in $\mathfrak{M}$, with cusp intervals $\left[t_{1}, t_{2}\right]$ and $\left[t_{3}, t_{4}\right]$.

Remark 4.4. We observe here that, due to the strict concavity assumption, if $x_{\mid[a, b]}$ is an irregular variationally critical portion of first type and $\left[t_{1}, t_{2}\right],\left[s_{1}, s_{2}\right]$ are cusp intervals for $x$ contained in $[a, b]$ with $t_{2}<s_{1}$, then

$$
\text { there exists } \left.s_{0} \in\right] t_{2}, s_{1}\left[\text { with } \phi\left(x\left(s_{0}\right)\right)<-\delta_{0},\right.
$$

(see (1.7)). This implies that the number of cusp intervals of irregular variationally critical portions $x_{\mid[a, b]}$ is uniformly bounded (cf. Lemma 2.1 and recall that $x \in \mathfrak{M}$ and $[a, b] \in \mathcal{I}_{x}$ implies $\left.\frac{1}{2} \int_{a}^{b} g(\dot{x}, \dot{x}) \mathrm{d} t<M_{0}\right)$.

If $x_{[[a, b]}$ is a irregular critical portion of the first type, and if $\left[t_{1}, t_{2}\right]$ is a cusp interval of $x_{\mid[a, b]}$, we will set

$$
\Theta_{x}\left(t_{1}, t_{2}\right)=\text { the (non oriented) angle between the vectors } \dot{x}\left(t_{1}^{-}\right) \text {and } \dot{x}\left(t_{2}^{+}\right) .
$$

Observe that $\left.\left.\Theta_{x}\left(t_{1}, t_{2}\right) \in\right] 0, \pi\right]$, (since $\left.x([a, b]) \subset \bar{\Omega}\right)$.

Remark 4.5. We observe that if $\left[t_{1}, t_{2}\right] \subset[a, b] \in \mathcal{I}_{x}^{0}$ is a cusp interval of $x_{\mid[a, b]}$, then the tangential components of $\dot{x}\left(t_{1}^{-}\right)$and of $\dot{x}\left(t_{2}^{+}\right)$along $\partial \Omega$ are equal; this is is easily obtained by an integration by parts. It follows that if $\Theta_{x}\left(t_{1}, t_{2}\right)>0$, then $\dot{x}\left(t_{1}^{-}\right)$and $\dot{x}\left(t_{2}^{+}\right)$cannot be both tangent to $\partial \Omega$.

We will denote by $\mathcal{Z}$ the set of all curves having variationally critical portions:

$$
\mathcal{Z}=\left\{x \in \mathfrak{M}: \exists[a, b] \subset[0,1] \text { such that } x_{\mid[a, b]} \text { is a variationally critical portion of } x\right\} .
$$

The following compactness property holds for $\mathcal{Z}$ :

Proposition 4.6. Let $x_{n}$ be a sequence in $\mathcal{Z},\left[a_{n}, b_{n}\right] \in \mathcal{I}_{x_{n}}^{0}$ for any $n$ such that $x_{n \mid\left[a_{n}, b_{n}\right]}$ is a (non constant) variationally critical portion of $x_{n}$. Then, up to subsequences, $a_{n}$ converges to some $a, b_{n}$ converges to some $b$, with $0 \leq a<b \leq 1$, and the sequence $x_{n}$ : $\left[a_{n}, b_{n}\right] \rightarrow \bar{\Omega}$ is $H^{1}$-convergent (in the sense of Remark 1.8 ) to some curve $x:[a, b] \rightarrow \bar{\Omega}$ as $n \rightarrow \infty,[a, b] \in \mathcal{I}_{x}^{0}$, and $x_{\mid[a, b]}$ is a variationally critical portion of $x$.

Proof. By Lemma 2.1 and (1.7), the sequence $b_{n}-a_{n}$ is bounded away from 0, which implies the existence of subsequences converging in $[0,1]$ to $a$ and $b$ respectively, and with $a<b$. If $x_{n}$ is a sequence of regular variationally critical portions, then the conclusion follows easily observing that $x_{n}$, and thus $\widehat{x}_{n}$ (its affine reparameterization in $[a, b]$ ) is a sequence of geodesics with image in a compact set and having bounded energy.

For the general case, one simply observes that the number of cusp intervals of each $x_{n}$ is bounded uniformly in $n$, and the argument above can be repeated by considering the restrictions of $x_{n}$ to the complement of the union of all cusp intervals. Finally, integrating by parts the term $\int_{a}^{b} g\left(\dot{x}, \frac{\mathrm{D}}{\mathrm{d} t} V\right) \mathrm{d} t$ one observes that it is non negative for all $V \in \mathcal{V}^{+}(x)$, 
hence $x_{\mid[a, b]}$ is a variationally critical portion of $x$ (note that the uniform convergency implies $\left.[a, b] \in \mathcal{I}_{x}^{0}\right)$.

Remark 4.7. We point out that the first part of the proof of Proposition 4.6 shows that if $x_{n} \in \mathcal{Z}(\subset \mathfrak{M}),\left[a_{n}, b_{n}\right] \in \mathcal{I}_{x_{n}}^{0}$ and $x_{n \mid\left[a_{n}, b_{n}\right]}$ is an OGC, then, up to subsequences, there exists $[a, b] \subset[0,1]$ and $x:[a, b] \rightarrow \bar{\Omega}$ such that $x_{n \mid\left[a_{n}, b_{n}\right]} \rightarrow x_{\mid[a, b]}$ in $H^{1}$ and $x_{\mid[a, b]}$ is an OGC.

Since we are assuming that there are not any WOGC in $\bar{\Omega}$ by Lemma 4.1 , Proposition 4.2. Proposition 4.3 and Proposition 4.6, we immediately obtain the following result.

Corollary 4.8. There exists $d_{0}>0$ such that for any $x_{\mid[a, b]}$ irregular variationally critical portion of first type with $[a, b] \in \mathcal{I}_{x}^{0}$, there exists a cusp interval $\left[t_{1}, t_{2}\right] \subset[a, b]$ for $x$ such that

$$
\Theta_{x}\left(t_{1}, t_{2}\right) \geq d_{0}
$$

The above corollary states that every irregular variational critical portion of the first type has at least a cusp with "amplitude" greater than or equal to a positive constant $d_{0}$.

Remark 4.9. For any $\left.\delta \in] 0, \delta_{0}\right]$ we have the following property: for any $x \in \mathfrak{M}$ and $[a, b] \in \mathcal{I}_{x}^{0}$ such that $x_{\mid[a, b]}$ is an irregular variationally critical portion of first type, there exists an interval $[\alpha, \beta] \subset[a, b]$ and a cusp interval $\left[t_{1}, t_{2}\right] \subset[\alpha, \beta]$ such that:

$$
\Theta_{x}\left(t_{1}, t_{2}\right) \geq d_{0}, \text { and } \phi(x(\alpha))=\phi(x(\beta))=-\delta,
$$

where $d_{0}$ is given in Corollary 4.8 .

Note that $g(\nabla \phi(x(\alpha)), \dot{x}(\alpha))>0$ and $g(\nabla \phi(x(\beta)), \dot{x}(\beta))<0$ by the strong concavity assumption.

\section{SOME RESUlts about CRITICAL PORTIONS With RESPECT TO $\mathcal{V}^{-}$}

In this section we shall discuss some results concerning the notion of $\mathcal{V}^{-}$-variational critical portions. It is important to point out that, to avoid curves having irregular variational critical portions of first type, we will use integral flows for fields in the cone $\mathcal{V}^{-}(x)$ defined in (3.3). This will be possible thanks to the regularity property described in Lemma 5.1 below.

Moreover thanks to the assumption of strong concavity we shall obtain also Lemma 5.8 that may have an important role to study the multiplicity problem.

Let us denote by $\mathcal{A}$ an arbitrary open subset of $M$ with regular boundary, and let $\phi_{\mathcal{A}}: M \rightarrow \mathbb{R}$ be a $C^{2}$-function such that $\phi_{\mathcal{A}}(q)<0$ iff $q \in \mathcal{A}, \phi_{\mathcal{A} \mid \partial \mathcal{A}}=0$ and $\nabla \phi_{\mathcal{A}} \neq 0$ on $\partial \mathcal{A}$. (Note that in Remark 1.2, $\phi=\phi_{\Omega}$ ). Set

(5.1) $\mathcal{V}_{\mathcal{A}}^{-}(x)=\left\{V \in T_{x} H^{1}\left([0,1], \mathbb{R}^{N}\right): g\left(V(s), \nabla \phi_{\mathcal{A}}(x(s))\right) \leq 0\right.$ for $\left.x(s) \in \partial \mathcal{A}\right\}$.

Lemma 5.1. Let $y \in H^{1}([a, b], \overline{\mathcal{A}})$ be such that:

$$
\int_{a}^{b} g\left(\dot{y}, \frac{\mathrm{D}}{\mathrm{d} t} V\right) \mathrm{d} t \geq 0, \quad \forall V \in \mathcal{V}_{\mathcal{A}}^{-}(y) \text { and } V(a)=V(b)=0 .
$$

Then, $y \in H^{2, \infty}([a, b], \overline{\mathcal{A}})$ and in particular it is of class $C^{1}$.

Proof. We refer the reader e.g. to [5, Lemma 3.2] for the proof.

Definition 5.2. If $y_{\mid[a, b]}$ satisfies 5.2 , it will be called $a \mathcal{V}_{\mathcal{A}}^{-}$-variationally critical portion.

Remark 5.3. It is important to observe that, while a variationally critical portion with respect to $\mathcal{V}^{-}$is of class $C^{1}$, this is not the case for variationally critical portion with respect to $\mathcal{V}^{+}$. In particular, as pointed out in Proposition 4.3 , irregular variationally critical portions of first type are not of class $C^{1}$.

Now, denote by $\nu(x)=\frac{\nabla \phi_{\mathcal{A}}(x)}{\left\|\nabla \phi_{\mathcal{A}}(x)\right\|}$ the outward pointing unit normal vector field in $\partial \mathcal{A}$. 
Lemma 5.4. Let $y \in H^{1}([a, b], \overline{\mathcal{A}})$ satisfying $(5.2)$. Then $g(\dot{y}, \dot{y})$ is constant on $[a, b]$, and (5.3)

$$
-\frac{\mathrm{D}}{\mathrm{d} s} \dot{y}(s)=\mu(s) \nu(y(s)) \text { a.e., } \quad \text { where } \mu(s)= \begin{cases}0, & \text { if } \phi_{\mathcal{A}}(y(s))<0, \\ \frac{H^{\phi} \mathcal{A}(y)[\dot{y}, \dot{y}]}{g\left(\nu(y), \nabla \phi_{\mathcal{A}}(y)\right)}, & \text { if } \phi_{\mathcal{A}}(y(s)=0 .\end{cases}
$$

Moreover

$$
\mu(y(s)) \leq 0 \text { a.e. }
$$

Proof. If $y_{\mid[a, b]}$ satisfies (5.2), by Lemma 5.1, $\frac{\mathrm{D}}{\mathrm{d} t} \dot{y} \in L^{\infty}$, so we can integrate by parts in (5.2) obtaining

$$
\int_{a}^{b}-g\left(\frac{\mathrm{D}}{\mathrm{d} t} \dot{y}, V\right) \mathrm{d} t \geq 0, \quad \text { for all } V \in C_{0}^{\infty}\left([a, b], \mathbb{R}^{N}\right)
$$

satisfying

$$
g(V(s), \nu(y(s))) \leq 0 \text { for any } s \text { such that } \phi_{\mathcal{A}}(y(s))=0 .
$$

Then $y$ is a geodesic where it does not intersect the boundary. Moreover using vector fields $V$ such that $g(V(s), \nu(y(s)))=0$ for all $s$ such that $\phi_{\mathcal{A}}(y(s))=0$ we obtain

$$
-\frac{\mathrm{D}}{\mathrm{d} s} \dot{y}(s)=\mu(s) \nu(y(s)) \text { a.e., } \quad \text { for some } \mu:[a, b] \rightarrow \mathbb{R} .
$$

Since $\frac{\mathrm{D}}{\mathrm{d} t} \dot{y}$ is in $L^{\infty}$, then $\mu \in L^{\infty}([0,1], \mathbb{R})$ (and $\mu(s)=0$ if $\phi_{\mathcal{A}}(y(s))<0$ ).

Now $y$ is of class $C^{1}$ and $\phi_{\mathcal{A}}(y(s)) \leq 0 \forall s \in[a, b]$. Therefore

$$
g\left(\nabla \phi_{\mathcal{A}}(y(s)), \dot{y}(s)\right)=0, \quad \text { for every } s \text { such that } \phi_{\mathcal{A}}(y(s))=0,
$$

and, contracting both terms of (5.5) with $\dot{y}$, we have

$$
g\left(\frac{\mathrm{D}}{\mathrm{d} t} \dot{y}, \dot{y}\right)=0 \quad \text { a.e. in }[a, b] .
$$

Then there exists $c_{1}>0$ such that

$$
g(\dot{y}, \dot{y})=c_{1} \quad \text { a.e. in }[a, b] .
$$

But $y$ is of $\operatorname{class} \mathcal{C}^{1}$ so

$$
g(\dot{y}, \dot{y})=c_{1}, \quad \forall s \in[a, b],
$$

Now contracting both members in (5.5) with $\nabla \phi_{\mathcal{A}}(y(s))$ we obtain

$$
-g\left(\frac{\mathrm{D}}{\mathrm{d} s} \dot{y}, \nabla \phi_{\mathcal{A}}(y)\right)=\mu(s) g\left(\nu(y), \nabla \phi_{\mathcal{A}}(y)\right), \quad \text { a.e. in }[a, b] .
$$

Set $C_{y}=\left\{s: \phi_{\mathcal{A}}(y(s))=0\right\}$ which is the set where $\mu(s)$ can be nonzero. Using well known theorems in Sobolev spaces (see [13]), by (5.6) we have (differentiating in $C_{y}$ )

$$
H^{\phi_{\mathcal{A}}}(y)[\dot{y}, \dot{y}]+g\left(\nabla \phi_{\mathcal{A}}(y), \frac{\mathrm{D}}{\mathrm{d} t} \dot{y}\right)=0 \quad \text { a.e. in } C_{y} .
$$

Then, by (5.7)

$$
\mu(s) g\left(\nu(y), \nabla \phi_{\mathcal{A}}(y)\right)=H^{\phi_{\mathcal{A}}}(y)[\dot{y}, \dot{y}] \quad \text { a.e. in } C_{y}
$$

from which we deduce (5.3).

Finally to prove (5.4) it is sufficient to apply formula (5.2) with

$$
V(s)=-\sin \left(\frac{s-a}{b-a} \pi\right) \nabla \phi_{\mathcal{A}}(y(s)),
$$

since $y$ satisfies (5.3).

Remark 5.5. Note that, under the assumption of strong concavity the set

$$
C_{y}=\{s \in[a, b]: \phi(y(s))=0\}
$$

consists of a finite number of intervals. On each one of these, $y$ is of class $C^{2}$, and it satisfies the "constrained geodesic" differential equation

$$
\frac{\mathrm{D}}{\mathrm{d} s} \dot{y}(s)=-\left[\frac{1}{g(\nu(y(s)), \nabla \phi(y(s)))} H^{\phi}(y(s))[\dot{y}(s), \dot{y}(s)] \nu(y(s)) .\right.
$$


Remark 5.6. Now suppose that $y$ satisfies $(5.2)$ and $\overline{\mathcal{A}}$ is convex. In this case

$$
H^{\phi_{\mathcal{A}}}(y)[\dot{y}, \dot{y}] \geq 0 \text { for any } s \text { such that } \phi_{\mathcal{A}}(y(s))=0 .
$$

Then from formulas (5.2) and 5.3) we see that the function $\mu$ in $(5.3)$ is identically zero, and thus any $\mathcal{V}_{\mathcal{A}}^{-}$-variationally critical portion in $\overline{\mathcal{A}}$ is a free geodesic in $\overline{\mathcal{A}}$.

Our main goal is to construct a functional for our minimax argument and a family of flows in the path space on which the energy functional is non increasing. Moreover, to clarify another trait of the strong concavity condition, we wish to point out the following property that may be useful also in view of obtaining multiplicity results: for any curve $x \in \mathfrak{M}$ and for any interval $[a, b] \in \mathcal{I}_{x}$ where $x$ is uniformly close to $\partial \Omega$, the curve $x_{\mid[a, b]}$ can be deformed in directions where $\phi$ increases, without increasing the energy functional. Such a property will be obtained thanks above all to Definition 5.7 and Lemma 5.8 below.

Definition 5.7. Fix $\left.\delta \in] 0, \delta_{0}\right]$. We say that $[\alpha, \beta] \subset[0,1]$ is a $\delta$-interval (for $x \in \mathfrak{M}$ ) if

$$
\begin{array}{r}
\phi(x(s) \geq-\delta \text { for all } s \in[\alpha, \beta], \phi(x(\alpha))=\phi(x(\beta))=0, \\
\text { and } \inf \{\phi(x(s)): s \in[\alpha, \beta]\}=-\delta .
\end{array}
$$

We say that a $\delta$-interval $[\alpha, \beta]$ is minimal if it is minimal with respect to the property above.

Lemma 5.8. For any $\left.\delta \in] 0, \delta_{0}\right]$, for any $x \in \mathfrak{M}$, for any $[\alpha, \beta] \delta$-interval for $x$, there exists $V \in H_{0}^{1}\left([\alpha, \beta], \mathbb{R}^{N}\right)$ such that

$$
g(\nabla \phi(x(s)), V(s)) \geq 0 \forall s \in[\alpha, \beta] \text { satisfying } \phi(x(s))=-\delta \text { and } \phi(x(s))=0 ;
$$

and

$$
\int_{\alpha}^{\beta} g\left(\dot{x}, \frac{\mathrm{D}}{\mathrm{d} s} V\right) \mathrm{d} s<0 .
$$

Proof. Clearly we can assume that the $\delta$-interval $[\alpha, \beta]$ is minimal with respect to (5.9). Suppose by contradiction that for any $\left.V \in H_{0}^{1}\left([\alpha, \beta], \mathbb{R}^{N}\right), 5.10\right)$ and (5.11) are not satisfied at the same time. Then by the minimimality property of $[\alpha, \beta]$ we see that

$$
\int_{\alpha}^{\beta} g\left(\dot{x}, \frac{\mathrm{D}}{\mathrm{d} s} V\right), \mathrm{d} s \geq 0
$$

for any $V \in H_{0}^{1}\left([\alpha, \beta], \mathbb{R}^{N}\right)$ satisfying $g(\nabla \phi(x(s)), V(s)) \geq 0$ for any $s \in[\alpha, \beta]$ such that $\phi(x(s))=-\delta$.

Then the curve $x_{\mid[\alpha, \beta]}$ is a $\mathcal{V}_{\mathcal{A}}^{-}$-variationally critical portion in $\overline{\mathcal{A}}=\phi^{-1}([-\delta,+\infty[)$. Since $\left.\delta \in] 0, \delta_{0}\right]$, by Remark $1.4 \overline{\mathcal{A}}$ is convex. Then from Remark $5.6 x_{\mid[\alpha, \beta]}$ is a free geodesic. Since it has endpoints on $\phi^{-1}(0), 0<\delta \leq \delta_{0}$ and $\inf _{[\alpha, \beta]} \phi(x(s))=-\delta$, we have a contradiction with the strong concavity assumption on $\bar{\Omega}$ (cf. Remark 1.4). This concludes the proof.

For the Palais-Smale condition described in Proposition 6.1 below will be useful also the following notion.

Definition 5.9. Fix $\left.\delta \in] 0, \delta_{0}\right]$ and $\mathfrak{d} \in[0, \delta[$. We say that $[\alpha, \beta] \subset[0,1]$ is a $(\delta, \mathfrak{d})$-interval (for $x \in \mathfrak{M}$ ) if

$$
\begin{array}{r}
\phi(x(s) \geq-\delta \forall s \in[\alpha, \beta], \phi(x(\alpha))=\phi(x(\beta))=-\mathfrak{d}, \\
\text { and } \inf \{\phi(x(s)): s \in[\alpha, \beta]\}=-\delta .
\end{array}
$$




\section{The PALAIS-SMALE CONDITION FOR $\mathcal{V}^{+}$-VARIATIONALLY CRITICAL PORTION}

Let $\delta_{0}$ be as in Remark 1.4. We will now proceed with the statement and the proof of the analogue of the classical Palais-Smale condition which is suited to our variational framework; unfortunately, the technical nature of the following Propositions cannot be avoided. Their statements contains several different facts that should be familiar to specialists in pseudo-gradient techniques. Recall that when proving the Palais-Smale condition for $C^{1}$ functionals on Banach manifolds, one first needs to show that at each non critical point there must exist a direction along which the functional decreases. Secondly, one needs to show that the descent direction for the functional at a given point can be chosen in such a way to be a decreasing direction also in a neighborhood of this point; along such direction the decreasing rate of the functional should be bounded away from zero uniformly outside a neighborhood of the set of critical points. In our context, a further complication is given by the fact that our notion of criticality is defined for portions of the curve $x$, so that, to single out a decreasing direction at each non critical path, we need the possibility to patch together vector fields defined on each non critical portion of $x$. All these facts are condensed into Propositions 6.1 and 6.4 below; for a better understanding of the statement, it may be useful to keep in mind the following description of the quantities involved in the hypotheses and the theses:

- the positive number $r$ is a measure of distance from variationally critical portions;

- $\theta(r)$ is the rate of increase of the function $\phi$ along the flow at those points of the curve that are in $\phi^{-1}([-\kappa(r), \kappa(r)])$; and also at that points in $\phi^{-1}(-\delta)$ and $\phi^{-1}(-\mathfrak{d})$ corresponding to instants included in $(\delta, \mathfrak{d})$-intervals with $\left.\left.\delta \in\right] \kappa(r), \delta_{0}\right]$ and $\mathfrak{d} \in[0, \kappa(r)]$ (cf. (1) in the statement of Proposition 6.1).

- $\mu(r)$ is the rate of decrease of the functional $\mathcal{F}$, that will be defined formally in Section 8 .

For $[a, b] \subset[0,1]$, we denote by $\mathcal{Z}_{a, b}$ the set of curves $w:[a, b] \rightarrow \bar{\Omega}$ that are variationally critical portions:

$$
\begin{aligned}
& \mathcal{Z}_{a, b}=\left\{w \in H^{1}([a, b], \bar{\Omega}): w(a), w(b) \in \partial \Omega\right. \\
& \left.\qquad \int_{a}^{b} g\left(\dot{w}, \frac{\mathrm{D}}{\mathrm{d} t} V\right) \mathrm{d} t \geq 0 \text { for all } V \in \mathcal{V}^{+}(w)\right\},
\end{aligned}
$$

where $\mathcal{V}^{+}$is defined in (3.1).

We shall need the following results:

Proposition 6.1. For all $r>0$, there exist positive numbers $\theta(r), \mu(r), \kappa(r)\left(\kappa(r)<\delta_{0}\right)$ satisfying the following property: for all $x \in \mathfrak{M}_{0}$ and for all $[a, b] \subset[0,1]$ for which

$$
x(a), x(b) \in \partial \Omega \text { and } x([a, b]) \subset \bar{\Omega}
$$

(namely $[a, b] \in \mathcal{I}_{x}^{0}$ ),

$$
\frac{1}{4}\left(\frac{3 \delta_{0}}{4 K_{0}}\right)^{2} \leq \frac{b-a}{2} \int_{a}^{b} g(\dot{x}, \dot{x}) \mathrm{d} s, \quad \frac{1}{2} \int_{a}^{b} g(\dot{x}, \dot{x}) \mathrm{d} s \leq M_{0},
$$

and such that

$$
\left\|x_{\mid[a, b]}-y\right\|_{a, b} \geq r, \quad \forall y \in \mathcal{Z}_{a, b},
$$

there exists a vector field $V_{x} \in H^{1}\left([a, b], \mathbb{R}^{N}\right)$ such that the following conditions hold:

(1) for any $\left.\delta \in] \kappa(r), \delta_{0}\right]$, for any $\mathfrak{d} \in[0, \kappa(r)]$, for any $(\delta, \mathfrak{d})$-interval $[c, d] \subset$ $[a, b]$, and for all $s \in[c, d]$ such that $\phi(x(s)=-\delta$ or $\phi(x(s)=-\mathfrak{d}$, it is $g\left(\nabla \phi(x(s)), V_{x}(s)\right) \geq \theta(r)\left\|V_{x}\right\|_{a, b}$; 
(2) $g\left(\nabla \phi(x(s)), V_{x}(s)\right) \geq \theta(r)\left\|V_{x}\right\|_{a, b}$ for all $s \in[a, b]$ that satisfies $\phi(x(s)) \in$ $[-\kappa(r), \kappa(r)]$;

(3) $\int_{a}^{b} g\left(\dot{x}, \frac{\mathrm{D}}{\mathrm{d} s} V_{x}\right) \mathrm{d} s \leq-\mu(r)\left\|V_{x}\right\|_{a, b}$.

Remark 6.2. About assumption (6.3) we wish to point out that we shall look for OGC's with energy integral $\geq \frac{1}{2}\left(\frac{3 \delta_{0}}{4 K_{0}}\right)^{2}$. On the other hand if $\frac{b-a}{2} \int_{a}^{b} g(\dot{x}, \dot{x}) \mathrm{d} s \leq \frac{1}{2}\left(\frac{3 \delta_{0}}{4 K_{0}}\right)^{2}$ and $x(a) \in \partial \Omega$, by Lemma 2.1 one deduce that $x([a, b]) \subset\left\{\phi \geq-\frac{3 \delta_{0}}{4}\right\}$ so, if also $x(b) \in \partial \Omega$ $x_{\mid[a, b]}$ can not be a geodesic because strong concavity assumption.

Proof of Proposition 6.1. We argue by contradiction, assuming the existence of sequences $\theta_{n} \rightarrow 0^{+}, \mu_{n} \rightarrow 0^{+}, \kappa_{n} \rightarrow 0^{+}, x_{n} \in \mathfrak{M}_{0},\left[a_{n}, b_{n}\right] \subset[0,1]$, satisfying

$$
\left.x_{n}\left(a_{n}\right), x_{n}\left(b_{n}\right) \in \partial \Omega \text { and } x_{n}\left(\left[a_{n}, b_{n}\right]\right) \subset \bar{\Omega}, \quad \text { (i.e., }\left[a_{n}, b_{n}\right] \in \mathcal{I}_{x_{n}}^{0}\right)
$$

and

$$
\frac{1}{4}\left(\frac{3 \delta_{0}}{4 K_{0}}\right)^{2} \leq \frac{b_{n}-a_{n}}{2} \int_{a_{n}}^{b_{n}} g\left(\dot{x}_{n}, \dot{x}_{n}\right) \mathrm{d} s, \quad \frac{1}{2} \int_{a_{n}}^{b_{n}} g\left(\dot{x}_{n}, \dot{x}_{n}\right) \mathrm{d} s \leq M_{0}
$$

and such that, for any $V \in H^{1}\left(\left[a_{n}, b_{n}\right], \mathbb{R}^{N}\right)$, the following properties cannot hold at the same time:

(C1) for any $\left.\delta \in] \kappa_{n}, \delta_{0}\right]$, for any $\mathfrak{d} \in\left[0, \kappa_{n}\right]$, for any $(\delta, \mathfrak{d})$-interval $[c, d] \subset\left[a_{n}, b_{n}\right]$, for all $s \in[c, d]$ such that $\phi\left(x_{n}(s)\right)=-\delta$ or $\phi\left(x_{n}(s)\right)=-\mathfrak{d}$, it is

$$
g\left(\nabla \phi\left(x_{n}(s)\right), V(s)\right) \geq \theta_{n}\|V\|_{a_{n}, b_{n}}
$$

(C2) $g\left(\nabla \phi\left(x_{n}(s)\right), V(s)\right) \geq \theta_{n}\|V\|_{a_{n}, b_{n}}$ for all $s \in\left[a_{n}, b_{n}\right]$ that satisfies $\phi\left(x_{n}(s)\right) \in$ $\left[-\kappa_{n}, \kappa_{n}\right]$

(C3) $\int_{a_{n}}^{b_{n}} g\left(\dot{x}_{n}, \frac{\mathrm{D}}{\mathrm{d} s} V\right) \mathrm{d} s \leq-\mu_{n}\|V\|_{a_{n}, b_{n}}$.

Note that, by (6.6),

$$
b_{n}-a_{n} \geq \frac{1}{4 M_{0}}\left(\frac{3 \delta_{0}}{4 K_{0}}\right)^{2} .
$$

Then, up to subsequences, we can assume the existence of the limits

$$
0 \leq \widehat{a}=\lim _{n \rightarrow \infty} a_{n}<\widehat{b}=\lim _{n \rightarrow \infty} b_{n} \leq 1,
$$

and up to affine reparameterizations we can assume $a_{n}=\widehat{a}, b_{n}=\widehat{b}$ for all $n$.

Moreover, up to subsequences again, we can assume that the reparameterization $x_{n \mid[\widehat{a}, \widehat{b}]}$ is weakly $H^{1}$-convergent (and therefore uniformly) to some curve $x \in H^{1}\left([\widehat{a}, \widehat{b}], \mathbb{R}^{N}\right)$, because $x_{n}$ satisfies (6.6) and $x_{n}(\widehat{a}) \in \partial \Omega$ is bounded.

To carry out the proof we shall show that

$$
x_{\mid[\widehat{a}, \widehat{b}]} \in \mathcal{Z}_{\widehat{a}, \widehat{b}},
$$

and

$$
x_{n \mid[\widehat{a}, \widehat{b}]} \rightarrow x_{\mid[\widehat{a}, \widehat{b}]} \text { strongly in } H^{1},
$$

obtaining a contradiction with (6.7).

To prove (6.8), suppose that there exists $\left.\delta \in] 0, \delta_{0}\right]$, and a minimal $\delta$-interval for $x$, $[c, d] \subset[\widehat{a}, \widehat{b}]$. Take $V \in \mathcal{V}^{+}(x)$ such that $V=0$ outside $[c, d]$ and such that

$$
g(\nabla \phi(x(s)), V(s)) \geq 0 \text { for all } s \in[c, d]: \phi(x(s))=-\delta .
$$

Thanks to the minimality property of $[c, d]$, we can take $V_{n}=V+\lambda_{n} \nabla \phi\left(x_{n}\right)$ with $\lambda_{n} \rightarrow 0^{+}$and such that $V_{n}$ satisfies (C1) and (C2) above with $V$ replaced by $V_{n}$. This can 
be proved since $x_{n \mid[\widehat{a}, \widehat{b}]}$ is included in $\bar{\Omega}$, it is bounded in $H^{1}, x_{n}$ uniformly converges to $x, \theta_{n}$ and $\kappa_{n}$ are infinitesimals, and $\nabla \phi$ does not vanish on $\phi^{-1}\left(\left[-\delta_{0}, \delta_{0}\right]\right)$.

Then property (C3) cannot hold, so

$$
\int_{\widehat{a}}^{\widehat{b}} g\left(\dot{x}_{n}, \frac{\mathrm{D}}{\mathrm{d} s} V_{n}\right) \mathrm{d} s>-\mu_{n}\left\|V_{n}\right\|_{\widehat{a}, \widehat{b}} .
$$

Since $\left\|V_{n}\right\|_{\widehat{a}, \widehat{b}}$ is bounded, 6.11) gives

$$
\liminf _{n \rightarrow \infty} \int_{\widehat{a}}^{\widehat{b}} g\left(\dot{x}_{n}, \frac{\mathrm{D}}{\mathrm{d} s} V_{n}\right) \mathrm{d} s \geq 0 .
$$

Since $\lambda_{n} \rightarrow 0$ and $x_{n}$ is bounded in $H^{1}, V_{n}$ is strongly $H^{1}$-convergent to $V$. Moreover $\dot{x}_{n}$ is weakly $L^{2}$-convergent to $\dot{x}$ on $[\widehat{a}, \widehat{b}]$, so we have

$$
0 \leq \lim _{n \rightarrow \infty} \int_{\widehat{a}}^{\widehat{b}} g\left(\dot{x}_{n}, \frac{\mathrm{D}}{\mathrm{d} s} V_{n}\right) \mathrm{d} s=\int_{\widehat{a}}^{\widehat{b}} g\left(\dot{x}, \frac{\mathrm{D}}{\mathrm{d} s} V\right) \mathrm{d} s=\int_{c}^{d} g\left(\dot{x}, \frac{\mathrm{D}}{\mathrm{d} s} V\right) \mathrm{d} s,
$$

because $V=0$ outside $[c, d]$. Since $V \in \mathcal{V}^{+}(x)$ satisfying (6.10) is arbitrary, by Lemma 5.8, we obtain a contradiction. Then there are not $\delta$-intervals for $x$ in $[\widehat{a}, \widehat{b}]$ and we can therefore choose any $V \in \mathcal{V}^{+}(x)$ to construct $V_{n}$ as above proving that $x_{\mid[\widehat{a}, \widehat{b}]}$ satisfies (6.12) for any $V \in \mathcal{V}^{+}(x)$. Then $x_{\mid[\widehat{a}, \widehat{b}]}$ satisfies (6.8).

To prove (6.9), let us choose a vector field $V_{n}$ along $x_{n \mid[\widehat{a}, \widehat{b}]}$ of the form

$$
V_{n}=-\gamma_{n}+\lambda_{n} \nabla \phi\left(x_{n}\right),
$$

where $\gamma_{n}=x_{n}-x$, and $\lambda_{n}$ can be chosen such that $\lambda_{n} \rightarrow 0^{+}$and $V_{n}$ satisfies properties (C1) and (C2) above with $V$ replaced by $V_{n}$. Then property (C3) cannot hold. Therefore it must be

$$
\liminf _{n \rightarrow \infty} \int_{\widehat{a}}^{\widehat{b}} g\left(\dot{x}_{n}, \frac{\mathrm{D}}{\mathrm{d} s} V_{n}\right) \mathrm{d} s \geq 0 .
$$

Since $\lambda_{n} \nabla \phi\left(x_{n}\right)$ is $H^{1}$-convergent to 0 , and $V_{n}=-\gamma_{n}+\lambda_{n} \nabla \phi\left(x_{n}\right)$,

$$
\liminf _{n \rightarrow \infty} \int_{\widehat{a}}^{\widehat{b}}-g\left(\dot{x}_{n}, \dot{\gamma}_{n}\right) \mathrm{d} s \geq 0
$$

that is

$$
\limsup _{n \rightarrow \infty} \int_{\widehat{a}}^{\widehat{b}} g\left(\dot{x}_{n}, \dot{x}_{n}-\dot{x}\right) \mathrm{d} s \leq 0 .
$$

Moreover, by $L^{2}$-weak convergence of $\dot{x}_{n}$ to $\dot{x}$, we have

$$
\lim _{n \rightarrow \infty} \int_{\widehat{a}}^{\widehat{b}} g\left(\dot{x}, \dot{x}_{n}-\dot{x}\right) \mathrm{d} s=0
$$

and from the last two relations above it follows that

$$
\limsup _{n \rightarrow \infty} \int_{\widehat{a}}^{\widehat{b}} g\left(\dot{x}_{n}-\dot{x}, \dot{x}_{n}-\dot{x}\right) \mathrm{d} s \leq 0,
$$

which proves (6.9), and the proof is complete.

Now we need to extend the above descent direction, obtained on a portion of the curve $x_{\mid[a, b]}$, to a neighborhood of it. Towards this goal some preliminary notations and results are needed. 
First observe that, due to the compactness of $\left.\left.\phi^{-1}(]-\infty, \delta_{0}\right]\right)$ and the form of Christoffel symbols, there exists two positive constants $\ell_{0}, L_{0}$ such that, denoting by $\|\cdot\|_{E}$ the Euclidean norm and by $\|\cdot\|$ the $g$-norm,

$$
\ell_{0}\|v\|_{E}^{2} \leq\|v\|^{2} \leq L_{0}\|v\|_{E}^{2}
$$

for any $v \in \mathbb{R}^{N}$, for any $\left.\left.x \in \phi^{-1}(]-\infty, \delta_{0}\right]\right)$. Moreover denoting by $g_{x}$ the metric tensor $g$ evaluated on $T_{x} M$, there exists a constant $G_{0}>0$ such that

$$
\left|g_{x}\left(v_{1}, v\right)-g_{z}\left(v_{2}, v\right)\right| \leq G_{0}\left(\left\|v_{1}-v_{2}\right\|_{E}\|v\|_{E}+\|x-z\|_{E}\left\|v_{1}\right\|_{E}\|v\|_{E}\right),
$$

for any $\left.\left.x, z \in \phi^{-1}(]-\infty, \delta_{0}\right]\right)$ and for any $v_{1}, v_{2}, v \in \mathbb{R}^{N}$. Finally we see also that there exists $L_{1}=L_{1}\left(M_{0}\right)$ such that

$$
\begin{aligned}
& \left(\int_{a}^{b}\left\|\frac{\mathrm{D}}{\mathrm{d} s} V\right\|_{E}^{2}, \mathrm{~d} s\right)^{1 / 2} \leq L_{1}\|V\|_{a, b}, \\
& \text { for any } x \in \mathfrak{M}_{0} \text { and }[a, b] \subset[0,1] \text { such that } \frac{1}{2} \int_{a}^{b} g(\dot{x}, \dot{x}) \mathrm{d} s \leq M_{0}, \\
& \qquad \text { and for any vector field } V \in H^{1}\left([a, b], \mathbb{R}^{N}\right) \text { along } x .
\end{aligned}
$$

Now, for any $a, b \in[0,1]$, denote by $I_{a, b}$ the interval $[a, b]$ (possibly reduced to a single point) if $b \geq a$ and the interval $[b, a]$ if $b<a$. For any intervals $[a, b]$ and $[\alpha, \beta]$ we set:

$$
\mathcal{D}(x, \alpha, \beta, a, b)=\frac{1}{2} \int_{I_{a, \alpha} \cup I_{b, \beta}} g(\dot{x}, \dot{x}) \mathrm{d} t .
$$

The following preparatory lemma holds.

Lemma 6.3. Fix $K>0$. For any $x, z \in \mathfrak{M}_{0}, \quad \forall[a, b]$ and $\left[a_{z}, b_{z}\right]$ in $[0,1], \quad \forall V \in$ $H^{1}\left([0,1], \mathbb{R}^{N}\right)$, if $\frac{1}{2} \int_{a}^{b} g(\dot{x}, \dot{x}) \mathrm{d} s \leq M_{0}, \mathcal{D}\left(x, a_{z}, b_{z}, a, b\right) \leq K$ and $\left[a_{z}, b_{z}\right] \cap[a, b] \neq \emptyset$, it is

$$
\begin{aligned}
\mid \int_{a}^{b} g_{x}\left(\dot{x}, \frac{\mathrm{D}}{\mathrm{d} s} V\right) \mathrm{d} s & -\int_{a_{z}}^{b_{z}} g_{z}\left(\dot{z}, \frac{\mathrm{D}}{\mathrm{d} s} V\right) \mathrm{d} s \mid \leq \\
& \sqrt{2}\left(\sqrt{L_{0} K}+G_{0}\|x-z\|_{a_{z}, b_{z}}\left(1+\sqrt{\frac{M_{0}+K}{\ell_{0}}}\right)\right) L_{1}\|V\|_{0,1},
\end{aligned}
$$


Proof. We have:

$$
\begin{gathered}
\left|\int_{a}^{b} g_{x}\left(\dot{x}, \frac{\mathrm{D}}{\mathrm{d} s} V\right) \mathrm{d} s-\int_{a_{z}}^{b_{z}} g_{z}\left(\dot{z}, \frac{\mathrm{D}}{\mathrm{d} s} V\right) \mathrm{d} s\right| \leq \\
\left|\int_{a}^{b} g_{x}\left(\dot{x}, \frac{\mathrm{D}}{\mathrm{d} s} V\right) \mathrm{d} s-\int_{a_{z}}^{b_{z}} g_{x}\left(\dot{x}, \frac{\mathrm{D}}{\mathrm{d} s} V\right) \mathrm{d} s\right|+\left|\int_{a_{z}}^{b_{z}} g_{x}\left(\dot{x}, \frac{\mathrm{D}}{\mathrm{d} s} V\right) \mathrm{d} s-\int_{a_{z}}^{b_{z}} g_{z}\left(\dot{z}, \frac{\mathrm{D}}{\mathrm{d} s} V\right) \mathrm{d} s\right| \\
\leq \int_{I_{a, a_{z}} \cup I_{b, b_{z}}}\|\dot{x}\|\left\|\frac{\mathrm{D}}{\mathrm{d} s} V\right\| \mathrm{d} s+ \\
G_{0}\left[\int_{a_{z}}^{b_{z}}\|\dot{x}-\dot{z}\|_{E}\left\|\frac{\mathrm{D}}{\mathrm{d} s} V\right\|_{E} \mathrm{~d} s+\int_{a_{z}}^{b_{z}}\|x-z\|_{E}\|\dot{x}\|_{E}\left\|\frac{\mathrm{D}}{\mathrm{d} s} V\right\|_{E} \mathrm{~d} s\right] \leq \\
\left(\int_{I_{a, a_{z}} \cup I_{b, b_{z}}} g(\dot{x}, \dot{x}) \mathrm{d} s\right)^{1 / 2}\left(\int_{0}^{1}\left\|\frac{\mathrm{D}}{\mathrm{d} s} V\right\|_{E}^{2} \mathrm{~d} s\right)^{1 / 2} \sqrt{L_{0}}+ \\
G_{0}\|\dot{x}-\dot{z}\|_{L^{2}\left(\left[a_{z}, b_{z}\right], \mathbb{R}^{N}\right)}\left(\int_{0}^{1}\left\|\frac{\mathrm{D}}{\mathrm{d} s} V\right\|_{E}^{2}\right)^{1 / 2}+ \\
G_{0}\|x-z\|_{L^{\infty}\left(\left[a_{z}, b_{z}\right], \mathbb{R}^{N}\right)}\left(\int_{a_{z}}^{b_{z}}\|\dot{x}\|_{E}^{2} \mathrm{~d} s\right)^{1 / 2}\left(\int_{0}^{1}\left\|\frac{\mathrm{D}}{\mathrm{d} s} V\right\|_{E}^{2}\right)^{1 / 2} .
\end{gathered}
$$

Second inequality above is due to Schwarz inequality, the definition of $I_{a, \alpha}$, and (6.14), while the third is given by Hölder inequality and (6.13). Now by 1.8 we have

$$
\|\dot{x}-\dot{z}\|_{L^{2}\left(\left[a_{z}, b_{z}\right], \mathbb{R}^{N}\right)} \leq \sqrt{2}\|x-z\|_{a_{z}, b_{z}}, \quad\|x-z\|_{L^{\infty}\left(\left[a_{z}, b_{z}\right], \mathbb{R}^{N}\right)} \leq\|x-z\|_{a_{z}, b_{z}} .
$$

Therefore by (6.16), assumption $\mathcal{D}\left(x, a_{z}, b_{z}, a, b\right) \leq K$ and 6.15) it is :

$$
\begin{aligned}
\left|\int_{a}^{b} g_{x}\left(\dot{x}, \frac{\mathrm{D}}{\mathrm{d} s} V\right) \mathrm{d} s-\int_{a_{z}}^{b_{z}} g_{z}\left(\dot{z}, \frac{\mathrm{D}}{\mathrm{d} s} V\right) \mathrm{d} s\right| \leq \\
\quad\left(\sqrt{2 L_{0} K}+G_{0}\|x-z\|_{a_{z}, b_{z}}\left(\sqrt{2}+\left(\int_{a_{z}}^{b_{z}}\|\dot{x}\|_{E} \mathrm{~d} s\right)^{1 / 2}\right)\right) L_{1}\|V\|_{0,1},
\end{aligned}
$$

and finally, since

$$
\begin{aligned}
& \left(\int_{a_{z}}^{b_{z}}\|\dot{x}\|_{E}^{2} \mathrm{~d} s\right)^{1 / 2} \leq \frac{1}{\sqrt{\ell_{0}}}\left(\int_{a_{z}}^{b_{z}} g(\dot{x}, \dot{x}) \mathrm{d} s\right)^{1 / 2}, \\
& \left|\frac{1}{2} \int_{a_{z}}^{b_{z}} g(\dot{x}, \dot{x}) \mathrm{d} s-\frac{1}{2} \int_{a}^{b} g(\dot{x}, \dot{x}) \mathrm{d} s\right| \leq \frac{1}{2} \int_{I_{a, a_{z}} \cup I_{b, b z}} g(\dot{x}, \dot{x}) \mathrm{d} s \leq K,
\end{aligned}
$$

and

$$
\frac{1}{2} \int_{a}^{b} g(\dot{x}, \dot{x}) \mathrm{d} s \leq M_{0},
$$

we obtain the proof.

Let now $\mu(r)$ be as in Proposition 6.1, and $L_{0}$ and $L_{1}$ be the constants given by 6.13 and (6.15) respectively. Set

$$
E(r)=\frac{\mu^{2}(r)}{32 L_{1}^{2} L_{0}}
$$


Proposition 6.4. Fix $r>0$. Let $\theta(r)$ and $\mu(r)$ be as in Proposition 6.1. Then there exist $\rho(r)>0$ with the following property.

For all $x \in \mathfrak{M}_{0}$ and for all $[a, b] \subset[0,1]$ satisfying (6.2) (6.3) and (6.4), let $V_{x}$ be as in Proposition 6.1. Extend it to $[0,1]$ so that $V_{x}(s)=V(x(a))$ on $[0, a]$ and $V_{x}(s)=V(x(b))$ on $[b, 1]$. Then there exists $\Delta(x)=\Delta(x,[a, b])>0$ such that:

(1) for any $z \in \mathfrak{M}_{0}$ satisfying $\|x-z\|_{L^{\infty}\left([0,1], \mathbb{R}^{N}\right)}<\rho(r)$ the following conditions hold:

(a) for any $\left.\delta \in] 0, \delta_{0}\right]$, for any $\left[c_{z}, d_{z}\right] \subset[a-\Delta(x), b+\Delta(x)] \cap[0,1], \delta$-interval for $z$, and for all $s \in\left[c_{z}, d_{z}\right]$ such that $\phi(z(s))=-\delta$, it is

$$
g\left(\nabla \phi(z(s)), V_{x}(s)\right) \geq \frac{\theta(r)}{2}\left\|V_{x}\right\|_{a, b} ;
$$

(b) $g\left(\nabla \phi(z(s)), V_{x}(s)\right) \geq \frac{\theta(r)}{2}\left\|V_{x}\right\|_{a, b}$ for all $s \in[a-\Delta(x), b+\Delta(x)] \cap[0,1]$ with $\phi(z(s))=0$;

(2) for all $z \in \mathfrak{M}_{0}$ and for all $\left[a_{z}, b_{z}\right] \in \mathcal{I}_{z}$, such that $\|x-z\|_{a_{z}, b_{z}}<\rho(r),\left[a_{z}, b_{z}\right] \cap$ $[a, b] \neq \emptyset$, and $\mathcal{D}\left(x, a_{z}, b_{z}, a, b\right) \leq E(r)$, it is

$$
\int_{a_{z}}^{b_{z}} g\left(\dot{z}, \frac{\mathrm{D}}{\mathrm{d} s} V_{x}\right) \mathrm{d} s \leq-\frac{\mu(r)}{2}\left\|V_{x}\right\|_{a, b} .
$$

Remark 6.5. By property (2) of Proposition 6.4 and the definition of $D\left(x, a_{z}, b_{z}, a, b\right)$ (cf. (6.16)) we see that the number $E(r)$ gives a bound on the admissible difference between the energy of $x_{\mid[a, b]}$ and $x_{\mid\left[a_{z}, b_{z}\right]}$, to obtain a rate of decrease $\mu(r) / 2$ for the quantity $\frac{1}{2} \int_{a_{z}}^{b_{z}} g(\dot{z}, \dot{z}) \mathrm{d} s$, when $\|x-z\|_{a_{z}, b_{z}}<\rho(r)$.

Remark 6.6. By Proposition 6.4 we see that we can choose $V_{\mathcal{R} x}$ so that $V_{\mathcal{R} x}=\mathcal{R} V_{x}$.

Proof of Proposition 6.4. Let $\kappa(r)$ given by Proposition 6.1. In order to prove (1a), let us consider $\left[c_{z}, d_{z}\right], \delta$-interval for $z$.

Suppose $\delta \leq \frac{\kappa(r)}{2}$. Since $\phi(x(a)=\phi(x(b))=0$ it is immediately seen that we can choose $\Delta_{1}(x)>0$ and $\rho_{1}(r)>0$ such that

$$
\begin{array}{r}
{\left[c_{z}, d_{z}\right] \subset\left[a-\Delta_{1}(x), b+\Delta_{1}(x)\right] \cap[0,1] \text { and }\|x-z\|_{L^{\infty}\left([0,1], \mathbb{R}^{N}\right)}<\rho_{1}(r) \Longrightarrow} \\
\phi\left(x\left(\left[c_{z}, d_{z}\right]\right) \subset[-\kappa(r), \kappa(r)] .\right.
\end{array}
$$

Therefore by (2) of Proposition 6.1 we have

$$
g\left(\nabla \phi(x(s)), V_{x}(s)\right) \geq \theta(r)\left\|V_{x}\right\|_{a, b} \text { for all } s \in\left[c_{z}, d_{z}\right],
$$

so there exists $\left.\left.\rho_{2}(r) \in\right] 0, \rho_{1}(r)\right]$ such that if $\|x-z\|_{L^{\infty}\left([0,1], \mathbb{R}^{N}\right)}<\rho_{2}(r)$ then

$$
\left.\left.g\left(\nabla \phi(z(s)), V_{x}\right) s\right)\right) \geq \frac{\theta(r)}{2}\left\|V_{x}\right\|_{a, b} \text { for all } s \in\left[c_{z}, d_{z}\right] .
$$

Now assume $\left.\delta \in] \frac{\kappa(r)}{2}, \delta_{0}\right]$. Then there exists $\left.\left.\rho_{3}(r) \in\right] 0, \rho_{2}(r)\right]$ and $\left.\left.\Delta_{2}(x) \in\right] 0, \Delta_{1}(x)\right]$ such that

$$
\begin{gathered}
{\left[c_{z}, d_{z}\right] \subset\left[a-\Delta_{2}(x), b+\Delta_{2}(x)\right] \cap[0,1],\|x-z\|_{L^{\infty}\left([0,1], \mathbb{R}^{N}\right)}<\rho_{3}(r) \text { and } s \in\left[c_{z}, d_{z}\right]} \\
\quad \operatorname{imply} \phi(x(s)) \in[-\kappa(r), \kappa(r)] \text { or } \\
s \in[\alpha, \beta] \subset[a, b], \text { where }[\alpha, \beta] \text { is a }\left(\delta_{1}, \frac{\kappa(r)}{8}\right) \text {-interval for } x \text { with } \delta_{1} \geq \frac{\kappa(r)}{4} .
\end{gathered}
$$

Then by (1) and (2) of Proposition 6.1, (6.18) is satisfied for any $\left[c_{z}, d_{z}\right] \subset[a-$ $\left.\Delta_{2}(x), b+\Delta_{2}(x)\right] \cap[0,1], \delta$-interval for $z$ and for any $\left.\left.\delta \in\right] 0, \delta_{0}\right]$. Hence there exists $\left.\left.\rho_{4}(r) \in\right] 0, \rho_{3}(r)\right]$ such that also 6.19$)$ is satisfied for any $\left[c_{z}, d_{z}\right] \subset\left[a-\Delta_{2}(x), b+\right.$ $\left.\Delta_{2}(x)\right] \cap[0,1], \delta$-interval for $z$ and $\left.\left.\delta \in\right] 0, \delta_{0}\right]$. This proves (1a) with $\rho(r)=\rho_{4}(r)$ and $\Delta(x)=\Delta_{2}(x)$. Clearly we can also choose $\rho(r)$ and $\Delta(x)$ so that $\|x-z\|_{L^{\infty}\left([0,1], \mathbb{R}^{N}\right)}<$ 
$\rho(r), s \in[a-\Delta(x), b+\Delta(x)]$, and $\phi(z(s))=0$ imply $\phi(x(s) \in[-\kappa(r), \kappa(r)]$, from which we immediately deduce that we can choose $\rho(r)$ and $\Delta(x)$ so that also $(1 \mathrm{~b})$ holds.

Finally, to show (2) observe that, if $z \in \mathfrak{M}_{0},\|x-z\|_{a_{z}, b_{z}}<\rho(r),\left[a_{z}, b_{z}\right] \cap[a, b] \neq \emptyset$ and $\mathcal{D}\left(x, a_{z}, b_{z}, a, b\right) \leq E(r),(E(r)$ given by (6.17) $)$, by Lemma 6.3, we have

$$
\begin{aligned}
\int_{a_{z}}^{b_{z}} g\left(\dot{z}, \frac{\mathrm{D}}{\mathrm{d} s} V_{x}\right) \mathrm{d} s \leq \int_{a}^{b} g\left(\dot{x}, \frac{\mathrm{D}}{\mathrm{d} s} V_{x}\right) \mathrm{d} s+ \\
\quad \sqrt{2}\left(\sqrt{L_{0} E(r)}+G_{0}\|x-z\|_{a_{z}, b_{z}}\left(1+\sqrt{\frac{M_{0}+E(r)}{\ell_{0}}}\right)\right) L_{1}\left\|V_{x}\right\|_{0,1} .
\end{aligned}
$$

Since $\left\|V_{x}\right\|_{0,1}=\left\|V_{x}\right\|_{a, b}$, applying (3) of Proposition 6.1 we have

$$
\begin{aligned}
& \int_{a_{z}}^{b_{z}} g\left(\dot{z}, \frac{\mathrm{D}}{\mathrm{d} s} V_{x}\right) \mathrm{d} s \leq \\
& \quad\left[-\mu(r)+\sqrt{2}\left(\sqrt{L_{0} E(r)}+G_{0}\|x-z\|_{a_{z}, b_{z}}\left(1+\sqrt{\frac{M_{0}+E(r)}{\ell_{0}}}\right)\right)\right] L_{1}\left\|V_{x}\right\|_{a, b},
\end{aligned}
$$

therefore we can choose $\rho(r)$ such that property (2) is satisfied, because $L_{1} \sqrt{2 L_{0} E(r)}=$ $\frac{\mu(r)}{4}$ (see 6.17$)$.

\section{The PALAis-SMALE FOR $\mathcal{V}^{-}$-CRITICAL PORTIONS AND THE NOTION OF TOPOLOGICAL NON ESSENTIAL INTERVAL}

Before to describe the class of admissible homotopies we need to give the Palais-Smale version for " $\mathcal{V}^{-}-$critical portions". Towards this goal we need some preliminary notations and results.

Throughout this section we will denote by

$$
\pi: \phi^{-1}\left(\left[-\delta_{0}, 0\right]\right) \longrightarrow \phi^{-1}(0)
$$

the "projection" onto $\partial \Omega$ along orthogonal geodesics (cf. (1.10)). Thanks to Remark 4.5 a simple contradiction argument shows that the following properties are satisfied by irregular variationally critical portions of first type (see also Corollary 4.8):

Lemma 7.1. There exists $\bar{\gamma}>0$ and $\left.\delta_{1} \in\right] 0, \delta_{0}[$ such that, for all $\left.\delta \in] 0, \delta_{1}\right]$, for any $x \in \mathfrak{M}_{0}$ such that $x_{[[a, b]}$ is an irregular variationally critical portion of first type with $\frac{1}{2} \int_{a}^{b} g(\dot{x}, \dot{x}) \mathrm{d} s<M_{0}$ and for any interval $[\alpha, \beta] \subset[a, b]$ including a cusp interval $\left[t_{1}, t_{2}\right]$ satisfying (4.4) the following inequality holds:

$$
\max \left\{\|x(\beta)-\pi(x(\alpha))\|_{E},\|x(\alpha)-\pi(x(\beta))\|_{E}\right\} \geq(1+2 \bar{\gamma})\|\pi(x(\beta))-\pi(x(\alpha))\|_{E},
$$

(recall that $\|\cdot\|_{E}$ denotes the Euclidean norm).

The following Lemma says that curves satisfying (7.1) are far away from those that satisfy (5.2): in other words, they are far to be critical with respect to $\mathcal{V}^{-}$. In particular, the set of irregular variationally critical portions of first type consists of curves at which the value of the functional can be decreased by deforming in the directions of $\mathcal{V}^{-}$, as we shall see later.

Let $\bar{\gamma}$ be as in Lemma 7.1 .

Lemma 7.2. There exists $\left.\delta_{2} \in\right] 0, \delta_{0}[$ with the following property: for any $\left.\delta \in] 0, \delta_{2}\right]$, for any $[a, b] \subset \mathbb{R}$ and for any $y \in H^{1}([a, b], \bar{\Omega})$ satisfying (5.2) and such that

$$
\phi(y(a))=\phi(y(b))=-\delta, \phi(y(\bar{t}))=0 \text { for some } \bar{t} \in] a, b[,
$$


the following inequality holds:

(7.2)

$$
\max \left\{\|y(b)-\pi(y(a))\|_{E},\|y(a)-\pi(y(b))\|_{E}\right\} \leq\left(1+\frac{\bar{\gamma}}{2}\right)\|\pi(y(b))-\pi(y(a))\|_{E} .
$$

Proof. Let $y \in H^{1}([0,1], \bar{\Omega})$ be fixed. If $y$ satisfies (5.2) then it is of class $C^{1}$. Moreover $\phi(y(s)) \leq 0$ on $[a, b]$ so that $g(\nabla \phi(y(s)), \dot{y}(s))=0$ for all $s$ with $\phi(y(s))=0$. Thanks to the conservation law $g(\dot{y}, \dot{y})=$ constant (see Lemma 5.4), we have that $\dot{y}(s) \neq 0$ for all $s \in$ $[a, b]$, since $\phi(x(a))=-\delta$ and $\phi(x(\bar{t}))=0$. Denote by $\tilde{y}$ the arc-length reparameterization of $y$ and let $[\tilde{a}, \tilde{b}]$ be the interval such that $\phi(\tilde{y}(\tilde{a}))=\phi(\tilde{y}(\tilde{b}))=-\delta$. Note that $[\tilde{a}, \tilde{b}]$ is uniquely determined, (up to translations of the affine parameter), since the strong concavity condition implies $g(\nabla \phi(y(a)), \dot{y}(a))>0$ and $g(\nabla \phi(y(b)), \dot{y}(b))<0$. In addition, the strong concavity condition also ensures the existence of $\tilde{c}>0$ such that $|\tilde{b}-\tilde{a}| \leq \tilde{c}$ for any $\tilde{y}$ as above. Then, for any $y$, we can assume $g(\dot{y}, \dot{y})=1 \forall s \in[a, b]$ without loss of generality (observe that $(7.2)$ is a condition independent on the parameterization of $y$ ).

By contradiction, assume now that the claim does not hold. Then, there exists a sequence of intervals $\left[a_{n}, b_{n}\right]$ of bounded length and a sequence of functions $y_{n}$ such that $y_{n \mid\left[a_{n}, b_{n}\right]}$ satisfies (5.2), $g\left(\dot{y}_{n}, \dot{y}_{n}\right)=1$ on $\left[a_{n}, b_{n}\right],-\delta_{n}=\phi\left(y_{n}\left(a_{n}\right)\right)=\phi\left(y_{n}\left(b_{n}\right)\right)$, $\left(\delta_{n} \rightarrow 0^{+}\right), \phi\left(y_{n}\left(t_{n}\right)\right)=0$ for some $\left.t_{n} \in\right] a_{n}, b_{n}[$ and (possibly reversing the orientation of $\left.y_{n}\right)$ :

$$
\left\|y_{n}\left(a_{n}\right)-\pi\left(y_{n}\left(b_{n}\right)\right)\right\|_{E}>\left(1+\frac{\bar{\gamma}}{2}\right)\left\|\pi\left(y_{n}\left(a_{n}\right)\right)-\pi\left(y_{n}\left(b_{n}\right)\right)\right\|_{E} .
$$

Since $\left|b_{n}-a_{n}\right|$ is bounded, up to a translation of the parameter, we can assume $a_{n}$ and $b_{n}$ bounded. Moreover $\bar{\Omega}$ is compact, so by Lemma 5.4, using local coordinates expression of the covariant derivative (involving Christoffel symbols, cf. [卂]) we obtain the existence of a constant $C>0$ independent of $n$ such that

$$
\left\|\ddot{y}_{n}(s)\right\|_{E} \leq C g\left(\dot{y}_{n}(s), \dot{y}_{n}(s)\right) \text { for a.e. } s \in\left[a_{n}, b_{n}\right] .
$$

But $g\left(\dot{y}_{n}(s), \dot{y}_{n}(s)=1\right.$ for any $s \in\left[a_{n}, b_{n}\right]$, then

$$
\left\|\ddot{y}_{n}(s)\right\|_{E} \leq C \text { for a.e. } s \in\left[a_{n}, b_{n}\right] .
$$

Now, let $\left.t_{n}^{-} \in\right] a_{n}, b_{n}$ [ be the first instant at which the function $t \mapsto \phi\left(y_{n}(t)\right)$ vanishes. By Taylor expansion we have

$$
\left\|y_{n}(s)-y_{n}\left(t_{n}^{-}\right)-\dot{y}_{n}\left(t_{n}^{-}\right)\left(s-t_{n}^{-}\right)\right\|_{E} \leq \frac{C}{2}\left(s-t_{n}^{-}\right)^{2}
$$

for any $s \in\left[a_{n}, t_{n}^{-}\right]$. The argument is analogous in the interval $\left[t_{n}^{+}, b_{n}\right]$, where $t_{n}^{+}$denotes the last instant when $\phi\left(y_{n}(\cdot)\right)$ vanishes. Now, the same estimates, applied to $\pi\left(y_{n}\right)$, give the existence of a constant $C_{1}>0$ such that:

$$
\left\|\pi\left(y_{n}(s)\right)-\pi\left(y_{n}\left(t_{n}^{-}\right)\right)-\mathrm{d} \pi\left(y_{n}\left(t_{n}^{-}\right)\right)\left[\dot{y}_{n}\left(t_{n}^{-}\right)\right]\left(s-t_{n}^{-}\right)\right\|_{E} \leq C_{1}\left(s-t_{n}^{-}\right)^{2} .
$$

But for all $z \in \partial \Omega, \pi(z)=z$ and $\mathrm{d} \pi(z)$ is the identity map, and therefore

$$
\left\|\pi\left(y_{n}(s)\right)-y_{n}\left(t_{n}^{-}\right)-\dot{y}_{n}\left(t_{n}^{-}\right)\left(s-t_{n}^{-}\right)\right\|_{E} \leq C_{1}\left(s-t_{n}^{-}\right)^{2}
$$

for all $s \in\left[a_{n}, t_{n}^{-}\right]$, and, analogously, for all $s$ in $\left[t_{n}^{+}, b_{n}\right]$. Now

$$
\begin{aligned}
& \left\|\pi\left(y_{n}\left(a_{n}\right)\right)-\pi\left(y_{n}\left(b_{n}\right)\right)\right\|_{E}= \\
& \quad \|\left[\pi\left(y_{n}\left(a_{n}\right)\right)-y_{n}\left(t_{n}^{-}\right)-\dot{y}_{n}\left(t_{n}^{-}\right)\left(a_{n}-t_{n}^{-}\right)\right]+\left[y_{n}\left(t_{n}^{-}\right)+\dot{y}_{n}\left(t_{n}^{-}\right)\left(a_{n}-t_{n}^{-}\right)\right] \\
& \quad-\left[y_{n}\left(t_{n}^{+}\right)+\dot{y}_{n}\left(t_{n}^{+}\right)\left(b_{n}-t_{n}^{+}\right)\right]+\left[y_{n}\left(t_{n}^{+}\right)+\dot{y}_{n}\left(t_{n}^{+}\right)\left(b_{n}-t_{n}^{+}\right)-\pi\left(y_{n}\left(b_{n}\right)\right)\right] \|_{E},
\end{aligned}
$$


and, similarly,

$$
\begin{aligned}
& \left\|y_{n}\left(a_{n}\right)-\pi\left(y_{n}\left(b_{n}\right)\right)\right\|_{E}= \\
& \quad \|\left[y_{n}\left(a_{n}\right)-y_{n}\left(t_{n}^{-}\right)-\dot{y}_{n}\left(t_{n}^{-}\right)\left(a_{n}-t_{n}^{-}\right)\right]+\left[y_{n}\left(t_{n}^{-}\right)+\dot{y}_{n}\left(t_{n}^{-}\right)\left(a_{n}-t_{n}^{-}\right)\right] \\
& \quad-\left[y_{n}\left(t_{n}^{+}\right)+\dot{y}_{n}\left(t_{n}^{+}\right)\left(b_{n}-t_{n}^{+}\right)\right]+\left[y_{n}\left(t_{n}^{+}\right)+\dot{y}_{n}\left(t_{n}^{+}\right)\left(b_{n}-t_{n}^{+}\right)-\pi\left(y_{n}\left(b_{n}\right)\right)\right] \|_{E} .
\end{aligned}
$$

The strong concavity assumption implies that $\left(t_{n}^{-}-a_{n}\right) \rightarrow 0$ and $\left(b_{n}-t_{n}^{+}\right) \rightarrow 0$ as $n \rightarrow+\infty$ (since $\delta_{n} \rightarrow 0$ ). Then, by (7.4) and (7.5) (and their analogues in $\left[t_{n}^{+}, b_{n}\right]$ ) we have

$$
\lim _{n \rightarrow+\infty} \frac{\left\|y_{n}\left(a_{n}\right)-\pi\left(y_{n}\left(b_{n}\right)\right)\right\|_{E}}{\left\|\pi\left(y_{n}\left(a_{n}\right)\right)-\pi\left(y_{n}\left(b_{n}\right)\right)\right\|_{E}}=1
$$

unless (up to subsequences)

$$
\lim _{n \rightarrow+\infty}\left(y_{n}\left(t_{n}^{-}\right)-y_{n}\left(t_{n}^{+}\right)\right)=0, \quad \lim _{n \rightarrow+\infty}\left(\dot{y}_{n}\left(t_{n}^{-}\right)+\dot{y}_{n}\left(t_{n}^{+}\right)\right)=0,
$$

(recall that $a_{n}-t_{n}^{-}<0$ and $b_{n}-t_{n}^{+}>0$ ) that is the only case when (7.6) above cannot be immediately deduced. Therefore, to conclude the proof — recalling (7.3) — it suffices to prove that (7.7) cannot hold, using the fact that $g\left(\dot{y}_{n}, \dot{y}_{n}\right) \equiv 1$ on $\left[a_{n}, b_{n}\right]$. Suppose by contradiction that (7.7) holds. First, observe that

$$
\left\|\dot{y}_{n}\left(t_{n}^{+}\right)-\dot{y}_{n}\left(t_{n}^{-}\right)\right\|_{E} \leq \int_{t_{n}^{-}}^{t_{n}^{+}}\left\|\ddot{y}_{n}(s)\right\|_{E} \mathrm{~d} s \leq C\left(t_{n}^{+}-t_{n}^{-}\right)
$$

and therefore, by the second property in (7.7) and the fact that $g\left(\dot{y}_{n}, \dot{y}_{n}\right) \equiv 1$ on $\left[a_{n}, b_{n}\right]$, we deduce that

$$
\inf _{n \in \mathbb{N}}\left(t_{n}^{+}-t_{n}^{-}\right)>0 .
$$

Since the sequence $y_{n}$ is bounded in $H^{2, \infty}\left(\left[a_{n}, b_{n}\right], \bar{\Omega}\right),\left[t_{n}^{-}, t_{n}^{+}\right] \subset\left[a_{n}, b_{n}\right]$ and $b_{n}, a_{n}$ are bounded, up to subsequence we deduce the existence of an interval $\left[t^{-}, t^{+}\right]\left(t^{-}<t^{+}\right)$and $y \in H^{2, \infty}\left(\left[t^{-}, t^{+}\right], \bar{\Omega}\right)$ such that (recall we are assuming that $(7.7)$ holds)

$$
\begin{aligned}
& y_{\left[t^{-}, t^{+}\right]} \text {satisfies }[5.2), \\
& g(\dot{y}, \dot{y}) \equiv 1 \quad \text { on }\left[t^{-}, t^{+}\right], \\
& y\left(t^{-}\right)=y\left(t^{+}\right), \quad \text { (by first property in }([7.7) \text { ), } \\
& \dot{y}\left(t^{+}\right)=-\dot{y}\left(t^{-}\right), \quad \text { (by second property in }([7.7) \text { ). }
\end{aligned}
$$

Then by the uniqueness of the solution of the Cauchy problem for the "free" geodesic equation $\left(\frac{\mathrm{D}}{\mathrm{d} t} \dot{y}=0\right)$ and for the "constrained" geodesic equation (5.8), we have:

$$
y\left(\left(t^{+}+t^{-}\right)-s\right)=y(s) \quad \forall s \in\left[t^{-}, t^{+}\right]
$$

since the functions on the two side of the equality are solutions of the same differential equation with the same initial data. But $y$ is of class $C^{1}$ while

$$
\dot{y}\left(\frac{t^{+}+t^{-}}{2}\right)=-\dot{y}\left(\frac{t^{+}+t^{-}}{2}\right),
$$

from which we deduce $\dot{y}\left(\frac{t^{+}+t^{-}}{2}\right)=0$, in contradiction with $g(\dot{y}, \dot{y}) \equiv 1$ on $\left[t^{-}, t^{+}\right]$.

Using vector fields in $\mathcal{V}^{-}(x), x \in \mathfrak{M}$, we shall build a flow $H_{0}$ moving away from the set of irregular variationally critical portions of first type, without increasing the energy functional. To this aim let $\pi, \bar{\gamma}, \delta_{1}, \delta_{2}$ be chosen as in Lemma 7.1 and 7.2, and set

$$
\bar{\delta}=\min \left\{\delta_{1}, \delta_{2}\right\} \text {. }
$$

Let us give the following:

Definition 7.3. Let $x \in \mathfrak{M}_{0},[a, b] \in \mathcal{J}_{x}^{0}$ and $[\alpha, \beta] \subset[a, b]$. We say that $x$ is $\bar{\delta}$-close to $\partial \Omega$ on $[\alpha, \beta]$ if the following situation occurs: 
(1) $\phi(x(\alpha))=\phi(x(\beta))=-\bar{\delta}$;

(2) $\phi(x(s)) \geq-\bar{\delta}$ for all $s \in[\alpha, \beta]$;

(3) there exists $\left.s_{0} \in\right] \alpha, \beta\left[\right.$ such that $\phi\left(x\left(s_{0}\right)\right)>-\bar{\delta}$;

(4) $[\alpha, \beta]$ is minimal with respect to properties (11), (2) and (3).

If $x$ is $\bar{\delta}$-close to $\partial \Omega$ on $[\alpha, \beta]$, the maximal proximity of $x$ to $\partial \Omega$ on $[\alpha, \beta]$ is defined to be the quantity

$$
\mathfrak{p}_{\alpha, \beta}^{x}=\max _{s \in[\alpha, \beta]} \phi(x(s)) .
$$

Given an interval $[\alpha, \beta]$ where $x$ is $\bar{\delta}$-close to $\partial \Omega$, we define the following constant, which is a sort of measure of how much the curve $x_{\mid[\alpha, \beta]}$ fails to flatten along $\partial \Omega$ :

Definition 7.4. The bending constant of $x$ on $[\alpha, \beta]$ is defined by:

$$
\mathfrak{b}_{\alpha, \beta}^{x}=\frac{\max \left\{\|x(\beta)-\pi(x(\alpha))\|_{E},\|x(\alpha)-\pi(x(\beta))\|_{E}\right\}}{\|\pi(x(\alpha))-\pi(x(\beta))\|_{E}} \in \mathbb{R}^{+} \cup\{+\infty\},
$$

where $\pi$ denotes the projection onto $\partial \Omega$ along orthogonal geodesics.

We observe that $\mathfrak{b}_{\alpha, \beta}^{x}=+\infty$ if and only if $x(\alpha)=x(\beta)$.

Let $\bar{\gamma}$ be as in Lemma 7.1. Our next result is the counterpart of the result in Propositions 6.1 and 6.4, and it will be used to define a flow that averts from paths in the set of irregular variationally critical portions of first type: if the bending constant of a path $y_{\mid[\alpha, \beta]}$ is greater than or equal to $1+\bar{\gamma}$, then the energy functional $f_{\alpha, \beta}$ can be decreased in a neighborhood of $y_{\mid[\alpha, \beta]}$ keeping the endpoints $y(\alpha)$ and $y(\beta)$ fixed and moving far away from $\partial \Omega$.

First we need the following

Definition 7.5. An interval $[\tilde{\alpha}, \tilde{\beta}]$ is called summary interval for $x \in \mathfrak{M}_{0}$ if it is the smallest interval included in $[a, b] \in \mathcal{I}_{x}^{0}$ and including all the intervals $[\alpha, \beta]$ such that

- $x$ is $\bar{\delta}$-close to $\partial \Omega$ on $[\alpha, \beta]$,

- $b_{\alpha, \beta}^{x} \geq 1+\bar{\gamma}$.

Proposition 7.6. There exist positive constants $\left.\sigma_{0} \in\right] 0, \bar{\delta} / 2\left[, \varepsilon_{0} \in\right] 0, \bar{\delta}-2 \sigma_{0}\left[, \rho_{0}, \theta_{0}\right.$ and $\mu_{0}$ such that for all $y \in \mathfrak{M}_{0}$, for all $[a, b] \in \mathcal{I}_{y}^{0}$ satisfying $\frac{1}{2} \int_{a}^{b} g(\dot{y}, \dot{y}) d s \leq M_{0}$ and for all $[\tilde{\alpha}, \tilde{\beta}]$ summary interval for $y$ including an interval $[\alpha, \beta]$ such that :

$$
y \text { is } \bar{\delta} \text {-close to } \partial \Omega \text { on }[\alpha, \beta], \mathfrak{b}_{\alpha, \beta}^{y} \geq 1+\bar{\gamma}, \mathfrak{p}_{\alpha, \beta}^{y} \geq-2 \sigma_{0},
$$

there exists $V_{y} \in H_{0}^{1}\left([\tilde{\alpha}, \tilde{\beta}], \mathbb{R}^{N}\right)$ with the following property:

for all $z \in H^{1}\left([\tilde{\alpha}, \tilde{\beta}], \mathbb{R}^{N}\right)$ with $\|z-y\|_{\tilde{\alpha}, \tilde{\beta}} \leq \rho_{0}$ it is:

(1) $V_{y}(s)=0$ for all $s \in[\tilde{\alpha}, \tilde{\beta}]$ such that $\phi(z(s)) \leq-\bar{\delta}+\varepsilon_{0}$;

(2) $g\left(\nabla \phi(z(s)), V_{y}(s)\right) \leq-\theta_{0}\left\|V_{y}\right\|_{\tilde{\alpha}, \tilde{\beta}}$, if $s \in[\tilde{\alpha}, \tilde{\beta}]$ and $\phi(z(s)) \in\left[-2 \sigma_{0}, 2 \sigma_{0}\right]$

(3) $\int_{\tilde{\alpha}}^{\tilde{\beta}} g\left(\dot{z}, \frac{\mathrm{D}}{\mathrm{d} t} V_{y}\right) d t \leq-\mu_{0}\left\|V_{y}\right\|_{\tilde{\alpha}, \tilde{\beta}}$.

Proof. We argue by contradiction, assuming the existence of sequences $\sigma_{n} \rightarrow 0^{+}\left(\sigma_{n}<\right.$ $\bar{\delta} / 2), \varepsilon_{n} \rightarrow 0^{+}, \varepsilon_{n}<\bar{\delta}-2 \sigma_{n}, \rho_{n} \rightarrow 0^{+}, \theta_{n} \rightarrow 0^{+}, \mu_{n} \rightarrow 0^{+}, y_{n} \in \mathfrak{M}_{0},\left[a_{n}, b_{n}\right] \in \mathcal{I}_{y_{n}}^{0}$ satisfying the property

$$
\frac{1}{2} \int_{a_{n}}^{b_{n}} g\left(\dot{y}_{n}, \dot{y}_{n}\right) d s \leq M_{0}
$$


and $\left[\alpha_{n}, \beta_{n}\right] \subset\left[a_{n}, b_{n}\right]$ such that:

$$
\left\{\begin{array}{l}
\phi\left(y\left(\alpha_{n}\right)\right)=\phi\left(y\left(\beta_{n}\right)\right)=-\bar{\delta}, \quad \phi\left(y_{n}(s)\right) \geq-\bar{\delta} \text { in }\left[\alpha_{n}, \beta_{n}\right] \\
\exists s_{n} \in\left[\alpha_{n}, \beta_{n}\right]: \phi\left(y_{n}\left(s_{n}\right)\right) \geq-\sigma_{n}, \\
\phi\left(y_{n}(s)\right) \leq 0, \quad \text { for all } s \in\left[\alpha_{n}, \beta_{n}\right]\left(\text { since }\left[\alpha_{n}, \beta_{n}\right] \subset\left[a_{n}, b_{n}\right] \in \mathcal{J}_{y_{n}}^{0}\right) \\
\max \left\{\left\|y_{n}\left(\alpha_{n}\right)-\pi\left(y_{n}\left(\beta_{n}\right)\right)\right\|_{E},\left\|\pi\left(y_{n}\left(\alpha_{n}\right)\right)-y_{n}\left(\beta_{n}\right)\right\|_{E}\right\} \geq \\
\quad(1+\bar{\gamma})\left\|\pi\left(y_{n}\left(\alpha_{n}\right)\right)-\pi\left(y_{n}\left(\beta_{n}\right)\right)\right\|_{E}
\end{array}\right.
$$

with $\left[\alpha_{n}, \beta_{n}\right]$ minimal with respect to the above properties and such that the following holds true:

for all $V \in H_{0}^{1}\left(\left[\alpha_{n}, \beta_{n}\right], \mathbb{R}^{N}\right)$, there exists $z=z\left(y_{n}, V\right)$ such that $\left\|z-y_{n}\right\|_{\alpha_{n}, \beta_{n}} \leq \rho_{n}$ and the conditions

(1) $V(s)=0$ for all $s \in\left[\alpha_{n}, \beta_{n}\right]$ such that $\phi(z(s)) \leq-\bar{\delta}+\varepsilon_{n}$;

(2) $g(\nabla \phi(z(s)), V(s)) \leq-\theta_{n}\|V\|_{\alpha_{n}, \beta_{n}}$, if $s \in\left[\alpha_{n}, \beta_{n}\right]$ and $\phi(z(s)) \in$ $\left[-2 \sigma_{n}, 2 \sigma_{n}\right]$

(3) $\int_{\alpha_{n}}^{\beta_{n}} g\left(\dot{z}, \frac{\mathrm{D}}{\mathrm{d} t} V\right) \mathrm{d} t \leq-\mu_{n}\|V\|_{\alpha_{n}, \beta_{n}}$,

do not hold true at the same time. (Note that $V$ can be consider equal to 0 outside $\left.\left[\alpha_{n}, \beta_{n}\right]\right)$.

In the following, we will first build a vector field $V$ satisfying (11)-(2) for every $z$ such that $\left\|z-y_{n}\right\|_{\alpha_{n}, \beta_{n}} \leq \rho_{n}$. Therefore, there must exist $\widehat{z}=\widehat{z}\left(y_{n}, V\right)$ such that property (3) does not hold, namely

$$
\int_{\alpha_{n}}^{\beta_{n}} g\left(\dot{\hat{z}}, \frac{\mathrm{D}}{\mathrm{d} t} V\right) \mathrm{d} t>-\mu_{n}\|V\|_{\alpha_{n}, \beta_{n}}
$$

Towards this goal first note that

$$
\int_{\alpha_{n}}^{\beta_{n}} g\left(\dot{y}_{n}, \dot{y}_{n}\right) \mathrm{d} s \leq \int_{a_{n}}^{b_{n}} g\left(\dot{y}_{n}, \dot{y}_{n}\right) \mathrm{d} s \leq 2 M_{0},
$$

while $\left|\beta_{n}-\alpha_{n}\right| \geq\left|\beta_{n}-s_{n}\right|$, which is bounded away from 0 because $\phi\left(x\left(\beta_{n}\right)\right)=-\bar{\delta}$ and $\phi\left(x\left(s_{n}\right)\right)=-\sigma_{n} \rightarrow 0$. Then up to subsequences there exists an interval $[\alpha, \beta] \subset[0,1]$ with $\alpha<\beta$ and a curve $y \in H^{1}\left([\alpha, \beta], \mathbb{R}^{N}\right)$ such that

$$
y_{n \mid\left[\alpha_{n}, \beta_{n}\right]} \rightarrow y_{\mid[\alpha, \beta]} \quad \text { weakly in } H^{1} \text { and uniformly. }
$$

By the properties satisfied by $y_{n \mid\left[\alpha_{n}, \beta_{n}\right]}$ and uniform convergence, we have

$$
\left\{\begin{array}{l}
\phi(y(\alpha))=\phi(y(\beta))=-\bar{\delta}, \phi(y(s)) \geq-\bar{\delta}, \forall s \in[\alpha, \beta] \\
\text { there exists } \left.s_{0} \in\right] \alpha, \beta\left[: \phi\left(y\left(s_{0}\right)\right)=0\right. \\
\phi(y(s)) \leq 0, \quad \forall s \in[\alpha, \beta], \\
\max \left\{\left\|y\left(s_{1}\right)-\pi\left(y\left(s_{2}\right)\right)\right\|_{E},\left\|\pi\left(y\left(s_{1}\right)\right)-y\left(s_{2}\right)\right\|_{E}\right\} \geq \\
\quad(1+\bar{\gamma})\left\|\pi\left(y\left(s_{1}\right)\right)-\pi\left(y\left(s_{2}\right)\right)\right\|_{E},
\end{array}\right.
$$

for all $s_{1} \in C_{\alpha}$ and all $s_{2} \in C_{\beta}$, where $C_{\alpha}$ (resp.: $C_{\beta}$ ) is the connected component of $(\phi \circ y)^{-1}(-\bar{\delta})$ containing $\alpha$ (resp.: $\beta$ ).

Take $\alpha^{\prime}=\sup \left\{s_{1}: s_{1} \in C_{\alpha}\right\}$ and $\beta^{\prime}=\inf \left\{s_{2}: s_{2} \in C_{\beta}\right\}$. Clearly $\phi(y(s))>-\bar{\delta}$ for all $s \in] \alpha^{\prime}, \beta^{\prime}\left[\right.$, while $\phi\left(y\left(\alpha^{\prime}\right)\right)=\phi\left(y\left(\beta^{\prime}\right)\right)=-\bar{\delta}$.

Now fix a sequence $V_{k} \in H_{0}^{1}\left([0,1], \mathbb{R}^{N}\right)$ such that (taking $k$ sufficiently large) $V_{k}(s)=$ 0 for all $s \notin\left[\alpha^{\prime}+\frac{1}{k}, \beta^{\prime}-\frac{1}{k}\right], V_{k} \not \equiv 0$ and $g\left(V_{k}(s), \nabla \phi(y(s))\right) \leq 0$ for all $s \in\left[\alpha^{\prime}, \beta^{\prime}\right]$ with $\phi(y(s))=0$. A simple contradiction argument shows that, for sufficiently large $n$, if $\left\|z-y_{n}\right\|_{\alpha_{n}, \beta_{n}} \leq \rho_{n}$, then $V=V_{k}$ satisfies property (1) with $[\alpha, \beta]=\left[\alpha_{n}, \beta_{n}\right]$, because $\varepsilon_{n} \rightarrow 0$ and $\phi(y(\delta))>0$ in $\left[\alpha^{\prime}+\frac{1}{k}, \beta^{\prime}-\frac{1}{k}\right]$. We can now modify $V_{k}$ so that also property (2) will be satisfied. 
To this aim, set:

$$
\begin{gathered}
\lambda_{n}=\sup \left\{\max \left\{\frac{g\left(V_{k}(s), \nabla \phi(w(s))\right)+\theta_{n}\left\|V_{k}\right\|_{\alpha_{n}, \beta_{n}}}{g\left(\nabla \phi\left(y_{n}(s)\right), \nabla \phi(w(s))\right)-\theta_{n}\left\|\nabla \phi\left(y_{n}\right)\right\|_{\alpha_{n}, \beta_{n}}}, 0\right\}:\right. \\
w \in H^{1}\left(\left[\alpha_{n}, \beta_{n}\right], \mathbb{R}^{N}\right),\left\|y_{n}-w\right\|_{\alpha_{n}, \beta_{n}} \leq \rho_{n}, \\
\left.s \in\left[\alpha_{n}, \beta_{n}\right], \phi(w(s)) \in\left[-2 \sigma_{n}, 2 \sigma_{n}\right]\right\} .
\end{gathered}
$$

The supremum above is finite for sufficiently large $n$, due to the facts that $\nabla \phi \neq 0$ in $\partial \Omega$, and that $\theta_{n} \rightarrow 0$ and $\left\|y_{n}\right\|_{\alpha_{n}, \beta_{n}}$ is bounded. Moreover, since $y_{n}$ is uniformly convergent to $y,\left\|y_{n}-w\right\|_{\left.L^{\infty}\left[\alpha_{n}, \beta_{n}\right], \mathbb{R}^{m}\right)} \rightarrow 0$ and $g\left(V_{k}(s), \nabla \phi(y(s))\right) \leq 0, \forall s \in[\alpha, \beta]$ such that $\phi(y(s))=0$, we see that

$$
\lim _{n \rightarrow \infty} \lambda_{n}=0 .
$$

For all $k$ sufficiently large, consider the piecewise affine function $\chi_{k}:[0,1] \rightarrow \mathbb{R}$ such that:

$$
\chi_{k}(s)= \begin{cases}0, & \text { if } s \leq \alpha^{\prime}+\frac{1}{k} \\ 1, & \text { if } s \in\left[\alpha^{\prime}+\frac{2}{k}, \beta^{\prime}-\frac{2}{k}\right] \\ 0 & \text { if } s \geq \beta^{\prime}-\frac{1}{k},\end{cases}
$$

and the vector field $V_{n, k} \in H_{0}^{1}\left(\left[\alpha_{n}, \beta_{n}\right], \mathbb{R}^{N}\right)$ :

$$
V_{n, k}(s)=V_{k}(s)-\lambda_{n} \chi_{k}(s) \nabla \phi\left(y_{n}(s)\right) \quad \text { for } s \in\left[\alpha_{n}, \beta_{n}\right] .
$$

Clearly, $\sup \left\|V_{n, k}\right\|_{\alpha_{n}, \beta_{n}}<+\infty$, and $V_{n, k} \not \equiv 0$ if $n$ is sufficiently large. Note that for $k$ and $n$ sufficiently large it is

$$
\phi\left(z_{n}(s)\right) \in\left[-2 \sigma_{n}, 2 \sigma_{n}\right] \quad \Rightarrow \quad s \in\left[\alpha^{\prime}+\frac{2}{k}, \beta^{\prime}-\frac{2}{k}\right],
$$

therefore (by the choice of $\lambda_{n}$ and $\chi_{k}$ ) $V_{n, k}$ satisfies property (2). Moreover, since $V_{k}$ satisfies (11) (for any $k$ sufficiently large) this is true also for $V_{n, k}$.

Then there exists $\hat{z}$ such that $\left\|\hat{z}-y_{n}\right\|_{\alpha_{n}, \beta_{n}} \leq \rho_{n} \rightarrow 0$ and

$$
\int_{\alpha_{n}}^{\beta_{n}} g\left(\dot{\hat{z}}, \frac{\mathrm{D}}{\mathrm{d} t} V_{n, k}\right) \mathrm{d} t>-\mu_{n}\left\|V_{n, k}\right\|_{\alpha_{n}, \beta_{n}}
$$

and since $\left\|V_{n, k}\right\|_{\alpha_{n}, \beta_{n}}$ is bounded,

$$
\liminf _{n \rightarrow+\infty} \int_{\alpha_{n}}^{\beta_{n}} g\left(\dot{\hat{z}}, \frac{\mathrm{D}}{\mathrm{d} t} V_{n, k}\right) \mathrm{d} t \geq 0
$$

Now, $\left\|\hat{z}-y_{n}\right\|_{\alpha_{n}, \beta_{n}} \rightarrow 0$, then we obtain

$$
\liminf _{n \rightarrow \infty} \int_{\alpha_{n}}^{\beta_{n}} g\left(\dot{y}_{n}, \frac{\mathrm{D}}{\mathrm{d} t} V_{n, k}\right) \mathrm{d} t \geq 0
$$

and in addition $\lambda_{n} \rightarrow 0$, while $\nabla \phi\left(y_{n}\right)$ is bounded in $H^{1}\left(\left[\alpha_{n}, \beta_{n}\right], \mathbb{R}^{N}\right)$; therefore $V_{n, k}$ tends to $V_{k}$ in $H^{1}\left(\left[\alpha_{n}, \beta_{n}\right], \mathbb{R}^{N}\right)$, so

$$
\liminf _{n \rightarrow \infty} \int_{\alpha_{n}}^{\beta_{n}} g\left(\dot{y}_{n}, \frac{\mathrm{D}}{\mathrm{d} t} V_{k}\right) \mathrm{d} t \geq 0 .
$$

Moreover $V_{k}=0$ outside $\left[\alpha^{\prime}+\frac{1}{k}, \beta^{\prime}-\frac{1}{k}\right]$ and $\left[\alpha_{n}, \beta_{n}\right] \supset\left[\alpha^{\prime}+\frac{1}{k}, \beta^{\prime}-\frac{1}{k}\right]$. Therefore

$$
\liminf _{n \rightarrow \infty} \int_{\alpha^{\prime}+1 / k}^{\beta^{\prime}-1 / k} g\left(\dot{y}_{n}, \frac{\mathrm{D}}{\mathrm{d} t} V_{k}\right) \mathrm{d} t \geq 0
$$


and, since $\dot{y}_{n} \rightarrow \dot{y}$ in $L^{2}\left(\left[\alpha^{\prime}+\frac{1}{k}, \beta^{\prime}-\frac{1}{k}\right], \mathbb{R}^{N}\right)$, we get

$$
0 \leq \lim _{n \rightarrow \infty} \int_{\alpha^{\prime}+1 / k}^{\beta^{\prime}-1 / k} g\left(\dot{y}_{n}, \frac{\mathrm{D}}{\mathrm{d} t} V_{k}\right) \mathrm{d} t=\int_{\alpha^{\prime}+1 / k}^{\beta^{\prime}-1 / k} g\left(\dot{y}, \frac{\mathrm{D}}{\mathrm{d} t} V_{k}\right) \mathrm{d} t=\int_{\alpha^{\prime}}^{\beta^{\prime}} g\left(\dot{y}, \frac{\mathrm{D}}{\mathrm{d} t} V_{k}\right) \mathrm{d} t
$$

because $V_{k}=0$ on $\left[\alpha^{\prime}, \alpha^{\prime}+\frac{1}{k}\right] \cup\left[\beta^{\prime}-\frac{1}{k}, \beta^{\prime}\right]$. Finally, by arbitrariness of the interval $\left[\alpha^{\prime}+\frac{1}{k}, \beta^{\prime}-\frac{1}{k}\right]$, we have

$$
\int_{\alpha^{\prime}}^{\beta^{\prime}} g\left(\dot{y}, \frac{\mathrm{D}}{\mathrm{d} t} V\right) \mathrm{d} t \geq 0
$$

for all $V \in H_{0}^{1}\left(\left[\alpha^{\prime}, \beta^{\prime}\right], \mathbb{R}^{N}\right)$ such that

$$
g(V(s), \nabla \phi(y(s))) \leq 0 \text { for all } s \text { satisfying } \phi(y(s))=0 .
$$

But this is contradiction with (7.12) and Lemma 7.2, and the proof is complete.

Remark 7.7. To obtain flows moving far from curves having topologically non essential intervals (that we define below) it is crucial to fix a constant $\left.\left.\sigma_{1} \in\right] 0, \sigma_{0}\right]$ such that

$$
\sigma_{1} \leq \frac{2}{7} \rho_{0} \theta_{0},
$$

where $\rho_{0}, \theta_{0}$ are given by Proposition 7.6.

Proposition 7.6 is a crucial ingredient to define the class of the admissible homotopies, whose elements will avoid irregular variationally critical points of first type. The description of this class is based of the notion of topologically non essential interval given below.

Let $\bar{\delta}$ be as in (7.8), $\bar{\gamma}$ as in Lemma 7.1 and $\sigma_{1}$ as in Remark 7.7.

Definition 7.8. Let $y \in \mathfrak{M}$ be fixed. An interval $[\alpha, \beta] \subset[a, b] \in \mathcal{I}_{y}^{0}$, is called topologically not essential interval (for $y$ ) if $y$ is $\bar{\delta}$-close to $\partial \Omega$ on $[\alpha, \beta]$, with $\mathfrak{p}_{\alpha, \beta}^{y} \geq-\sigma_{1}$ and $\mathfrak{b}_{\alpha, \beta}^{y} \geq\left(1+\frac{3}{2} \bar{\gamma}\right)$.

Remark 7.9. By Lemma 7.1 the intervals $[\alpha, \beta]$ containing cusp intervals $\left[t_{1}, t_{2}\right]$ of curves $x$, which are irregular variationally critical portion of first type, and satisfying $\Theta_{x}\left(t_{1}, t_{2}\right) \geq$ $d_{0}$ are topologically not essential intervals with $\mathfrak{p}_{\alpha, \beta}^{x}=0$ and $\mathfrak{b}_{\alpha, \beta}^{x} \geq 1+2 \bar{\gamma}$. This fact will allow us to use Proposition 11.1 in section 11 and move away from the set of irregular variationally critical portions of first type without increasing the value of the energy functional.

\section{THE ADMISSIBLE HOMOTOPIES}

In the present section we shall list the properties of the admissible homotopies used to prove existence result. This choice is clearly crucial for the proof: the notion of topological critical level, that we shall use also in this paper to obtain the existence result, depends indeed by the choice of the admissible homotopies.

We shall consider continuous homotopies $h:[0,1] \times \mathcal{D} \rightarrow \mathfrak{M}$ where $\mathcal{D}$ is a closed $\mathcal{R}$-invariant subset of $\mathfrak{C}$. (However the following notions can be done at the same way when $h$ is defined in any $\mathcal{R}$-invariant subset of $\mathfrak{M}$, not necessarily included in $\mathfrak{C}$ ).

Recall that $\mathfrak{C}$ and $\mathfrak{C}_{0}$ are defined in (2.6). The following basic properties for our admissible homotopies holds:

$$
h(\tau, \gamma) \text { is a constant curve for all } \tau \in[0,1] \text {, for all } \gamma \in \mathfrak{C}_{0} \cap \mathcal{D}
$$

$h$ is $\mathcal{R}$-equivariant, namely $h(\tau, \mathcal{R} \gamma)=\mathcal{R} h(\tau, \gamma)$ for all $\tau \in[0,1], \gamma \in \mathcal{D}$. 
The homotopies that we shall use are of 3 types: outgoing homotopies, reparameterizazions and ingoing homotpies. They can be described in the following way.

Definition 8.1. Let $0 \leq \tau^{\prime}<\tau^{\prime \prime} \leq 1$. We say that $h$ is of type $A$ in $\left[\tau^{\prime}, \tau^{\prime \prime}\right]$ if it satisfies the following properties for all $\tau_{0} \in\left[\tau^{\prime}, \tau^{\prime \prime}\right]$, for all $s_{0} \in[0,1]$, for all $x \in \mathcal{D}$ :

(1) if $\phi\left(h\left(\tau_{0}, x\right)\left(s_{0}\right)\right)=0$, then $\tau \mapsto \phi\left(h(\tau, x)\left(s_{0}\right)\right)$ is strictly increasing in a neighborhood of $\tau_{0}$.

(2) for all $\left.\delta \in] 0, \delta_{0}\right]$, if $\left[\alpha_{\tau_{0}}, \beta_{\tau_{0}}\right]$ is a $\delta$-interval for $h\left(\tau_{0}, x\right), s \in\left[\alpha_{\tau_{0}}, \beta_{\tau_{0}}\right]$ and $\phi\left(h\left(\tau_{0}, x\right)\left(s_{0}\right)\right)=-\delta$, then $\tau \mapsto \phi\left(h(\tau, x)\left(s_{0}\right)\right)$ is strictly increasing in a neighborhood of $\tau_{0}$.

Remark 8.2. It is relevant to observe that, by property above of Definition 8.1 , if $\left[a_{\tau}, b_{\tau}\right]$ denotes any interval in $\mathcal{I}_{h(\tau, \gamma)}$ we have:

$$
\tau^{\prime} \leq \tau_{1}<\tau_{2} \leq \tau^{\prime \prime} \text { and }\left[a_{\tau_{1}}, b_{\tau_{1}}\right] \cap\left[a_{\tau_{2}}, b_{\tau_{2}}\right] \neq \emptyset \Longrightarrow\left[a_{\tau_{2}}, b_{\tau_{2}}\right] \subset\left[a_{\tau_{1}}, b_{\tau_{1}}\right] .
$$

Definition 8.3. Let $0 \leq \tau^{\prime}<\tau^{\prime \prime} \leq 1$. We say that $h$ is of type $B$ in $\left[\tau^{\prime}, \tau^{\prime \prime}\right]$ if it satisfies the following property:

$$
\begin{aligned}
& \exists \Lambda:\left[\tau^{\prime}, \tau^{\prime \prime}\right] \times \mathcal{H}_{0}^{1}([0,1],[0,1]) \rightarrow[0,1] \text { continuous and such that } \\
& \Lambda(\tau, \gamma)(0)=0, \Lambda(\tau, \gamma)(1)=1, \forall \tau \in\left[\tau^{\prime}, \tau^{\prime \prime}\right], \forall \gamma \in \mathcal{D} \\
& s \mapsto \Lambda(\tau, \gamma)(s) \text { is strictly increasing in }[0,1], \forall \tau \in\left[\tau^{\prime}, \tau^{\prime \prime}\right], \forall \gamma \in \mathcal{D} \text {, } \\
& \Lambda(0, \gamma)(s)=s \text { for any } \gamma \in \mathcal{D}, s \in[0,1] \text { and } \\
& h(\tau, \gamma)(s)=(\gamma \circ \Lambda(\tau, \gamma))(s) \forall \tau \in\left[\tau^{\prime}, \tau^{\prime \prime}\right], \forall s \in[0,1], \forall \gamma \in \mathcal{D} .
\end{aligned}
$$

Definition 8.4. Let $0 \leq \tau^{\prime}<\tau^{\prime \prime} \leq 1$. We say that $h$ is of type $C$ in $\left[\tau^{\prime}, \tau^{\prime \prime}\right]$ if it satisfies the following properties:

(1) $h\left(\tau^{\prime}, \gamma\right)(s) \neq \bar{\Omega} \Rightarrow h(\tau, \gamma)(s)=h\left(\tau^{\prime}, \gamma\right)(s)$ for any $\tau \in\left[\tau^{\prime}, \tau^{\prime \prime}\right]$;

(2) $h\left(\tau^{\prime}, \gamma\right)(s) \in \Omega \Rightarrow h(\tau, \gamma)(s) \in \Omega$ for any $\tau \in\left[\tau^{\prime}, \tau^{\prime \prime}\right]$;

The interval $[0,1]$ where $\tau$ lives will be partitioned in the following way:

There exists a partition of the interval $[0,1], 0=\tau_{0}<\tau_{1}<\ldots<\tau_{k}=1$ such that on any interval $\left[\tau_{i}, \tau_{i+1}\right], i=0, \ldots, k-1$, the homotopy $h$ is of type A,B, or C.

Moreover, in order to move far from topologically not essential intervals (cf. Definition 7.8) we need the following further property:

(8.5) for any $[a, b] \in \mathcal{J}_{h(\tau, \gamma)}$ and for all $[\alpha, \beta] \subset[a, b]$ topologically non essential,

$$
\phi(h(\tau, \gamma)(s)) \leq-\frac{\sigma_{1}}{2} \text { for all } s \in[\alpha, \beta]
$$

where $\sigma_{1}$ is defined in Remark 7.7.

We finally define the following classes of admissible homotopies:

$$
\mathcal{H}=\{(\mathcal{D}, h): \mathcal{D} \text { is closed, } \mathcal{R} \text {-invariant subset of } \mathfrak{C} \text { and }
$$

$$
h:[0,1] \times \mathcal{D} \rightarrow \mathfrak{M} \text { satisfies } 8.1)-(8.4)\},
$$

$$
\left.\mathcal{H}_{1}=\{(\mathcal{D}, h) \in \mathcal{H} \text { such that } h:[0,1] \times \mathcal{D} \rightarrow \mathfrak{M} \text { satisfies also } 8.5)\right\} .
$$

Remark 8.5. Observe that, thanks to the properties of $\mathfrak{C}$, denoting by $h_{0}$ the constant identity homotopy it is $\left(\mathfrak{C}, h_{0}\right) \in \mathcal{H}_{1}$. 
Homotopies of type A will be used far from variationally critical portions, homotopies of type B nearby variationally critical portions of II type, while homotopies of type $\mathrm{C}$ will be used nearby variationally critical portions of I type.

We shall use the following functional defined for any $(\mathcal{D}, h) \in \mathcal{H}$ :

$$
\mathcal{F}(\mathcal{D}, h)=\sup \left\{\frac{b-a}{2} \int_{a}^{b} g(\dot{y}, \dot{y}) \mathrm{d} s: y=h(1, x), x \in \mathcal{D},[a, b] \in \mathcal{I}_{y}\right\} .
$$

Remark 8.6. It is to pointed out that any $\frac{(b-a)}{2} \int_{a}^{b} g(\dot{y}, \dot{y}) \mathrm{d} t$ coincides with the integral $\frac{1}{2} \int_{0}^{1} g\left(\dot{y}_{a, b}, \dot{y}_{a, b}\right) \mathrm{d} t$, where $y_{a, b}$ is the affine reparameterization of $y$ on the interval $[0,1]$.

Given continuous maps $h_{1}:[0,1] \times F_{1} \rightarrow \mathfrak{M}$ and $h_{2}:[0,1] \times F_{2} \rightarrow \mathfrak{M}$ such that $h_{1}\left(1, F_{1}\right) \subset F_{2}$, then we define the concatenation of $h_{1}$ and $h_{2}$ as the continuous map $h_{2} \star h_{1}:[0,1] \times F_{1} \rightarrow \Lambda$ given by

$$
h_{2} \star h_{1}(t, x)= \begin{cases}h_{1}(2 t, x), & \text { if } t \in\left[0, \frac{1}{2}\right], \\ h_{2}\left(2 t-1, h_{1}(1, x)\right), & \text { if } t \in\left[\frac{1}{2}, 1\right] .\end{cases}
$$

\section{A DEFormation lemma With homotopies of type A}

In this section we deal with homotopy of type A. From a technical point of view this is the more difficult case. For this reason our approach to deformation lemmas starts with the function al $\mathcal{F}$ defined just on the class $\mathcal{H}$, instead of $\mathcal{H}_{1}$.

The proof of Proposition 9.1 below is based on the construction of integral flows of vector fields in $\mathcal{V}^{-}$, that can be carried on thanks to the results of Propositions 6.1 and 6.4 .

It is important to point out that among the energy integrals used to define the functional $\mathcal{F}$ it is possible to have some energy integral that is not decreasing along the flow. This is the reason of the use of Lemma 9.2. It will allow us to obtain a global flow where $\mathcal{F}$ realizes a smaller value.

Now for all $[a, b] \subset[0,1]$ let $\mathcal{Z}_{a, b}$ be as in (6.1).

Proposition 9.1. Fix $c_{1} \in\left[\frac{1}{2}\left(\frac{3 \delta_{0}}{4 K_{0}}\right)^{2}, M_{0}\left[\right.\right.$ and $r>0$. Then, there exists $\varepsilon_{1}=\varepsilon_{1}\left(r, c_{1}\right) \in$ ] $0, \frac{c_{1}}{4}[$ such that for any $\left.\varepsilon \in] 0, \varepsilon_{1}\right]$, for any $(\mathcal{D}, h) \in \mathcal{H}$ satisfying:

$$
\mathcal{F}(\mathcal{D}, h) \leq c_{1}+\varepsilon
$$

and

$$
\begin{aligned}
& \left\|y-x_{\mid[a, b]}\right\|_{a, b} \geq r \\
& \qquad \text { for any } y \in \mathcal{Z}_{a, b}, \text { for any } x=h(1, \xi), \xi \in \mathcal{D} \text {, such that } \\
& \qquad \frac{b-a}{2} \int_{a}^{b} g(\dot{x}, \dot{x}) \mathrm{d} s \geq c_{1}-\varepsilon \text {, where }[a, b] \in \mathcal{I}_{x} .
\end{aligned}
$$

there exists a continuous map $H_{\varepsilon}:[0,1] \times h(1, \mathcal{D}) \rightarrow \mathfrak{M}$ of type $A$ in $[0,1]$ with the following properties:

(1) $\left(\mathcal{D}, H_{\varepsilon} \star h\right) \in \mathcal{H}$;

(2) $\mathcal{F}\left(\mathcal{D}, H_{\varepsilon} \star h\right) \leq c_{1}-\varepsilon$;

(3) there exists $T_{\varepsilon}>0$, with $T_{\varepsilon} \rightarrow 0$ as $\varepsilon \rightarrow 0$, such that for any $z \in h(1, \mathcal{D})$ $\left\|H_{\varepsilon}(\tau, z)-z\right\|_{a, b} \leq \tau T_{\varepsilon}$ for all $\tau \in[0,1]$, for all $[a, b] \in \mathcal{I}_{z}$.

For the proof of Proposition 9.1 some preliminary results are needed. To state the first Lemma observe that, because of the compactness $\left.\left.\phi^{-1}(]-\infty, \delta_{0}\right]\right)$, analogously to (6.14), there exists constant $K_{1}, K_{2}, K_{3} \in \mathbb{R}^{+}$such that

$$
\begin{aligned}
& \left|g_{x}\left(v_{1}, v_{1}\right)-g_{z}\left(v_{2}, v_{2}\right)\right| \leq K_{1}\|x-z\|_{E}\left\|v_{1}\right\|_{E}^{2}+K_{2}\left\|v_{1}-v_{2}\right\|_{E}\left\|v_{1}\right\|_{E} \\
& \left.\left.\quad+K_{3}\left\|v_{1}-v_{2}\right\|_{E}\left\|v_{2}\right\|_{E} \text { for any } x, z \in \phi^{-1}(]-\infty, \delta_{0}\right]\right) \text { and } v_{1}, v_{2} \in \mathbb{R}^{N}
\end{aligned}
$$


Set

$$
\widehat{K}=\frac{K_{1}}{\ell_{0}}\left(M_{0}+1\right)+\frac{K_{2}}{\sqrt{\ell_{0}}} \sqrt{M_{0}+1}+\frac{K_{3}}{\sqrt{\ell_{0}}} \sqrt{M_{0}},
$$

where $\ell_{0}$ is given in 6.13). We have

Lemma 9.2. Fix $K_{*}>0$ and $\rho_{*}=\frac{c_{1} K_{*}}{32 M_{0} \widehat{K}}$. Fix $x \in \mathfrak{M}$ and $\left[a_{x}, b_{x}\right] \in \mathcal{I}_{x}$ (and therefore $\left.\int_{a_{x}}^{b_{x}} \frac{1}{2} g(\dot{x}, \dot{x}) d s<M_{0}\right)$. Take $\left[\alpha_{x}, \beta_{x}\right] \supset\left[a_{x}, b_{x}\right]$ and included in $[0,1]$ such that:

$$
\begin{gathered}
\int_{\alpha_{x}}^{a_{x}} \frac{1}{2} g(\dot{x}, \dot{x}) d s \leq \frac{K_{*}}{4}, \int_{b_{x}}^{\beta_{x}} \frac{1}{2} g(\dot{x}, \dot{x}) d s \leq \frac{K_{*}}{4}, \int_{\alpha_{x}}^{\beta_{x}} \frac{1}{2} g(\dot{x}, \dot{x}) d s \leq M_{0}+1 \\
\left(a_{x}-\alpha_{x}\right)\left(M_{0}+\frac{K_{*}}{4}\right) \leq \frac{c_{1} K_{*}}{16 M_{0}},\left(\beta_{x}-b_{x}\right)\left(M_{0}+\frac{K_{*}}{4}\right) \leq \frac{c_{1} K_{*}}{16 M_{0}} .
\end{gathered}
$$

Take $z \in \mathfrak{M}$ and $\left[a_{z}, b_{z}\right]$ satisfying:

$$
\begin{gathered}
{\left[a_{z}, b_{z}\right] \subset\left[\alpha_{x}, \beta_{x}\right] \text { and }\left[a_{z}, b_{z}\right] \cap\left[a_{x}, b_{x}\right] \neq \emptyset,} \\
D\left(x, a_{z}, b_{z}, a_{x}, b_{x}\right)>K_{*}(c f .6 .60), \\
\|z-x\|_{a_{z}, b_{z}}<\rho_{*},
\end{gathered}
$$

(so by (1.9) we have also $\|z-x\|_{L^{\infty}\left(\left[a_{z}, b_{z}\right], \mathbb{R}^{N}\right)}<\rho_{*}$ ).

Then the following property is satisfied:

$$
\frac{1}{2} \int_{a_{z}}^{b_{z}} g(\dot{z}, \dot{z}) \mathrm{d} s \leq \frac{1}{2} \int_{a_{x}}^{b_{x}} g(\dot{x}, \dot{x}) \mathrm{d} s-\frac{K_{*}}{8} .
$$

Moreover if

$$
\left(b_{x}-a_{x}\right) \int_{a_{x}}^{b_{x}} \frac{1}{2} g(\dot{x}, \dot{x}) d s \geq \frac{c_{1}}{2}
$$

we also have

$$
\frac{b_{z}-a_{z}}{2} \int_{a_{z}}^{b_{z}} g(\dot{z}, \dot{z}) \mathrm{d} s \leq \frac{b_{x}-a_{x}}{2} \int_{a_{x}}^{b_{x}} g(\dot{x}, \dot{x}) \mathrm{d} s-\frac{c_{1} K_{*}}{32 M_{0}} .
$$

Proof. By (6.13), 9.4), assumption (9.9) and Hölder inequality

$$
\begin{aligned}
&\left|\frac{1}{2} \int_{a_{z}}^{b_{z}} g(\dot{x}, \dot{x}) \mathrm{d} s-\frac{1}{2} \int_{a_{z}}^{b_{z}} g(\dot{z}, \dot{z}) \mathrm{d} s\right| \\
& K_{1} \rho_{*} \int_{a_{z}}^{b_{z}}\|\dot{x}\|_{E}^{2}+K_{2} \rho_{*} \sqrt{\int_{a_{z}}^{b_{z}}\|\dot{x}\|_{E}^{2}}+K_{3} \rho_{*} \sqrt{\int_{a_{z}}^{b_{z}}\|\dot{z}\|_{E}^{2}} \leq \\
& \frac{K_{1} \rho_{*}}{\ell_{0}} \int_{a_{z}}^{b_{z}} g(\dot{x}, \dot{x})+\frac{K_{2} \rho_{*}}{\sqrt{\ell_{0}}} \sqrt{\int_{a_{z}}^{b_{z}} g(\dot{x}, \dot{x})}+\frac{K_{3} \rho_{*}}{\sqrt{\ell_{0}}} \sqrt{\int_{a_{z}}^{b_{z}} g(\dot{z}, \dot{z}) .}
\end{aligned}
$$

But $\left[a_{z}, b_{z}\right] \subset\left[\alpha_{x}, \beta_{x}\right]$ and $\left[a_{z}, b_{z}\right] \in \mathcal{I}_{z}$. Therefore by (9.5) and (9.4),

$$
\left|\frac{1}{2} \int_{a_{z}}^{b_{z}} g(\dot{x}, \dot{x}) \mathrm{d} s-\frac{1}{2} \int_{a_{z}}^{b_{z}} g(\dot{z}, \dot{z}) \mathrm{d} s\right| \leq \widehat{K} \rho_{*},
$$

from which we deduce

$$
\frac{1}{2} \int_{a_{z}}^{b_{z}} g(\dot{z}, \dot{z}) \mathrm{d} s \leq \frac{1}{2} \int_{a_{z}}^{b_{z}} g(\dot{x}, \dot{x}) \mathrm{d} s+\widehat{K} \rho_{*} .
$$


Now assumption 9.8 says that

$$
\frac{1}{2} \int_{I_{a_{x}, a_{z}} \cup I_{b_{z}, b_{x}}} g(\dot{x}, \dot{x}) \mathrm{d} s>K_{*}
$$

where $I_{a, b}$ denotes the interval $[a, b]$ if $b \geq a$ and the interval $[b, a]$ if $b<a$. Just to fix our ideas we can assume

$$
\frac{1}{2} \int_{I_{a_{x}, a_{z}}} g(\dot{x}, \dot{x}) \mathrm{d} s>\frac{K_{*}}{2} .
$$

In this case, thanks to 9.5 and $(9.7)$ we deduce that $a_{z} \in\left[a_{x}, b_{x}\right]$. Then

$$
\begin{aligned}
\frac{1}{2} \int_{a_{z}}^{b_{z}} g(\dot{x}, \dot{x}) \mathrm{d} s=\frac{1}{2} \int_{a_{x}}^{b_{x}} g(\dot{x}, \dot{x}) \mathrm{d} s+ & \\
& -\frac{1}{2} \int_{a_{x}}^{a_{z}} g(\dot{x}, \dot{x}) \mathrm{d} s+\frac{1}{2} \int_{b_{x}}^{b_{z}} g(\dot{x}, \dot{x}) \mathrm{d} s \text { if } b_{z} \geq b_{x}
\end{aligned}
$$

and

$$
\begin{aligned}
\frac{1}{2} \int_{a_{z}}^{b_{z}} g(\dot{x}, \dot{x}) \mathrm{d} s=\frac{1}{2} \int_{a_{x}}^{b_{x}} g(\dot{x}, & \dot{x}) \mathrm{d} s+ \\
& -\frac{1}{2} \int_{a_{x}}^{a_{z}} g(\dot{x}, \dot{x}) \mathrm{d} s-\frac{1}{2} \int_{b_{z}}^{b_{x}} g(\dot{x}, \dot{x}) \mathrm{d} s \text { if } b_{z}<b_{x} .
\end{aligned}
$$

Then

(9.16) $\frac{1}{2} \int_{a_{z}}^{b_{z}} g(\dot{x}, \dot{x}) \mathrm{d} s \leq \frac{1}{2} \int_{a_{x}}^{b_{x}} g(\dot{x}, \dot{x}) \mathrm{d} s-\frac{1}{2} \int_{I_{a_{x}, a_{z}}} g(\dot{x}, \dot{x}) \mathrm{d} s+\frac{1}{2} \int_{b_{x}}^{\beta_{x}} g(\dot{x}, \dot{x}) \mathrm{d} s$, so combining $9.14-(9.16$ ) and using assumptions 9.5 and $(9.6$ gives

$$
\frac{1}{2} \int_{a_{z}}^{b_{z}} g(\dot{z}, \dot{z}) \mathrm{d} s \leq \frac{1}{2} \int_{a_{x}}^{b_{x}} g(\dot{x}, \dot{x}) \mathrm{d} s-\frac{K_{*}}{4}+\widehat{K} \rho_{*}
$$

obtaining $(9.10)$ because of the choice of $\rho_{*}$ (recall that $c_{1}<M_{0}$.)

In order to prove 9.12 first observe that, by (9.14),

$$
\left(b_{z}-a_{z}\right) \frac{1}{2} \int_{a_{z}}^{b_{z}} g(\dot{z}, \dot{z}) \mathrm{d} s \leq\left(b_{z}-a_{z}\right) \frac{1}{2} \int_{a_{z}}^{b_{z}} g(\dot{x}, \dot{x}) \mathrm{d} s+\left(b_{z}-a_{z}\right) \widehat{K} \rho_{*} .
$$

Moreover recall that by assumptions 9.5 ) and (9.7), since we are assuming $(9.15)$, we have

$$
a_{z} \in\left[a_{x}, b_{x}\right] .
$$

Then if $b_{z} \leq b_{x}$ we have $b_{z}-a_{z} \leq b_{x}-a_{x}$, so by (9.17),

$$
\left(b_{z}-a_{z}\right) \frac{1}{2} \int_{a_{z}}^{b_{z}} g(\dot{z}, \dot{z}) \mathrm{d} s \leq\left(b_{x}-a_{x}\right) \frac{1}{2} \int_{a_{z}}^{b_{z}} g(\dot{x}, \dot{x}) \mathrm{d} s+\left(b_{x}-a_{x}\right) \widehat{K} \rho_{*},
$$

therefore by $(9.5)$ and 9.16

$$
\left(b_{z}-a_{z}\right) \frac{1}{2} \int_{a_{z}}^{b_{z}} g(\dot{z}, \dot{z}) \mathrm{d} s \leq\left(b_{x}-a_{x}\right) \frac{1}{2} \int_{a_{x}}^{b_{x}} g(\dot{x}, \dot{x}) \mathrm{d} s+\left(b_{x}-a_{x}\right)\left(-\frac{K_{*}}{2}+\frac{K_{*}}{4}+\widehat{K} \rho_{*}\right),
$$

from which we deduce 9.12 thanks to the choice of $\rho_{*}$ and property 9.11 that implies $b_{x}-a_{x} \geq \frac{c_{1}}{2 M_{0}}$. 
Now assume $b_{z}>b_{x}$. In this case we have

$$
\begin{array}{r}
\left(b_{z}-a_{z}\right) \frac{1}{2} \int_{a_{z}}^{b_{z}} g(\dot{x}, \dot{x}) \mathrm{d} s=\left(b_{z}-b_{x}\right) \frac{1}{2} \int_{a_{z}}^{b_{z}} g(\dot{x}, \dot{x}) \mathrm{d} s+\left(b_{x}-a_{z}\right) \frac{1}{2} \int_{a_{z}}^{b_{z}} g(\dot{x}, \dot{x}) \mathrm{d} s \leq \\
\left(\beta_{x}-b_{x}\right)\left(\frac{1}{2} \int_{a_{x}}^{b_{x}} g(\dot{x}, \dot{x}) \mathrm{d} s+\frac{1}{2} \int_{b_{x}}^{\beta_{x}} g(\dot{x}, \dot{x}) \mathrm{d} s\right)+\left(b_{x}-a_{x}\right) \frac{1}{2} \int_{a_{z}}^{b_{z}} g(\dot{x}, \dot{x}) \mathrm{d} s \leq \\
\frac{c_{1} K_{*}}{16 M_{0}}+\left(b_{x}-a_{x}\right) \frac{1}{2} \int_{a_{z}}^{b_{z}} g(\dot{x}, \dot{x}) \mathrm{d} s
\end{array}
$$

by $(9.5)$ and $(9.6)$.

Then by 9.17 we have

$$
\left(b_{z}-a_{z}\right) \frac{1}{2} \int_{a_{z}}^{b_{z}} g(\dot{z}, \dot{z}) \mathrm{d} s \leq \frac{c_{1} K_{*}}{16 M_{0}}+\left(b_{x}-a_{x}\right) \frac{1}{2} \int_{a_{z}}^{b_{z}} g(\dot{x}, \dot{x}) \mathrm{d} s+\widehat{K} \rho_{*},
$$

so by 9.15 , , 9.16) and assumption (9.5),

$$
\left(b_{z}-a_{z}\right) \frac{1}{2} \int_{a_{z}}^{b_{z}} g(\dot{z}, \dot{z}) \mathrm{d} s \leq \frac{c_{1} K_{*}}{4 M_{0}}+\widehat{K} \rho_{*}+\left(b_{x}-a_{x}\right)\left(\frac{1}{2} \int_{a_{x}}^{b_{x}} g(\dot{x}, \dot{x}) \mathrm{d} s-\frac{K_{*}}{4}\right) \text {. }
$$

Therefore, recalling that $b_{x}-a_{x} \geq \frac{c_{1}}{2 M_{0}}$, it is

$$
\left(b_{z}-a_{z}\right) \frac{1}{2} \int_{a_{z}}^{b_{z}} g(\dot{z}, \dot{z}) \mathrm{d} s \leq \frac{c_{1} K_{*}}{16 M_{0}}+\widehat{K} \rho_{*}-\frac{c_{1} K_{*}}{8 M_{0}}+\left(b_{x}-a_{x}\right) \frac{1}{2} \int_{a_{x}}^{b_{x}} g(\dot{x}, \dot{x}) \mathrm{d} s
$$

giving 9.12) because of the choice of $\rho_{*}$.

Remark 9.3. In the proof of Proposition 9.1 we shall use the above Lemma with $K_{*}=$ $E(r)$, in order to have that conditions 9.10 and 9.12 are satisfies if

$$
D\left(x, a_{z}, b_{z}, a_{x}, b_{x}\right)>E(r) .
$$

In this case it may happen that $\frac{1}{2} \int_{a_{z}}^{b_{z}} g(\dot{z}, \dot{z}) \mathrm{d} s$ and $\left(b_{z}-a_{z}\right) \frac{1}{2} \int_{a_{z}}^{b_{z}} g(\dot{z}, \dot{z}) \mathrm{d} s$ are not decreasing along the flow that we shall construct by means of the vector filed $V_{x}$ given by Proposition 6.4. But condition (9.10) says that $\frac{1}{2} \int_{a_{z}}^{b_{z}} g(\dot{z}, \dot{z}) \mathrm{d} s$ is much less than $M_{0}$, while condition 9.12) says that $\left(b_{z}-a_{z}\right) \frac{1}{2} \int_{a_{z}}^{b_{z}} g(\dot{z}, \dot{z}) \mathrm{d} s$ is much less then $\left(b_{x}-\right.$ $\left.a_{x}\right) \frac{1}{2} \int_{a_{x}}^{b_{x}} g(\dot{x}, \dot{x}) \mathrm{d} s$, and this will be useful to get a flow in the space $\mathfrak{M}$ where $\mathcal{F}$ reaches smaller values.

Definition 9.4. Fix $\tau_{0} \in[0, \delta]$. The set

$$
\mathcal{C}_{\tau_{0}, \delta}=\left\{\left[a_{\tau}, b_{\tau}\right] \subset[0,1]: \tau_{0} \leq \tau_{1} \leq \tau_{2} \leq \delta \Longrightarrow\left[a_{\tau_{2}}, b_{\tau_{2}}\right] \subset\left[a_{\tau_{1}}, b_{\tau_{1}}\right]\right\}
$$

is called non increasing intervals collection (NIIC).

Lemma 9.5. Let $W$ be a locally Lipschitz continuous vector field in $H^{1}\left([0,1], \mathbb{R}^{N}\right), \delta>0$ and $\eta_{z}:[0, \delta] \rightarrow \mathfrak{M}_{0}$ be the solution of the Cauchy problem

$$
\left\{\begin{array}{l}
\frac{\mathrm{d}}{\mathrm{d} \tau} \eta_{z}=W\left(\eta_{z}\right) \\
\eta_{z}(0)=z, z \in \mathfrak{M}_{0}
\end{array}\right.
$$

Fix $\tau_{0} \in[0, \delta]$ and a NIIC $\mathcal{C}_{\tau_{0}, \delta}=\left\{\left[a_{\tau}, b_{\tau}\right]: \tau \in\left[\tau_{0}, \delta\right]\right\}$ such that

$$
\int_{a_{\tau}}^{b_{\tau}} \frac{1}{2} g\left(\dot{\eta}_{z}(\tau), \dot{\eta}_{z}(\tau)\right) d s<M_{0}, \text { for any }\left[a_{\tau}, b_{\tau}\right] \in C_{\tau_{0}, \delta}
$$

where $\dot{\eta}_{z}(\tau)$ denotes the derivative with respect to $\tau$ of the curve $\eta_{z}(\tau)$, and

$$
\left\|W\left(\eta_{z}(\tau)\right)\right\|_{a_{\tau}, b_{\tau}} \leq 1 \text { for any } \tau \in\left[\tau_{0}, \delta\right] .
$$


Define

$$
F_{z}\left(\tau, \mathcal{C}_{\tau_{0}, \delta}\right)=\frac{1}{2} \int_{a_{\tau}}^{b_{\tau}} g\left(\dot{\eta}_{z}(\tau), \dot{\eta}_{z}(\tau)\right) d s,\left[a_{\tau}, b_{\tau}\right] \in C_{\tau_{0}, \delta}
$$

and

$$
G_{z}\left(\tau, \mathcal{C}_{\tau_{0}, \delta}\right)=\frac{b_{\tau}-a_{\tau}}{2} \int_{a_{\tau}}^{b_{\tau}} g\left(\dot{\eta}_{z}(\tau), \dot{\eta}_{z}(\tau)\right) d s,\left[a_{\tau}, b_{\tau}\right] \in C_{\tau_{0}, \delta}
$$

Then for any $\left.\tau \in] \tau_{0}, \delta\right]$ we have:

$$
F_{z}\left(\tau, C_{\tau_{0}, \delta}\right) \leq F_{z}\left(\tau_{0}, C_{\tau_{0}, \delta}\right)+L_{1}\left(\tau-\tau_{0}\right) \sqrt{2 M_{0} L_{0}}
$$

and

$$
G_{z}\left(\tau, C_{\tau_{0}, \delta}\right) \leq G_{z}\left(\tau_{0}, C_{\tau_{0}, \delta}\right)+L_{1}\left(\tau-\tau_{0}\right) \sqrt{2 M_{0} L_{0}}
$$

where $L_{0}, L_{1}$ are defined by (6.13) and (6.15) respectively.

Proof. We shall proof the statement for the map $G$, since the proof for the map $F$ is analogous and simpler.

Take $\tau>\tau_{0}$, and set $y_{\tau}=\eta_{z}(\tau)$ and $y_{\tau_{0}}=\eta_{z}\left(\tau_{0}\right)$. Since $b_{\tau_{0}}-a_{\tau_{0}} \leq 1$, and $\left[a_{\tau}, b_{\tau}\right] \subset\left[a_{\tau_{0}, \tau_{0}}\right]$, using 2.10 we have, for some $\left.\theta \in\right] \tau_{0}, \tau[)$ :

$$
\begin{gathered}
\frac{b_{\tau}-a_{\tau}}{2} \int_{a_{\tau}}^{b_{\tau}} g\left(\dot{y}_{\tau}, \dot{y}_{\tau}\right) \mathrm{d} s \leq \frac{b_{\tau_{0}}-a_{\tau_{0}}}{2} \int_{a_{\tau}}^{b_{\tau}} g\left(\dot{y}_{\tau}, \dot{y}_{\tau}\right) \mathrm{d} s=\left(b_{\tau_{0}}-a_{\tau_{0}}\right) f_{a_{\tau}, b_{\tau}}\left(\eta_{z}(\tau)\right) \\
=\left(b_{\tau_{0}}-a_{\tau_{0}}\right)\left[\left(\tau-\tau_{0}\right) \mathrm{d} f_{a_{\tau}, b_{\tau}}\left(\eta_{z}(\theta)\right)\left[\frac{\mathrm{d} \eta_{z}(\theta)}{\mathrm{d} \tau}\right]+f_{a_{\tau}, b_{\tau}}\left(\eta_{z}\left(\tau_{0}\right)\right)\right] \\
\leq\left(b_{\tau_{0}}-a_{\tau_{0}}\right) f_{a_{\tau_{0}}, b_{\tau_{0}}}\left(\eta_{z}\left(\tau_{0}\right)\right)+\left(\tau-\tau_{0}\right)\left|\mathrm{d} f_{a_{\tau}, b_{\tau}}\left(\eta_{z}(\theta)\right)\left[\frac{\mathrm{d} \eta_{z}(\theta)}{\mathrm{d} \tau}\right]\right| \\
=\frac{b_{\tau_{0}}-a_{\tau_{0}}}{2} \int_{a_{\tau_{0}}}^{b_{\tau_{0}}} g\left(\dot{y}_{\tau_{0}}, \dot{y}_{\tau_{0}}\right) \mathrm{d} s+\left(\tau-\tau_{0}\right)\left|\mathrm{d} f_{a_{\tau}, b_{\tau}}\left(\eta_{z}(\theta)\right)\left[\frac{\mathrm{d} \eta_{z}(\theta)}{\mathrm{d} \tau}\right]\right| .
\end{gathered}
$$

Note that, by 9.18 and Hölder's inequality:

$$
\begin{aligned}
\left|\mathrm{d} f_{a_{\tau}, b_{\tau}}\left(\eta_{z}(\theta)\right)\left[\frac{\mathrm{d} \eta_{z}(\theta)}{\mathrm{d} \tau}\right]\right| \leq \int_{a_{\tau}}^{b_{\tau}}\left|g\left(\frac{\mathrm{d} \eta_{z}(\theta)}{\mathrm{d} s}, \frac{\mathrm{D}}{\mathrm{d} s} W\right)\right| \mathrm{d} s \\
\quad \leq\left(\int_{a_{\tau}}^{b_{\tau}} g\left(\frac{\mathrm{d} \eta_{z}(\theta)}{\mathrm{d} s}, \frac{\mathrm{d} \eta_{z}(\theta)}{\mathrm{d} s}\right) \mathrm{d} s\right)^{\frac{1}{2}}\left(\int_{a_{\tau}}^{b_{\tau}} g\left(\frac{\mathrm{D}}{\mathrm{d} s} W, \frac{\mathrm{D}}{\mathrm{d} s} W\right) \mathrm{d} s\right)^{\frac{1}{2}} .
\end{aligned}
$$

Now

$$
\left[a_{\tau}, b_{\tau}\right] \subset\left[a_{\theta}, b_{\theta}\right] \subset\left[a_{\tau_{0}}, b_{\tau_{0}}\right]
$$

therefore by (9.19) we have

$$
\begin{aligned}
\left(\int_{a_{\tau}}^{b_{\tau}} g\left(\frac{\mathrm{d} \eta_{z}(\theta)}{\mathrm{d} s}, \frac{\mathrm{d} \eta_{z}(\theta)}{\mathrm{d} s}\right) \mathrm{d} s\right)^{\frac{1}{2}} & \\
& \leq\left(\int_{a_{\theta}}^{b_{\theta}} g\left(\frac{\mathrm{d} \eta_{z}(\theta)}{\mathrm{d} s}, \frac{\mathrm{d} \eta_{z}(\theta)}{\mathrm{d} s}\right) \mathrm{d} s\right)^{\frac{1}{2}}<\sqrt{2 M_{0}}
\end{aligned}
$$


while by (6.13), (6.15) and 9.20),

$$
\begin{aligned}
\left(\int_{a_{\tau}}^{b_{\tau}} g\left(\frac{\mathrm{D}}{\mathrm{d} s} W, \frac{\mathrm{D}}{\mathrm{d} s} W\right) \mathrm{d} s\right)^{\frac{1}{2}} \leq\left(\int_{a_{\tau}}^{b_{\tau}} L_{0}\left\|\frac{\mathrm{D}}{\mathrm{d} s} W\right\|_{E}^{2}\right)^{\frac{1}{2}} & \leq L_{1} \sqrt{L_{0}}\|W\|_{a_{\tau}, b_{\tau}} \leq L_{1} \sqrt{L_{0}} .
\end{aligned}
$$

Then

$$
\left|\mathrm{d} f_{a_{\tau}, b_{\tau}}\left(\eta_{z}(\theta)\right)\left[\frac{\mathrm{d} \eta_{z}(\theta)}{\mathrm{d} \tau}\right]\right| \leq L_{1} \sqrt{2 M_{0} L_{0}}
$$

from which we finally deduce $(9.24)$.

Lemma 9.6. Fix $x, z \in H^{1}\left([0,1], \mathbb{R}^{N}\right), \hat{\rho}>0$ and $[a, b] \subset[0,1]$ such that

$$
\|z-x\|_{a, b}<\hat{\rho} .
$$

Then there exists $\rho=\rho(x, z, a, b)$ such that, for any $w \in H^{1}\left([0,1], \mathbb{R}^{N}\right)$ such that $\| z-$ $w \|_{a, b}<\rho$, and for any $[\tilde{a}, \tilde{b}] \subset[a, b]$ it is $\|w-x\|_{\tilde{a}, \tilde{b}}<\hat{\rho}$.

Proof.

$$
\|w-x\|_{\tilde{a}, \tilde{b}} \leq\|w-x\|_{a, b} \leq\|w-z\|_{a, b}+\|z-x\|_{a, b}<\rho+\|z-x\|_{a, b},
$$

from which we deduce the thesis.

Proof of Proposition 9.1. The proof is divided into two parts: first part requires the construction of the vector field (with the help of Propositions 6.1 and 6.4) generating the flow that we shall study in the second part of the proof.

\section{Part A: Construction of the vector field.} it is:

Fix $\left.\varepsilon \in] 0, \varepsilon_{1}\right], \varepsilon_{1}<\frac{c_{1}}{4}$. Let $(\mathcal{D}, h) \in \mathcal{H}, x=h(1, \xi)$ with $\xi \in \mathcal{D}$. By assumption 9.2

$$
\left\|y-x_{\mid\left[a_{x}, b_{x}\right]}\right\|_{a_{x}, b_{x}} \geq r \text { for any } y \in \mathcal{Z}_{a_{x}, b_{x}},
$$

for any $\left[a_{x}, b_{x}\right] \in \mathcal{I}_{x}$ such that,

$$
\left(b_{x}-a_{x}\right) \int_{a_{x}}^{b_{x}} \frac{1}{2} g(\dot{x}, \dot{x}) d s \geq c_{1}-\varepsilon,
$$

Note that by the definition of $\mathfrak{M}$ we also have

$$
\int_{a_{x}}^{b_{x}} g(\dot{x}, \dot{x}) d s<M_{0}, \text { for any }\left[a_{x}, b_{x}\right] \in \mathcal{I}_{x} .
$$

For any $x \in h(1, \mathcal{D})$ we shall consider the following class of intervals:

$$
\left.\mathcal{I}_{x, r, \varepsilon}=\left\{[a, b] \in \mathcal{I}_{x}:[a, b] \text { satisfies } 9.25 \text { and } 9.26\right]\right\} \text {. }
$$

Now for any $x \in h(1, \mathcal{D})$ we choose a vector field $V_{x}$ as follows. Consider the quantities $\mu(r), \theta(r)$ and $\kappa(r)$ given in Proposition 6.1. If $x=h(1, \xi)$ on any $\left[a_{x}, b_{x}\right] \in \mathcal{I}_{x, r, \varepsilon}$ we choose as $V_{x}$ the vector field defined in $\left[a_{x}-\Delta\left(x,\left[a_{x}, b_{x}\right]\right), b_{x}+\Delta\left(x,\left[a_{x}, b_{x}\right]\right)\right]$ and given by Proposition 6.4 (satisfying (1), (2) and (3) of Proposition 6.1 with $[a, b]$ replaced by $\left.\left[a_{x}, b_{x}\right]\right)$ and such that

$$
\left\|V_{x}\right\|_{a_{x}, b_{x}}=\frac{1}{2} .
$$

If $\left[a_{x}, b_{x}\right] \in \mathcal{I}_{x} \backslash \mathcal{I}_{x, r, \varepsilon}$ we choose $V_{x}=0$ on $\left[a_{x}, b_{x}\right]$. Clearly we can choose $V_{\mathcal{R} x}$ so that

$$
V_{\mathcal{R} x}(s)=V_{x}(1-s)=\mathcal{R} V_{x}(s) \text { for any } s \in[0,1] .
$$

Indeed we have $a_{\mathcal{R} x}=1-b_{x}$ and $b_{\mathcal{R} x}=1-a_{x}$. 
Consider also $\rho(r)$ be as in Proposition 6.4. Choose $K_{*}=E(r), \rho_{*}=\frac{c_{1} E(r)}{32 M_{0} \widehat{K}}$ and

$$
\rho_{0}(r)=\min \left\{\rho(r), \rho_{*}\right\}
$$

Now by Lemma 2.1 the number of intervals $\left[a_{x}, b_{x}\right] \in \mathcal{I}_{x, r, \varepsilon}$ is finite and bounded by a constant independent of $x$. Then for any $x \in h(1, \mathcal{D})$ and $\left[a_{x}, b_{x}\right] \in \mathcal{I}_{x, r, \varepsilon}$, we can choose $\Delta(x)$ in Proposition 6.4, independently by $\left[a_{x}, b_{x}\right] \in \mathcal{I}_{x, r, \varepsilon}$, intervals $\left[\alpha_{x}, \beta_{x}\right],\left[\alpha_{x}^{\prime}, \beta_{x}^{\prime}\right]$ corresponding to $\left[a_{x}, b_{x}\right] \in \mathcal{I}_{x, r, \varepsilon}$ verifying

$$
\alpha_{\mathcal{R} x}=1-\beta_{x}, \alpha_{\mathcal{R} x}^{\prime}=1-\beta_{x}^{\prime}, \beta_{\mathcal{R} x}=1-\alpha_{x}, \beta_{\mathcal{R} x}^{\prime}=1-\alpha_{x}^{\prime},
$$

and

$$
\left[a_{x}-\Delta(x), b_{x}+\Delta(x)\right] \cap[0,1] \supset\left[\alpha_{x}, \beta_{x}\right] \supset\left[\alpha_{x}^{\prime}, \beta_{x}^{\prime}\right] \supset\left[a_{x}, b_{x}\right]
$$

and a number

such that the following properties are satisfied:

$$
\left.\rho(x) \in] 0, \frac{1}{2} \rho_{0}(r)\right]
$$

- if $\left[a_{x}^{i}, b_{x}^{i}\right]$ are the intervals in $\mathcal{I}_{x, r, \varepsilon}$, and $\left[\alpha_{x}^{i}, \beta_{x}^{i}\right]$ are the corresponding intervals above including $\left[a_{x}^{i}, b_{x}^{i}\right]$, it is

$$
i \neq j \Longrightarrow\left[\alpha_{x}^{i}, \beta_{x}^{i}\right] \cap\left[\alpha_{x}^{j}, \beta_{x}^{j}\right]=\emptyset,
$$

- $\alpha_{x}$ and $\beta_{x}$ satisfy (9.5) and (9.6) of Lemma 9.2 with $K_{*}=E(r)$,

- if $a_{x}>0$, then $\alpha_{x}<\alpha_{x}^{\prime}<a_{x}$, while if $a_{x}=0$ then $\alpha_{x}=\alpha_{x}^{\prime}=0$,

- if $b_{x}<1$, then $\beta_{x}>\beta_{x}^{\prime}>b_{x}$, while if $b_{x}=1$ then $\beta_{x}=\beta_{x}^{\prime}=0$,

- $\sup \left\{\phi\left(x(s): s \in\left[\alpha_{x}, \beta_{x}\right]\right\} \leq \frac{\delta_{0}}{4}\left(\right.\right.$ recall that $\sup \left\{\phi\left(x(s): s \in\left[a_{x}, b_{x}\right]\right\}=0\right)$,

- $\inf \left\{\phi\left(x(s): s \in\left[\alpha_{x}, \beta_{x}\right] \backslash\left[a_{x}, b_{x}\right]\right\} \geq-\frac{\kappa(r)}{2}\right.$,

- $\phi(x(s))>0$ for any $s \in\left[\alpha_{x}, \beta_{x}\right] \backslash\left[\alpha_{x}^{\prime}, \beta_{x}^{\prime}\right]^{2}$,

and for any $z \in h(1, \mathcal{D})$ also the following properties are satisfied:

- $\|x-z\|_{0,1}<\rho(x)$ implies $\int_{a_{z}^{\prime}}^{b_{z}^{\prime}} \frac{1}{2} g(\dot{z}, \dot{z}) d s<\frac{c_{1}}{2}<c_{1}-\varepsilon$, for any $\left[a_{z}^{\prime}, b_{z}^{\prime}\right] \subset$ $\left.\left[a_{z}, b_{z}\right] \in \mathcal{I}_{z},\left[a_{z}^{\prime}, b_{z}^{\prime}\right] \subset\left[\alpha_{x}, \beta_{x}\right] \backslash\right] a_{x}, b_{x}[$,

- if $\|x-z\|_{0,1}<\rho(x)$ and $x=h(1, \xi)$ has intervals in $\mathcal{I}_{x, r, \varepsilon}$, then $z$ is not constant,

- $\|x-z\|_{L^{\infty}\left([0,1], \mathbb{R}^{N}\right)}<\rho(x)$ implies $\sup \left\{\phi\left(z(s): s \in\left[\alpha_{x}, \beta_{x}\right]\right\} \leq \frac{\delta_{0}}{2}\right.$,

- $\|x-z\|_{L^{\infty}\left([0,1], \mathbb{R}^{N}\right)}<\rho(x)$ implies inf $\left\{\phi\left(x(s): s \in\left[\alpha_{x}, \beta_{x}\right] \backslash\left[a_{x}, b_{x}\right]\right\} \geq-\kappa(r)\right.$,

- $\|x-z\|_{L^{\infty}\left([0,1], \mathbb{R}^{N}\right)}<\rho(x)$ implies $\phi(z(s))>0$ for any $s \in\left[\alpha_{x}, \beta_{x}\right] \backslash\left[\alpha_{x}^{\prime}, \beta_{x}^{\prime}\right]$, whenever $\left[\alpha_{x}, \beta_{x}\right] \supset\left[a_{x}, b_{x}\right]$ with $\left[a_{x}, b_{x}\right] \in \mathcal{I}_{x, r, \varepsilon}$.

Observe that, by the last property above, it is

$$
\begin{array}{r}
\|x-z\|_{L^{\infty}\left([0,1], \mathbb{R}^{N}\right)}<\rho(x),\left[a_{z}, b_{z}\right] \in \mathcal{I}_{z},\left[a_{z}, b_{z}\right] \cap\left[\alpha_{x}, \beta_{x}\right] \neq \emptyset, \text { and }\left[a_{x}, b_{x}\right] \in \mathcal{I}_{x, r, \varepsilon} \\
\operatorname{imply}\left[a_{z}, b_{z}\right] \subset\left[\alpha_{x}^{\prime}, \beta_{x}^{\prime}\right] .
\end{array}
$$

Define now a new vector field $\widehat{V}_{x}$ as follows. Suppose $\alpha_{x}<a_{x}<b_{x}<\beta_{x}$ Let $\varphi_{x}: \mathbb{R} \rightarrow[0,1]$ be the continuous piecewise affine function which is zero outside any $\left[\alpha_{x}, \beta_{x}\right]$ as above and it is equal to 1 in any $\left[\alpha_{x}^{\prime}, \beta_{x}^{\prime}\right]$ as above. We set

$$
\widehat{V}_{x}(s)=\varphi_{x}(s) V_{x}(s) \text { for any } s \in\left[\alpha_{x}, \beta_{x}\right] \text { as above, and } 0 \text { otherwise. }
$$

If $a_{x}=0$ we choose $\varphi_{x}$ so that $\varphi_{x}(0)=1$, while if $b_{x}=1$ we choose $\varphi_{x}$ so that $\varphi_{x}(1)=1$.

A straightforward computation shows that there exists $C_{x}>0$ such that

$$
\left\|\widehat{V}_{x}(s)\right\|_{0,1} \leq C_{x}
$$

and thanks to (1.9), 9.28) and the definition of $\varphi_{x}$ we see that

$$
\left\|\widehat{V}_{x}(s)\right\|_{L^{\infty}\left([0,1], \mathbb{R}^{N}\right)} \leq \frac{1}{2} .
$$


For any $x \in h(1, \mathcal{D})$ consider the open subset of $h(1, \mathcal{D})$ given by

$$
\mathcal{U}_{x}=\left\{z \in h(1, \mathcal{D}):\|z-x\|_{0,1}<\rho(x)\right\} .
$$

Consider the open covering $\left\{\mathcal{U}_{x}, \mathcal{U}_{\mathcal{R} x}\right\}_{x \in h(1, \mathcal{D})}$ of $h(1, \mathcal{D})$. Since $h(1, \mathcal{D})$ is compact there exists a finite covering $\left\{\mathcal{U}_{x_{i}}, \mathcal{U}_{\mathcal{R} x_{i}}\right\}_{i=1, \ldots, k}$ of $h(1, \mathcal{D})$.

Define, for any $z \in h(1, \mathcal{D})$,

$$
\varrho_{x_{i}}(z)=\operatorname{dist}_{0,1}\left(z, h(1, \mathcal{D}) \backslash \mathcal{U}_{x_{i}}\right) .
$$

where dist ${ }_{0,1}$ is the distance induced by the norm $\|\cdot\|_{0,1}$. Since $\mathcal{R} h(1, \mathcal{D})=\mathcal{D}, \mathcal{R} \mathcal{U}_{x_{i}}=$ $\mathcal{U}_{\mathcal{R} x_{i}}$ and $\mathcal{R} \circ \mathcal{R}$ is the identity map, it is

$$
\varrho_{x_{i}}(\mathcal{R} z)=\varrho_{\mathcal{R} x_{i}}(z) .
$$

Finally set

Note that by 9.35

$$
\beta_{x_{i}}(z)=\frac{\varrho_{x_{i}}(z)}{\sum_{j=1, \ldots, k}\left(\rho_{x_{j}}(z)+\varrho_{\mathcal{R} x_{j}}(z)\right)} .
$$

$$
\beta_{x_{i}}(\mathcal{R} z)=\beta_{\mathcal{R} x_{i}}(z)
$$

and that $\left\{\beta_{x_{i}}, \beta_{\mathcal{R} x_{i}}\right\}_{i=1, \ldots, k}$ is a continuous partition of the unity. Indeed

$$
\sum_{j=1, \ldots, k}\left(\beta_{x_{j}}(z)+\beta_{\mathcal{R} x_{j}}(z)\right)=1 .
$$

The vector field that we want to construct is defined as

$$
W^{\lambda}(z, \eta)=\left(\sum_{j=1, \ldots, k}\left(\beta_{x_{j}}(z) \widehat{V}_{x_{j}}+\beta_{\mathcal{R} x_{j}}(z) \widehat{V}_{\mathcal{R} x_{j}}\right)+\lambda \nabla \phi(\eta)\right) \chi(\phi(\eta)),
$$

where $\lambda \in] 0,1], z \in h(1, \mathcal{D}), \eta \in \mathfrak{M}_{0}$, and

$$
\chi \in C^{2}(\mathbb{R},[0,1]) \text { satisfies: } \chi(t)=1 \forall t \leq \frac{\delta_{0}}{2}, \chi(t)=0 \forall t \geq \frac{3 \delta_{0}}{4} .
$$

Note that by the definition of $\widehat{V}_{x}$, the choice of $\rho(x)$, the definition of $\chi$, (1.9), (1.12), (9.33) and (6.13) we have

$$
\left.\left.\left\|W^{\lambda}(z, \eta)\right\|_{L^{\infty}\left([0,1], \mathbb{R}^{N}\right)} \leq \frac{1}{2}+\lambda \frac{K_{0}}{\sqrt{\ell_{0}}} \leq 1, \forall \lambda \in\right] 0, \frac{\sqrt{\ell_{0}}}{2 K_{0}}\right],
$$

and there exists constants $C_{1}, C_{2} \in \mathbb{R}^{+}$such that

$$
\left\|W^{\lambda}(z, \eta)\right\|_{0,1} \leq C_{1}+\lambda C_{2}\|\eta\|_{0,1} .
$$

Moreover, by (9.29), the $\mathcal{R}$-equivariant choice of $\alpha_{x}, \alpha_{x}^{\prime}, \beta_{x}^{\prime}, \beta_{x}$ and the definition of the map $\varphi_{x}$, we have $\widehat{V}_{\mathcal{R} x}=\mathcal{R} \widehat{V}_{x}$, so by $(9.36)$

$$
\mathcal{R} W^{\lambda}(z, \eta)=W^{\lambda}(\mathcal{R} z, \mathcal{R} \eta) .
$$

The existence of the homotopy $H_{\varepsilon}$ will be proved studying the flow given by the solution of the initial value problem

$$
\left\{\begin{array}{l}
\frac{\mathrm{d}}{\mathrm{d} \tau} \eta=W^{\lambda}(z, \eta) \\
\eta(0)=z \in h(1, \mathcal{D}) .
\end{array}\right.
$$

Note that by (9.41) the solution $\eta(\tau, z)$ of $(9.43)$ is defined for all $\tau \in \mathbb{R}^{+}$, taking values on $H^{1}\left([0,1], \mathbb{R}^{N}\right)$ for any $z \in h(1, \mathcal{D})$.

Part B: Study of the flow $\eta(\tau, z)$. 
Step 1. The flow $\eta$ is $\mathcal{R}$-equivariant, namely

$$
\eta(\tau, \mathcal{R} z)=\mathcal{R} \eta(\tau, z)
$$

Proof. It is sufficient to observe that $\mathcal{R} \eta(\tau, z)$ solves the Cauchy problem

$$
\left\{\begin{array}{l}
\frac{\mathrm{d}}{\mathrm{d} \tau} \eta=W^{\lambda}(\mathcal{R} z, \eta) \\
\eta(0)=\mathcal{R} z
\end{array}\right.
$$

But this is a simple consequence of (9.42) and the $\mathcal{R}$-equivariance of $h$.

Step 2. If $z$ is a constant curve, then $\eta(\tau, z)$ is a constant curve for all $\tau \geq 0$.

Proof. If $z$ is a constant curve, by the choice of $\rho(x)$ and 9.31 we have:

$$
z \in \mathcal{U}_{x_{i}} \Longrightarrow \widehat{V}_{x_{i}} \equiv 0 \text {, and } \widehat{V}_{\mathcal{R} x_{i}} \equiv 0 \text {. }
$$

Then $\eta$ solves

$$
\left\{\begin{array}{l}
\frac{\mathrm{d}}{\mathrm{d} \tau} \eta=\lambda \nabla \phi(\eta) \chi(\phi(\eta)) \\
\eta(0)=z .
\end{array}\right.
$$

Denote by $\eta_{\tau}$ the integral flow in $\mathbb{R}^{N}$ of the above Cauchy problem, where the constant curve $z$ can be identified with a vector in $\mathbb{R}^{N}$. By the local uniqueness property we deduce that, for any $\tau \geq 0, \eta(\tau, z)$ is the constant curve $\eta_{\tau}$.

Step 3. Fix $\left.\lambda \in] 0, \frac{\sqrt{\ell_{0}}}{2 K_{0}}\right]$. Then for any $z \in \mathcal{U}_{x_{i}}$ if $\tau_{*} \in \mathbb{R}^{+}$is the first instant such that $\left\|\eta(\tau, z)-x_{i}\right\|_{L^{\infty}\left([0,1], \mathbb{R}^{N}\right)}=\rho(r)$ (where $\rho(r)$ is given by Proposition 6.4), then $\tau_{*} \geq \frac{\rho(r)}{2}$.

Proof. Since $\rho\left(x_{i}\right) \leq \frac{\rho(r)}{2}$ if $z \in \mathcal{U}_{x_{i}}$, by (9.40) and 9.43) we have

$$
\begin{aligned}
\frac{\rho(r)}{2} \leq & \left\|\eta\left(\tau_{*}, z\right)-x_{i}\right\|_{L^{\infty}\left([0,1], \mathbb{R}^{N}\right)}-\left\|\eta(0, z)-x_{i}\right\|_{L^{\infty}\left([0,1], \mathbb{R}^{N}\right)} \leq \\
& \left\|\eta\left(\tau_{*}, z\right)-\eta(0, z)\right\|_{L^{\infty}\left([0,1], \mathbb{R}^{N}\right)} \leq \int_{0}^{\tau_{*}}\left\|\frac{\mathrm{d}}{\mathrm{d} \sigma} \eta(\sigma, z)\right\|_{L^{\infty}\left([0,1], \mathbb{R}^{N}\right)} \mathrm{d} \sigma \leq \tau_{*} .
\end{aligned}
$$

Step 4. Fix $\left.\lambda \in] 0, \frac{\sqrt{\ell_{0}}}{2 K_{0}}\right]$. Then, for any $\tau \in\left[0, \frac{\rho(r)}{2}\right], \eta(\tau, z)$ satisfies (11) and (2) $)$ of Definition 8.1.

Proof. To prove (11) of Definition 8.1. first of all observe that the curve $[0,1] \ni s \mapsto$ $\eta(\tau, z)(s) \in \mathbb{R}^{N}$ is of class $H^{1}$, and the map $\tau \mapsto \eta(\tau, z)(\cdot)$ is of class $C^{1}$ and taking values in $H^{1}\left([0,1], \mathbb{R}^{N}\right)$.

Therefore, for any $s \in[0,1]$ we have:

$$
\begin{gathered}
\frac{\partial}{\partial \tau} \phi(\eta(\tau, z)(s))=g\left(\nabla \phi(\eta(\tau, z)(s)), \frac{\partial}{\partial \tau} \eta(\tau, z)(s)\right)= \\
g\left(\nabla \phi(\eta(\tau, z)(s)), W^{\lambda}(\eta(\tau, z)(s))\right)= \\
g\left(\nabla \phi\left(\eta(\tau, z)(s), \sum_{j=1}^{k} \beta_{x_{j}}(z) \varphi_{x_{j}}(s) V_{x_{j}}(s)\right) \chi(\phi(\eta(\tau, z)(s))+\right. \\
g\left(\nabla \phi\left(\eta(\tau, z)(s), \sum_{j=1}^{k} \beta_{\mathcal{R} x_{j}}(z) \varphi_{\mathcal{R} x_{j}}(s) V_{\mathcal{R} x_{j}}(s)\right) \chi(\phi(\eta(\tau, z)(s)))+\right. \\
\lambda g(\nabla \phi(\eta(\tau, z)(s)), \nabla \phi(\eta(\tau, z)(s))) \chi(\phi(\eta(\tau, z)(s))) .
\end{gathered}
$$


Now $\phi=0$ implies $g(\nabla \phi, \nabla \phi)>0$ and $\chi(\phi)=1$. Therefore, since $\lambda>0$, we have

$$
\begin{aligned}
\phi(\eta(\tau, z)(s))=0 \Longrightarrow \frac{\partial}{\partial \tau} \phi(\eta(\tau, z)(s))> & \\
& \sum_{j=1}^{k} \beta_{x_{j}}(z) \varphi_{x_{j}}(s) g\left(\nabla \phi(\eta(\tau, z)(s)), V_{x_{j}}(s)\right)+ \\
& \sum_{j=1}^{k} \beta_{\mathcal{R} x_{j}}(z) \varphi_{\mathcal{R} x_{j}}(s) g\left(\nabla \phi(\eta(\tau, z)(s)), V_{\mathcal{R} x_{j}}(s)\right) .
\end{aligned}
$$

Then by Step 3, (1b) of Proposition (6.4) and the choice of $V_{x_{i}}$ and $V_{\mathcal{R} x_{i}}$, we obtain the proof since, for any $j=1, \ldots, k, \varphi_{x_{j}}, \varphi_{\mathcal{R} x_{j}} \geq 0$ and $\beta_{x_{j}}, \beta_{\mathcal{R} x_{j}} \geq 0$. The proof of property (2) is completely analogous.

Set

$$
\left.\left.N_{0}=\sup \left\{\left\|H^{\phi}(y)\right\|: y \in \phi^{-1}(]-\infty, \delta_{0}\right]\right)\right\},
$$

where $\left\|H^{\phi}(y)\right\|$ is the norm induced by the metric $g$ of the hessian of the map $\phi$ at the point $y$.

Remark 9.7. Let $\left[a_{\tau}, b_{\tau}\right] \in \mathcal{I}_{\eta(\tau, z)}$ and $\eta(\tau, z) \in \mathfrak{M}$. Since, by step 4, $\left[a_{\tau}, b_{\tau}\right] \subset\left[a_{z}, b_{z}\right]$, by the definition of $W^{\lambda}$ we have

$$
\left\|W^{\lambda}(z, \eta(\tau, z))\right\|_{a_{\tau}, b_{\tau}} \leq \frac{1}{2}+\lambda\|\nabla \phi(\eta(\tau, z))\|_{a_{\tau}, b_{\tau}}
$$

Now by $(1.8),(1.12),(6.13)$ and $(9.44)$ (recalling that we are assuming that $\eta(\tau, z) \in \mathfrak{M})$

so

$$
\| \nabla \phi\left(\eta(\tau, z) \|_{a_{\tau}, b_{\tau}}<\sqrt{\frac{K_{0}^{2}}{2 \ell_{0}}+\frac{M_{0} N_{0}^{2}}{\ell_{0}}},\right.
$$

$$
\left\|W^{\lambda}(z, \eta(\tau, z))\right\|_{a_{\tau}, b_{\tau}} \leq \frac{1}{2}+\lambda \sqrt{\frac{K_{0}^{2}}{2 \ell_{0}}+\frac{M_{0} N_{0}^{2}}{\ell_{0}}} .
$$

Then if we set

$$
\lambda_{1}=\min \left(\frac{\sqrt{\ell_{0}}}{2 K_{0}}, \frac{\sqrt{\ell_{0}}}{\sqrt{2 K_{0}^{2}+4 M_{0} N_{0}^{2}}}\right),
$$

we have (recall $(9.40)$ ),

$$
\left\|W^{\lambda}(z, \eta(\tau, z))\right\|_{L^{\infty}\left([0,1], \mathbb{R}^{N}\right)} \leq 1,\left\|W^{\lambda}(z, \eta(\tau, z))\right\|_{a_{\tau}, b_{\tau}} \leq 1
$$

for any $\left.\lambda \in] 0, \lambda_{1}\right]$, for any $\tau$ such that $\eta(\tau, z) \in \mathfrak{M}$.

Step 5. Fix $\left.\lambda \in] 0, \lambda_{1}\right]$ and $z \in \mathcal{U}_{x_{i}}$ for some $i=1, \ldots, k$. Suppose that $\left.\left.\tau_{*} \in\right] 0, \frac{\rho(r)}{2}\right]$ is such that:

- $\left.\| \eta\left(\tau_{*}, z\right)-x_{i}\right) \|_{a_{\tau_{*}}, b_{\tau_{*}}} \geq \rho(r)$,

- $\left.\| \eta(\tau, z)-x_{i}\right) \|_{a_{\tau}, b_{\tau}}<\rho(r)$, for any $\tau \in\left[0, \tau_{*}[\right.$,

- $\eta\left(\tau_{*}, z\right) \in \mathfrak{M}$, for any $\tau \in\left[0, \tau_{*}[\right.$,

where $\left[a_{\tau}, b_{\tau}\right] \in \mathcal{I}_{\eta(\tau, z)}$ for any $\tau \in\left[0, \tau_{*}\right]$. Then $\tau_{*} \geq \frac{1}{2} \rho(r)$.

Proof. Since $\rho\left(x_{i}\right) \leq \frac{1}{2} \rho(r)$ and (by Step 4) $\left[a_{\tau_{*}}, b_{\tau_{*}}\right] \subset\left[a_{0}, b_{0}\right]=\left[a_{z}, b_{z}\right]$, it is

$$
\begin{aligned}
\left.\frac{1}{2} \rho(r) \leq \| \eta\left(\tau_{*}, z\right)-x_{i}\right) \|_{a_{\tau_{*}}, b_{\tau_{*}}}- & \left.\| \eta(0, z)-x_{i}\right) \|_{a_{0}, b_{0}} \leq \\
& \left.\left.\| \eta\left(\tau_{*}, z\right)-x_{i}\right)\left\|_{a_{\tau_{*}}, b_{\tau_{*}}}-\right\| \eta(0, z)-x_{i}\right) \|_{a_{\tau_{*},}, b_{\tau_{*}}} .
\end{aligned}
$$


Then

$$
\left.\frac{1}{2} \rho(r) \leq \| \eta\left(\tau_{*}, z\right)-\eta(0, z)\right)\left\|_{a_{\tau_{*}}, b_{\tau_{*}}} \leq \int_{0}^{\tau_{*}}\right\| \frac{\mathrm{d}}{\mathrm{d} \sigma} \eta(\sigma, z) \|_{a_{\tau_{*}}, b_{\tau_{*}}} d s .
$$

Now, by Step $4,\left[a_{\tau_{*}}, b_{\tau_{*}}\right] \subset\left[a_{\tau}, b_{\tau}\right]$ for any $\tau \in\left[0, \tau_{*}\right]$. Then by 9.46 ) and (9.45) it is $\left\|\frac{\mathrm{d}}{\mathrm{d} \sigma} \eta(\sigma, z)\right\|_{a_{\tau_{*}}, b_{\tau_{*}}} \leq 1$ for any $\sigma \in\left[0, \tau_{*}\right]$, from which we deduce the thesis.

Define

$$
M_{h, \mathcal{D}}=\sup \left\{\frac{1}{2} \int_{a}^{b} g(\dot{z}, \dot{z}) d s:[a, b] \in \mathcal{I}_{z}, z \in h(1, \mathcal{D})\right\}
$$

(and note that $M_{h, \mathcal{D}}<M_{0}$ since $h(1, \mathcal{D}) \subset \mathfrak{M}$ and it is compact).

Step 6. Fix

$$
\tau_{1}(r)=\min \left(\frac{\rho(r)}{2}, \frac{E(r)}{8 L_{1} \sqrt{2 M_{0} L_{0}}}, \frac{M_{0}-\frac{c_{1}}{2}}{L_{1} \sqrt{2 M_{0} L_{0}}}, 1\right) .
$$

Then for any $\left.\lambda \in] 0, \min \left(\lambda_{1}, \frac{M_{0}-M_{h, \mathcal{D}}}{2 N_{0} M_{0}}\right)\right]$ and for any $\tau \in\left[0, \tau_{1}(r)\right]$ we have $\eta(\tau, z) \in \mathfrak{M}$.

Proof. Since $W^{\lambda}(z, \eta(\tau, z))(s)=0$ whenever $\phi(\eta(\tau, z)) \geq \frac{3 \delta_{0}}{4}$ (cf. 9.38) and 9.39), by step $4 \eta(\tau, z) \in \mathfrak{M}_{0}$ for any $\tau \in\left[0, \frac{\rho(r)}{2}\right]$. We have therefore to prove that, for any $\tau \in\left[0, \tau_{1}(r)\right]:$

$$
\int_{a_{\tau}}^{b_{\tau}} \frac{1}{2} g\left(\dot{y}_{\tau}, \dot{y}_{\tau}\right) d s<M_{0}, y_{\tau}=\eta(\tau, z), \forall\left[a_{\tau}, b_{\tau}\right] \in \mathcal{I}_{\eta(\tau, z)} .
$$

First of all consider a NIIC $\mathcal{C}_{0, \tau_{1}(r)}=\left\{\left[a_{\tau}^{\prime}, b_{\tau}^{\prime}\right]: \tau \in\left[0, \tau_{1}(r)\right]\right\}$ such that

$$
\left[a_{\tau}^{\prime}, b_{\tau}^{\prime}\right] \subset\left[a_{\tau}, b_{\tau}\right] \in \mathcal{I}_{\eta(\tau, z)}
$$

and there exists $\tau_{0} \in\left[0, \tau_{1}(r)\left[\right.\right.$ such that $\left[a_{\tau_{0}}^{\prime}, b_{\tau_{0}}^{\prime}\right] \in \mathcal{C}_{0, \tau_{1}(r)}$ satisfies

$$
\begin{aligned}
& \exists i \in\{1, \ldots, k\}:\left[a_{\tau_{0}}^{\prime}, b_{\tau_{0}}^{\prime}\right] \subset\left[a_{z}, b_{z}\right] \in \mathcal{I}_{z}, z \in \mathcal{U}_{x_{i}}, \\
& \text { and } \left.\left[a_{\tau_{0}}^{\prime}, b_{\tau_{0}}^{\prime}\right] \subset\left[\alpha_{x_{i}}^{\prime}, \beta_{x_{i}}^{\prime}\right] \backslash\right] a_{x_{i}}, b_{x_{i}}\left[, \text { for some }\left[a_{x_{i}}, b_{x_{i}}\right] \in \mathcal{I}_{x_{i}} .\right.
\end{aligned}
$$

Note that, by the choice of $\rho\left(x_{i}\right)$ it is $\int_{a_{\tau_{0}}^{\prime}}^{b_{\tau_{0}}^{\prime}} \frac{1}{2} g(\dot{z}, \dot{z}) d s<\frac{c_{1}}{2}$, therefore since $\tau_{1}(r)$ satisfies

$$
\frac{c_{1}}{2}+L_{1} \tau_{1}(r) \sqrt{2 M_{0} L_{0}} \leq M_{0}
$$

by Lemma 9.5 we have:

(9.51) if $\left[a_{\tau_{0}}^{\prime}, b_{\tau_{0}}^{\prime}\right] \in \mathcal{C}_{0, \tau_{1}(r)}$ satisfies 9.50 for some $i \in\{1, \ldots, k\}$ then

$$
\int_{a_{\tau}^{\prime}}^{b_{\tau}^{\prime}} \frac{1}{2} g\left(\dot{y}_{\tau}, \dot{y}_{\tau}\right) d s<M_{0}, y_{\tau}=\eta(\tau, z), \forall\left[a_{\tau}^{\prime}, b_{\tau}^{\prime}\right] \in \mathcal{C}_{0, \tau_{1}(r)} .
$$

Now choose a NIIC $\widehat{\mathcal{C}}_{0, \tau_{1}(r)}$ consisting of intervals $\left[a_{\tau} . b_{\tau}\right]$ such that

$$
\left[a_{\tau} . b_{\tau}\right] \in \mathcal{I}_{\eta(\tau, z)} \text { for any } \tau \in\left[0, \tau_{1}(r)\right],
$$

and consider the following partition of the set of indexes $\{1, \ldots, k\}$ (recalling that, by (9.30), any $\left[a_{\tau}, b_{\tau}\right]$ intersects at most one interval in $\left.\mathcal{I}_{x_{i}, r, \varepsilon}\right)$ :

$$
\begin{gathered}
I_{1}^{\tau}=\left\{i=1, \ldots, k:\left[a_{\tau}, b_{\tau}\right] \cap\left[a_{x_{i}}, b_{x_{i}}\right] \neq \emptyset \text { for some }\left[a_{x_{i}}, b_{x_{i}}\right] \in \mathcal{I}_{x_{i}, r, \varepsilon}\right\}, \\
I_{2}^{\tau}=\left\{j=1, \ldots, k:\left[a_{\tau}, b_{\tau}\right] \subset\left[\alpha_{x_{j}}^{\prime}, \beta_{x_{j}}^{\prime}\right]\right. \\
\text { and } \left.\left[a_{\tau}, b_{\tau}\right] \cap\left[a_{x_{j}}, b_{x_{j}}\right]=\emptyset \text { for some }\left[a_{x_{j}}, b_{x_{j}}\right] \in \mathcal{I}_{x_{j}, r, \varepsilon}\right\}, \\
I_{3}^{\tau}=\left\{m=1, \ldots, k:\left[a_{\tau}, b_{\tau}\right] \cap\left[\alpha_{x_{m}}, \beta_{x_{m}}\right]=\emptyset\right. \\
\text { for any } \left.\left[\alpha_{x_{m}}, \beta_{x_{m}}\right] \text { having the corresponding }\left[a_{x_{m}}, b_{x_{m}}\right] \in \mathcal{I}_{x_{m}, r, \varepsilon}\right\}, \\
J_{1}^{\tau}=\left\{i=1, \ldots, k:\left[a_{\tau}, b_{\tau}\right] \cap\left[a_{\mathcal{R} x_{i}}, b_{\mathcal{R} x_{i}}\right] \neq \emptyset \text { for some }\left[a_{\mathcal{R} x_{i}}, b_{\mathcal{R} x_{i}}\right] \in \mathcal{I}_{\mathcal{R} x_{i}, r, \varepsilon}\right\},
\end{gathered}
$$




$$
\begin{aligned}
& J_{2}^{\tau}=\left\{j=1, \ldots, k:\left[a_{\tau}, b_{\tau}\right] \subset\left[\alpha_{\mathcal{R} x_{j}}^{\prime}, \beta_{\mathcal{R} x_{j}}^{\prime}\right]\right. \\
& \left.\quad \text { and }\left[a_{\tau}, b_{\tau}\right] \cap\left[a_{\mathcal{R} x_{j}}, b_{\mathcal{R} x_{j}}\right]=\emptyset \text { for some }\left[a_{\mathcal{R} x_{j}}, b_{\mathcal{R} x_{j}}\right] \in \mathcal{I}_{\mathcal{R} x_{j}, r, \varepsilon}\right\}, \\
& J_{3}^{\tau}=\left\{m=1, \ldots, k:\left[a_{\tau}, b_{\tau}\right] \cap\left[\alpha_{\mathcal{R} x_{m}}, \beta_{\mathcal{R} x_{m}}\right]=\emptyset\right. \\
& \left.\quad \text { for any }\left[\alpha_{\mathcal{R} x_{m}}, \beta_{\mathcal{R} x_{m}}\right] \text { having the corresponding }\left[a_{\mathcal{R} x_{m}}, b_{\mathcal{R} x_{m}}\right] \in \mathcal{I}_{\mathcal{R} x_{m}, r, \varepsilon}\right\} .
\end{aligned}
$$

Note that, by (9.51), we can just consider the case $I_{2}^{\tau} \cup J_{2}^{\tau}=\emptyset$ for any $\tau \in\left[0, \tau_{1}(r)\right]$, studying first the case:

(9.52) there exist $\tau_{*} \in\left[0, \tau_{1}(r)\right]$ and $i \in I_{1}^{\tau_{*}} \cup J_{1}^{\tau_{*}}$ such that:

$$
\begin{gathered}
{\left[a_{\tau_{*}}, b_{\tau_{*}}\right] \cap\left[a_{x_{i}}, b_{x_{i}}\right] \neq \emptyset \text { for some }\left[a_{x_{i}}, b_{x_{i}}\right] \in \mathcal{I}_{x_{i}, r, \varepsilon}} \\
\text { and } D\left(x_{i}, a_{\tau_{*}}, b_{\tau_{*}}, a_{x_{i}}, b_{x_{i}}\right)>E(r) \text { or } \\
{\left[a_{\tau_{*}}, b_{\tau_{*}}\right] \cap\left[a_{\mathcal{R} x_{i}}, b_{\mathcal{R} x_{i}}\right] \neq \emptyset \text { for some }\left[a_{\mathcal{R} x_{i}}, b_{\mathcal{R} x_{i}}\right] \in \mathcal{I}_{\mathcal{R} x_{i}, r, \varepsilon}} \\
\text { and } D\left(\mathcal{R} x_{i}, a_{\tau_{*}}, b_{\tau_{*}}, a_{\mathcal{R} x_{i}}, b_{\mathcal{R} x_{i}}\right)>E(r) .
\end{gathered}
$$

In this case by Lemma 9.2 applied with $K_{*}=E(r)$ we deduce that

$$
\int_{a_{\tau_{*}}}^{b_{\tau_{*}}} \frac{1}{2} g\left(\dot{y}_{\tau_{*}}, \dot{y}_{\tau_{*}}\right) d s<M_{0}-\frac{E(r)}{8}, y_{\tau}=\eta\left(\tau_{*}, z\right)
$$

Then by Lemma 9.5, for any $\tau \geq \tau_{*}$ we have

$$
\int_{a_{\tau}}^{b_{\tau}} \frac{1}{2} g\left(\dot{y}_{\tau}, \dot{y}_{\tau}\right) d s<M_{0}-\frac{E(r)}{8}+L_{1}\left(\tau-\tau_{*}\right) \sqrt{2 M_{0} L_{0}}
$$

provided that $M_{0}-\frac{E(r)}{8}+L_{1}\left(\tau-\tau_{*}\right) \sqrt{2 M_{0} L_{0}} \leq M_{0}$. Then, since $\tau_{1}(r)>0$ satisfies (cf. 9.48)

$$
L_{1} \tau_{1}(r) \sqrt{2 M_{0} L_{0}}-\frac{E(r)}{8} \leq 0,
$$

we have the following property:

if there exist $\tau_{*} \in\left[0, \tau_{1}(r)\right]$ and $i \in I_{1}^{\tau_{*}} \cup J_{1}^{\tau_{*}}$ such that (9.52) is satisfied, then

$$
\int_{a_{\tau}}^{b_{\tau}} \frac{1}{2} g\left(\dot{y}_{\tau}, \dot{y}_{\tau}\right) d s<M_{0} \text { for any } \tau \in\left[\tau_{*}, \tau_{1}(r)\right], \text { for any }\left[a_{\tau}, b_{\tau}\right] \in \widehat{\mathcal{C}}_{\tau_{*}, \tau_{1}(r)} .
$$

Therefore, recalling that we have just to consider the case $I_{2}^{\tau} \cup J_{2}^{\tau}=\emptyset$ for any $\tau \in$ $\left[0, \tau_{1}(r)\right]$, to conclude the proof of step 6 it will be sufficient to prove that

$$
\int_{a_{\tau}}^{b_{\tau}} \frac{1}{2} g\left(\dot{y}_{\tau}, \dot{y}_{\tau}\right) d s<M_{0} \text { for any } \tau \in\left[0, \tau_{1}(r)\right], \text { for any }\left[a_{\tau}, b_{\tau}\right] \in \widehat{\mathcal{C}}_{0, \tau_{1}(r)},
$$

under the assumptions

$$
\begin{aligned}
& \left.\forall \hat{\tau} \in] 0, \tau_{1}(r)\right], \forall \tau \in[0, \hat{\tau}], \forall i \in I_{1}^{\tau} \cup J_{1}^{\tau} \text { it is } \\
& {\left[a_{\tau_{*}}, b_{\tau_{*}}\right] \cap\left[a_{x_{i}}, b_{x_{i}}\right] \neq \emptyset \text { with }\left[a_{x_{i}}, b_{x_{i}}\right] \in \mathcal{I}_{x_{i}, r, \varepsilon}} \\
& \text { and } D\left(x_{i}, a_{\tau}, b_{\tau}, a_{x_{i}}, b_{x_{i}}\right) \leq E(r) \text { and } \\
& {\left[a_{\tau_{*}}, b_{\tau_{*}}\right] \cap\left[a_{\mathcal{R} x_{i}}, b_{\mathcal{R} x_{i}}\right] \neq \emptyset \text { for some }\left[a_{\mathcal{R} x_{i}}, b_{\mathcal{R} x_{i}}\right] \in \mathcal{I}_{\mathcal{R} x_{i}, r, \varepsilon}} \\
& \text { and } D\left(\mathcal{R} x_{i}, a_{\tau}, b_{\tau}, a_{\mathcal{R} x_{i}}, b_{\mathcal{R} x_{i}}\right) \leq E(r),
\end{aligned}
$$

and

$$
I_{2}^{\tau} \cup J_{2}^{\tau}=\emptyset \text { for any } \tau \in\left[0, \tau_{1}(r)\right] .
$$

Towards this goal, for all $z \in h(1, \mathcal{D})$, set:

$$
\Phi_{z}(\tau)=F_{h(1, z)}\left(\tau, \widehat{\mathcal{C}}_{0, \tau_{1}(r)}\right) .
$$


Since $z \in \mathfrak{M}, \Phi_{z}(0)<M_{0}$. Then by Lemma 9.6, there exists $\tilde{\tau}>0$ such that $\Phi_{z}(\tau)<M_{0}$ for any $\tau \in[0, \tilde{\tau}]$. Working on $\tau \in[0, \tilde{\tau}]$, we have, for any $l$ sufficiently small,

$$
\begin{aligned}
& \Phi_{z}(\tau+l)-\Phi_{z}(\tau)= \\
& f_{a_{\tau+l}, b_{\tau+l}}(\eta(\tau+l, z))-f_{a_{\tau}, b_{\tau}}(\eta(\tau+l, z))+f_{a_{\tau}, b_{\tau}}(\eta(\tau+l, z))-f_{a_{\tau}, b_{\tau}}(\eta(\tau, z)) \\
& \stackrel{\text { by Step } 4}{\leq} f_{a_{\tau}, b_{\tau}}(\eta(\tau+l, z))-f_{a_{\tau}, b_{\tau}}(\eta(\tau, z)) \\
& \quad=l \mathrm{~d} f_{a_{\tau}, b_{\tau}}(\eta(\tau, z))\left[\frac{\mathrm{d}}{\mathrm{d} \tau} \eta(\tau, z)\right]+o(l) \quad \text { as } l \rightarrow 0 .
\end{aligned}
$$

Then setting $y_{\tau}=\eta(\tau, z)$

$$
\begin{gathered}
\limsup _{l \rightarrow 0^{+}} \frac{\Phi_{z}(\tau+l)-\Phi_{z}(\tau)}{l} \leq \int_{a_{\tau}}^{b_{\tau}} g\left(\dot{y}_{\tau}, \frac{\mathrm{D}}{\mathrm{d} s} W^{\lambda}\left(z, y_{\tau}\right)\right) d s= \\
\sum_{i=1}^{k}\left(\beta_{x_{i}}(z) \int_{a_{\tau}}^{b_{\tau}} g\left(\dot{y}_{\tau}, \frac{\mathrm{D}}{\mathrm{d} s} \widehat{V}_{x_{i}}\right) d s+\beta_{\mathcal{R} x_{i}}(z) \int_{a_{\tau}}^{b_{\tau}} g\left(\dot{y}_{\tau}, \frac{\mathrm{D}}{\mathrm{d} s} \widehat{V}_{\mathcal{R} x_{i}}\right) d s\right)+ \\
\lambda \int_{a_{\tau}}^{b_{\tau}} H^{\phi}\left(y_{\tau}\right)\left[\dot{y}_{\tau}, \dot{y}_{\tau}\right] d s
\end{gathered}
$$

Now if $i \in I_{3}^{\tau}, \widehat{V}_{x_{i}}(s)=0$ for any $s \in\left[a_{\tau}, b_{\tau}\right]$ and if $j \in J_{3}^{\tau}, \widehat{V}_{\mathcal{R} x_{j}}(s)=0$ for any $s \in\left[a_{\tau}, b_{\tau}\right]$. Therefore, by (9.54), 9.55), (2) of Proposition 6.4 and Step 5 (recalling that $\left.\tau_{1}(r) \leq \frac{\rho(r)}{2}\right)$, we have

$$
\limsup _{l \rightarrow 0^{+}} \frac{\Phi_{z}(\tau+l)-\Phi_{z}(\tau)}{l} \leq \lambda \int_{a_{\tau}}^{b_{\tau}} H^{\phi}\left(y_{\tau}\right)\left[\dot{y}_{\tau}, \dot{y}_{\tau}\right] d s<2 \lambda N_{0} M_{0}
$$

for any $\tau \in\left[0, \tau_{1}(r)\right]$ such that $\Phi_{z}(\tau)<M_{0}$. Then

$$
\Phi_{z}(\tau)<M_{0} \Longrightarrow \Phi_{z}(\tau)<\Phi_{z}(0)+2 N_{0} M_{0} \lambda \tau \leq M_{h, \mathcal{D}}+2 N_{0} M_{0} \lambda \tau,
$$

where $M_{h, \mathcal{D}}$ is defined by (9.47). Therefore, since $\tau_{1}(r) \leq 1$ and $\lambda$ satisfies

$$
M_{h, \mathcal{D}}+2 N_{0} M_{0} \lambda \leq M_{0},
$$

the proof of Step 6 is concluded.

Step 7. Fix $z \in h(1, \mathcal{D})$ and a NIIC $\mathcal{C}_{0, \tau_{1}(r)}$ consisting of intervals $\left[a_{\tau}, b_{\tau}\right] \in \mathcal{I}_{\eta(\tau, z)}$ such that

$$
\begin{aligned}
{\left[a_{0}, b_{0}\right] \in \mathcal{I}_{z} \text { and } z \in \mathcal{U}_{x_{i}} \Longrightarrow\left[a_{0}, b_{0}\right] \cap\left[a_{x_{i}}, b_{x_{i}}\right] } & \neq \emptyset, \\
& \text { for some }\left[a_{x_{i}}, b_{x_{i}}\right] \in \mathcal{I}_{x_{i}, r, \varepsilon} .
\end{aligned}
$$

Set

$$
\Psi_{z}(\tau)=G_{z}\left(\tau, \widehat{\mathcal{C}}_{0, \tau_{1}(r)}\right),
$$

cf. (9.22). Then there exists $\left.\left.\tau_{2}(r) \in\right] 0, \tau_{1}(r)\right]$ and $\left.\left.\lambda_{2} \in\right] 0, \min \left(\lambda_{1}, \frac{M_{0}-M_{h, \mathcal{D}}}{2 N_{0} M_{0}}\right)\right]$ such that

$$
\limsup _{l \rightarrow 0^{+}} \frac{\Psi_{z}(\tau+l)-\Psi_{z}(\tau)}{l} \leq-\frac{\mu(r) c_{1}}{16 M_{0}} \text { for any } \tau \in\left[0, \tau_{2}(r)\right]
$$

or

(9.59) $\exists \tau_{*} \in\left[0, \tau_{2}(r)\right]$ such that 9.58$)$ holds for any $\tau \in\left[0, \tau_{*}[\right.$ and

$$
\Psi_{z}(\tau) \leq \Psi_{z}(0)-\min \left(\frac{c_{1} E(r)}{64 M_{0}}, \frac{c_{1}}{4}\right) \text { for any } \tau \in\left[\tau_{*}, \tau_{2}(r)\right] .
$$


Proof. Fix $l>0$. By Step 4

$$
\Psi_{z}(\tau+l)-\Psi_{z}(\tau) \leq\left(b_{\tau}-a_{\tau}\right)\left(\Phi_{z}(\tau+l)-\Phi_{z}(\tau)\right),
$$

where $\left[a_{\tau}, b_{\tau}\right] \in \widehat{\mathcal{C}}_{0, \tau_{1}(r)}$. Then setting $y_{\tau}=\eta(\tau, z)$ by (9.56) we have

$$
\begin{array}{r}
\limsup _{l \rightarrow 0^{+}} \frac{\Psi_{z}(\tau+l)-\Psi_{z}(\tau)}{l} \leq\left(b_{\tau}-a_{\tau}\right) \int_{a_{\tau}}^{b_{\tau}} g\left(\dot{y}_{\tau}, \frac{\mathrm{D}}{\mathrm{d} s} W^{\lambda}\left(z, y_{\tau}\right)\right) d s= \\
\left(b_{\tau}-a_{\tau}\right) \sum_{i=1}^{k}\left(\beta_{x_{i}}(z) \int_{a_{\tau}}^{b_{\tau}} g\left(\dot{y}_{\tau}, \frac{\mathrm{D}}{\mathrm{d} s} \widehat{V}_{x_{i}}\right) d s+\beta_{\mathcal{R} x_{i}}(z) \int_{a_{\tau}}^{b_{\tau}} g\left(\dot{y}_{\tau}, \frac{\mathrm{D}}{\mathrm{d} s} \widehat{V}_{\mathcal{R} x_{i}}\right) d s\right)+ \\
\lambda\left(b_{\tau}-a_{\tau}\right) \int_{a_{\tau}}^{b_{\tau}} H^{\phi}\left(y_{\tau}\right)\left[\dot{y}_{\tau}, \dot{y}_{\tau}\right] d s .
\end{array}
$$

Consider first the case

$$
\exists \tau_{*} \in\left[0, \tau_{2}(r)\right]: \Psi_{z}(\tau)<\frac{c_{1}}{2} .
$$

By Lemma 9.5 for any $\tau \in\left[\tau_{*}, \tau_{2}(r)\right]$ it is

$$
\Psi_{z}(\tau) \leq \Psi_{z}\left(\tau_{*}\right)+L_{1} \tau_{2}(r) \sqrt{2 M_{0} L_{0}}<\frac{c_{1}}{2}+L_{1} \tau_{2}(r) \sqrt{2 M_{0} L_{0}} \leq \frac{3}{4} c_{1},
$$

provided that

$$
L_{1} \tau_{2}(r) \sqrt{2 M_{0} L_{0}} \leq \frac{1}{4} c_{1} .
$$

Note that the same estimate made in (9.61) allows to treat also the case

$$
\exists i \in\{1, \ldots, k\} \text { and } \tau_{*} \in\left[0, \tau_{2}(r)\right] \text { such that }\left[a_{\tau_{*}}, b_{\tau_{*}}\right] \cap\left[a_{x_{i}}, b_{x_{i}}\right]=\emptyset,
$$

where $\left[a_{x_{i}}, b_{x_{i}}\right] \in \mathcal{I}_{x_{i}, r, \varepsilon}$ is the interval satisfying (9.57), as done in Step 6 to prove (9.51).

Now suppose that

there exist $\tau_{*} \in\left[0, \tau_{2}(r)\right]$ and $i \in\{1, \ldots, k\}$ such that: $\Psi_{z}\left(\tau_{*}\right) \geq \frac{c_{1}}{2}$ and

$$
\begin{aligned}
{\left[a_{\tau_{*}}, b_{\tau_{*}}\right] \cap\left[a_{x_{i}}, b_{x_{i}}\right] } & \neq \emptyset \text { and } D\left(x_{i}, a_{\tau_{*}}, a_{\tau_{*}}, a_{x_{i}}, b_{x_{i}}\right)>E(r) \\
\text { or }\left[a_{\tau_{*}}, b_{\tau_{*}}\right] \cap\left[a_{\mathcal{R} x_{i}}, b_{\mathcal{R} x_{i}}\right] & \neq \emptyset \text { and } D\left(\mathcal{R} x_{i}, a_{\tau_{*}}, a_{\tau_{*}}, a_{\mathcal{R} x_{i}}, b_{\mathcal{R} x_{i}}\right)>E(r),
\end{aligned}
$$

where $\left[a_{x_{i}}, b_{x_{i}}\right]$ is the interval satisfying (9.57).

In this case by Lemma 9.2 with $K_{*}=E(r)$ we deduce that

$$
\Psi_{z}\left(\tau_{*}\right) \leq \Psi_{z}(0)-\frac{c_{1} E(r)}{32 M_{0}} .
$$

Then, by Lemma 9.5 for any $\tau \in\left[\tau_{*}, \tau_{2}(r)\right]$ it is

$$
\begin{array}{r}
\Psi_{z}(\tau) \leq \Psi_{z}\left(\tau_{*}\right)+L_{1} \tau_{2}(r) \sqrt{2 M_{0} L_{0}} \leq \Psi_{z}(0)-\frac{c_{1} E(r)}{32 M_{0}}+L_{1} \tau_{2}(r) \sqrt{2 M_{0} L_{0}} \leq \\
\Psi_{z}(0)-\frac{c_{1} E(r)}{64 M_{0}},
\end{array}
$$

provided that

$$
L_{1} \tau_{2}(r) \sqrt{2 M_{0} L_{0}} \leq \frac{c_{1} E(r)}{64 M_{0}} .
$$

Then it remains to consider only the case:

for any $\tau \in\left[0, \tau_{2}(r)\right]$, for any $i \in\{1, \ldots, k\}$ it is:

$$
\begin{aligned}
& \Psi_{z}(\tau) \geq \frac{c_{1}}{2},\left[a_{\tau}, b_{\tau}\right] \cap\left[a_{x_{i}}, b_{x_{i}}\right] \neq \emptyset,\left[a_{\tau}, b_{\tau}\right] \cap\left[a_{\mathcal{R} x_{i}}, b_{\mathcal{R} x_{i}}\right] \neq \emptyset, \\
& D\left(x_{i}, a_{\tau_{*}}, b_{\tau_{*}}, a_{x_{i}}, b_{x_{i}}\right) \leq E(r) \text { and } D\left(\mathcal{R} x_{i}, a_{\tau_{*}}, b_{\tau_{*}}, a_{\mathcal{R} x_{i}}, b_{\mathcal{R} x_{i}}\right) \leq E(r), \\
& \text { where }\left[a_{x_{i}}, b_{x_{i}}\right] \in \mathcal{I}_{x_{i}, r, \varepsilon} \text { satisfies }(9.57) .
\end{aligned}
$$


Now recall that $V_{x_{i}}$ has been chosen so that $\left\|V_{x_{i}}\right\|_{a_{x_{i}}, b_{x_{i}}}=\frac{1}{2}$ for any $i$, while $c_{1} \geq$ $\frac{1}{2}\left(\frac{3 \delta_{0}}{4 K_{0}}\right)^{2}$ so $\frac{c_{1}}{2} \geq \frac{1}{4}\left(\frac{3 \delta_{0}}{4 K_{0}}\right)^{2}$. Then by the choice of $\rho\left(x_{i}\right),(9.60)$, Steps 5 and 6 and Propositions 6.1 and 6.4 we have:

$$
\begin{aligned}
\limsup _{l \rightarrow 0^{+}} \frac{\Psi_{z}(\tau+l)-\Psi_{z}(\tau)}{l} \leq \\
\quad\left(b_{\tau}-a_{\tau}\right) \sum_{i=1}^{k}\left(\beta_{x_{i}}(z)\left(-\frac{\mu(r)}{4}\right)+\beta_{\mathcal{R} x_{i}}(z)\left(-\frac{\mu(r)}{4}\right)\right)+ \\
\quad \lambda\left(b_{\tau}-a_{\tau}\right) N_{0} \int_{a_{\tau}}^{b_{\tau}} g\left(\dot{y}_{\tau}, \dot{y}_{\tau}\right) d s \leq\left(b_{\tau}-a_{\tau}\right)\left(-\frac{\mu(r)}{4}\right)+2 \lambda N_{0} M_{0} .
\end{aligned}
$$

Now $b_{\tau}-a_{\tau} \geq \frac{c_{1}}{2 M_{0}}$, therefore

$$
\limsup _{l \rightarrow 0^{+}} \frac{\Psi_{z}(\tau+l)-\Psi_{z}(\tau)}{l} \leq-\frac{c_{1}}{2 M_{0}} \frac{\mu(r)}{4}+2 \lambda N_{0} M_{0} \leq-\frac{\mu(r) c_{1}}{16 M_{0}}
$$

choosing $\lambda_{2}$ such that $2 \lambda_{2} N_{0} M_{0} \leq \frac{\mu(r) c_{1}}{16 M_{0}}$.

We can finally conclude the proof of Proposition 9.1 defining

$$
H_{\varepsilon}(\tau, z)=\eta\left(\tau T_{\varepsilon}, z\right)
$$

which is of type $A$ in $\left.\left.[0,1], T_{\varepsilon}=2 \frac{\varepsilon 16 M_{0}}{c_{1} \mu(r)}, \varepsilon \in\right] 0, \varepsilon_{1}(r)\right]$, and $\varepsilon_{1}(r)$ such that $T_{\varepsilon_{1}(r)} \leq$ $\tau_{2}(r)$.

By Steps 1,2,4 and 6, $\left(\mathcal{D}, H_{\varepsilon} \star h\right) \in \mathcal{H}$, so (11) of Proposition 9.1 is satisfied. By Step 7 it follows that also (2) of Proposition 9.1 is satisfied. Finally property (9.46) gives (3).

\section{DEFORMATION RESUlTS FOR HOMOTOPIES OF TYPE B}

In this section we study how to proceed when there are curves nearby variationally critical portions of second type. On these curves we use a suitable reparameterization flow to push down the functional $\mathcal{F}$. The construction of the reparameterizations is based on the following simple idea. Fix a curve $x:[a, b] \rightarrow H^{1}\left([a, b], \mathbb{R}^{N}\right)$ and fix $\left.c \in\right] a, b[$. Consider the integral average of the energy in the intervals $[a, c]$ and $[c, b]$. Suppose that they are different and take an affine reparameterization of $[a, c]$ on $[a, c+\tau]$ and of $[c, b]$ on $[c+\tau, b]$. In such a way we can move $c$ towards $a$ if $\tau<0$ and $c$ towards $b$ if $\tau>0$, and consider the corresponding reparameterization $x_{\tau}$ of the curve $x$. Moving $c$ towards the extreme point of the interval where the integral average is smaller, the energy functional decreases. This fact is rigorously explained in Remark 10.2.

The above property is clearly satisfied for any irregular variationally critical portion $x$ of II type if we choose as $c$ an instant nearby an interval where $x$ is constant. We can therefore to push down $\mathcal{F}$ in a neighborhood of any irregular variationally critical portion of II type, obtaining Proposition 10.3. We shall do this trying to follow as much as possible the frame of the previous section.

Note that using reparameterizations there is the possibility that pieces of curves outside $\Omega$ move inside $\Omega$. Anyway this is allowed for homotopies of type B and it is not a problem since we are just dealing with reparametrization of intervals.

Let $x \in \mathfrak{M}$ be fixed; for all $[a, b] \in \mathcal{I}_{x}$ such that $x_{\mid[a, b]}$ is an irregular variationally critical portion of second type, we set:

$$
\begin{aligned}
& \ell_{-}(x)=\max \{s \in] 0, b-a\left[: x_{\mid[a, a+s]} \text { is constant }\right\}, \\
& \ell_{+}(x)=\max \{s \in] 0, b-a\left[: x_{\mid[b-s, b]} \text { is constant }\right\} .
\end{aligned}
$$


Remark 10.1. Note that, by Proposition 4.2 if $x_{\mid[a, b]}$ is of first type, it may be $\ell_{-}(x)+$ $\ell_{+}(x)=0$, while if $x_{\mid[a, b]}$ is of second type we have $\ell_{-}(x)+\ell(x)>0$, and such a quantity is, in a sense, a measure of the distance from $x_{\mid[a, b]}$ to the set of OGC's affinely reparameterized in $\left[a+\ell_{-}(x), b-\ell_{+}(x)\right]$.

Instead of considering only reparameterization flows we prefer to give the analogous of Proposition 9.1 when we are far only from regular variationally critical portions and from irregular variationally critical portions of first type. Nearby irregular variationally critical portion of II type we use reparameteriztions of the type described in Remark 10.2, while far from them we use the results of Proposition 9.1.

To this aim, we set:

$$
\begin{aligned}
\mathcal{Z}_{a, b}^{1}=\left\{y \in H^{1}([a, b], \bar{\Omega})\right. & : \text { either } y_{\mid[a, b]} \text { is an OGC }, \\
& \text { or } \left.y_{\mid[a, b]} \text { is an irregular variationally critical portion of first type. }\right\}
\end{aligned}
$$

Remark 10.2. Assume $[a, b] \subset[0,1]$ and let $c \in] 0,1\left[\right.$ be fixed. Let $\tau_{0}>0$ be such that $c+\tau \in] a, b[$ for all $\tau \in]-\tau_{0}, \tau_{0}\left[\right.$, and for all $x \in \mathfrak{M}$ denote by $x_{a, c, \tau}$ the (orientation preserving) affine reparameterization on the interval $[a, c+\tau]$ of $x_{\mid[a, c]}$. Similarly, define $x_{c, \tau, b}$ to be the affine reparameterization of $x_{[[c, b]}$ on $[c+\tau, b]$. Set:

$$
\varphi(\tau)=\frac{1}{2} \int_{a}^{c+\tau} g\left(\dot{x}_{a, c, \tau}, \dot{x}_{a, c, \tau}\right) \mathrm{d} s+\int_{c+\tau}^{b} g\left(\dot{x}_{c, \tau, b}, \dot{x}_{c, \tau, b}\right) \mathrm{d} s .
$$

A straightforward calculation gives:

$$
\varphi(\tau)=\frac{c-a}{2(c-a+\tau)} \int_{a}^{c} g(\dot{x}, \dot{x}) \mathrm{d} s+\frac{b-c}{2(b-c-\tau)} \int_{c}^{b} g(\dot{x}, \dot{x}) \mathrm{d} s .
$$

Hence,

$$
\varphi^{\prime}(\tau)=-\frac{(c-a) \int_{a}^{c} g(\dot{x}, \dot{x}) \mathrm{d} s}{2(c-a+\tau)^{2}}+\frac{(b-c) \int_{c}^{b} g(\dot{x}, \dot{x}) \mathrm{d} s}{2(b-c-\tau)^{2}},
$$

and in particular:

$$
\varphi^{\prime}(0)=-\frac{1}{2(c-a)} \int_{a}^{c} g(\dot{x}, \dot{x}) \mathrm{d} s+\frac{1}{2(b-c)} \int_{c}^{b} g(\dot{x}, \dot{x}) \mathrm{d} s .
$$

Proposition 10.3. Fix $c_{1} \in\left[\frac{1}{2}\left(\frac{3 \delta_{0}}{4 K_{0}}\right)^{2}, M_{0}\left[\right.\right.$ and $r>0$. Then, there exists $\varepsilon_{2}=\varepsilon_{2}\left(r, c_{1}\right) \in$ ] $0, \frac{c_{1}}{4}[$ such that for any $\left.\varepsilon \in] 0, \varepsilon_{2}\right]$, for any $(\mathcal{D}, h) \in \mathcal{H}$ satisfying:

$$
\mathcal{F}(\mathcal{D}, h) \leq c_{1}+\varepsilon
$$

and

$$
\begin{aligned}
& \left\|y-x_{\mid[a, b]}\right\|_{a, b} \geq r \\
& \qquad \text { for any } y \in \mathcal{Z}_{a, b}^{1}, \text { for any } x=h(1, \xi), \xi \in \mathcal{D} \text {, such that } \\
& \qquad \frac{b-a}{2} \int_{a}^{b} g(\dot{x}, \dot{x}) \mathrm{d} s \geq c_{1}-\varepsilon \text {, where }[a, b] \in \mathcal{I}_{x} .
\end{aligned}
$$

there exists a continuous map $H_{\varepsilon}:[0,1] \times h(1, \mathcal{D}) \rightarrow \mathfrak{M}$, concatenation of a homotopy of type $A$ in $[0,1]$ with another of type $B$, with the following properties:

(1) $\left(\mathcal{D}, H_{\varepsilon} \star h\right) \in \mathcal{H}$;

(2) $\mathcal{F}\left(\mathcal{D}, H_{\varepsilon} \star h\right) \leq c_{1}-\varepsilon$;

(3) there exists $T_{\varepsilon}>0$, with $T_{\varepsilon} \rightarrow 0$ as $\varepsilon \rightarrow 0$, such that for any $z \in h(1, \mathcal{D})$ $\left\|H_{\varepsilon}(\tau, z)-z\right\|_{a, b} \leq \tau T_{\varepsilon}$ for all $\tau \in[0,1]$, for all $[a, b] \in \mathcal{I}_{z}$. 
In order to prove Proposition 10.3 some preliminary results are needed. Thanks to Remark 4.7 a simple contradiction argument gives

Lemma 10.4. There exist $\rho_{2}>0$ and $\ell>0$ such that, if $x \in \mathfrak{M},[a, b] \in \mathcal{I}_{x}$ and

$$
\left\|x_{\mid[a, b]}-y\right\|_{a, b} \geq r \text { for any } y \in Z_{a, b}^{1},
$$

then

$$
\begin{gathered}
\ell_{-}(w)+\ell_{+}(w) \geq \ell, \\
\frac{1}{2} \int_{a}^{b} g(\dot{w}, \dot{w}) \mathrm{d} s \leq M_{0}+1,
\end{gathered}
$$

for any $w_{\mid[a, b]}$ irregular variational portion of II type satisfying $\left\|x_{\mid[a, b]}-w_{\mid[a, b]}\right\|_{a, b} \leq \rho_{2}$.

Now we have the following

Lemma 10.5. Let $w_{\mid[a, b]}$ be an irregular critical variational portion of second type satisfying (10.10) and

$$
\ell_{-}(w) \geq \frac{\ell}{2}
$$

Then there exist $\theta(\ell)>0, \mu(\ell)>0$ and $\delta(\ell)>0$ such that, setting

$$
\left.c_{w}=a+\ell_{-}(w)+\theta(\ell) \in\right] a, b[
$$

we have

$$
\frac{1}{2\left(c_{w}-a\right)} \int_{a}^{c_{w}} g(\dot{w}, \dot{w}) \mathrm{d} s-\frac{1}{2\left(b-c_{w}\right)} \int_{c_{w}}^{b} g(\dot{w}, \dot{w}) \mathrm{d} s \leq-2 \mu(\ell) .
$$

(The case $\ell_{+}(w) \geq \frac{\ell}{2}$ is completely analogous).

Proof. Denote by $E_{w}$ the energy of the OGC $w$ on the interval $\left[a+\ell_{-}(w), b-\ell_{+}(w)\right]$, so that

$$
g(\dot{w}(s), \dot{w}(s))=E_{w} \text { for any } s \in\left[a+\ell_{-}(w), b-\ell_{+}(w)\right] .
$$

Now set $c_{w}=a+\ell_{-}(w)+\theta$ with $\theta>0$. Since $w$ is constant on $[a, b] \backslash\left[a+\ell_{-}(w), b-\right.$ $\left.\ell_{+}(w)\right]$ it is

$$
\begin{gathered}
\frac{1}{2\left(c_{w}-a\right)} \int_{a}^{c_{w}} g(\dot{w}, \dot{w}) \mathrm{d} s-\frac{1}{2\left(b-c_{w}\right)} \int_{c_{w}}^{b} g(\dot{w}, \dot{w}) \mathrm{d} s= \\
{\left[\frac{\theta}{2\left(c_{w}-a\right)}+\frac{\theta}{2\left(b-c_{w}\right)}-\frac{1}{2\left(b-c_{w}\right)}\left((b-a)-\left(\ell_{-}(w)+\ell_{+}(w)\right)\right] E_{w} \leq\right.} \\
{\left[\frac{\theta}{\ell}+\frac{\theta}{2\left(b-c_{w}\right)}-\frac{1}{2}\left((b-a)-\left(\ell_{-}(w)+\ell_{+}(w)\right)\right] E_{w}\right.}
\end{gathered}
$$

since $\left(b-c_{w}\right) \leq 1$ (recall that $\left.\left[c_{w}, b\right] \subset[0,1]\right)$ and $c_{w}-a \geq \ell_{-}(w) \geq \frac{\ell}{2}$.

Now $\left((b-a)-\left(\ell_{-}(w)+\ell_{+}(w)\right)\right)$ is the length of the interval $\left[a+\ell_{-}(w), b-\ell_{+}(w)\right]$ where $w$ is an OGC of energy $E_{w}$. Since $\frac{1}{2} \int_{a}^{b} g(\dot{w}, \dot{w}) \leq M_{0}+1$ the strong concavity assumption (1.7) and Lemma 2.1 imply that

$$
(b-a)-\left(\ell_{-}(w)+\ell_{+}(w)\right) \geq \frac{\delta_{0}^{2}}{2 K_{0}^{2}\left(M_{0}+1\right)}
$$

and

$$
E_{w} \geq \frac{\delta_{0}^{2}}{K_{0}^{2}}
$$

because $b-a \leq 1$. 
Now $b-c_{w} \geq(b-a)-\left(\ell_{-}(w)+\ell_{+}(w)\right)-\theta$ and $c_{w}-a \geq \frac{\ell}{2}$, therefore we can choose $\theta=\theta(\ell)$ (independently of $w$ ) sufficiently small such that

$$
\begin{aligned}
\frac{1}{2\left(c_{w}-a\right)} \int_{a}^{c_{w}} g(\dot{w}, \dot{w}) \mathrm{d} s-\frac{1}{2\left(b-c_{w}\right)} \int_{c_{w}}^{b} g(\dot{w}, \dot{w}) \mathrm{d} s \leq \\
\left(\frac{\theta}{\ell}+\frac{1}{2} \frac{\theta}{\frac{\delta_{0}^{2}}{2 K_{0}^{2}\left(M_{0}+1\right)}-\theta}-\frac{1}{2} \frac{\delta_{0}^{2}}{2 K_{0}^{2}\left(M_{0}+1\right)}\right) \frac{\delta_{0}^{2}}{K_{0}^{2}} \leq-2 \mu(\ell),
\end{aligned}
$$

where $\mu(\ell)>0$.

Now, by Remark 4.7 and Lemma 10.5 we immediately obtain the following

Lemma 10.6. There exist $\left.\rho(\ell) \in] 0, \rho_{2}\right]$ such that for any $x \in h(1, \mathcal{D})$ and $[a, b] \in \mathcal{I}_{x}$, if there exists $w_{\mid[a, b]}$ irregular variationally critical portion of II type such that

$$
\|x-w\|_{a, b} \leq \rho(\ell), \text { and } \ell_{-}(w) \geq \frac{\ell}{2},
$$

there exists $\left.c_{x} \in\right] a, b[$ such that

$$
\frac{1}{2\left(c_{x}-a\right)} \int_{a}^{c_{x}} g(\dot{x}, \dot{x}) \mathrm{d} s-\frac{1}{2\left(b-c_{x}\right)} \int_{c_{x}}^{b} g(\dot{x}, \dot{x}) \mathrm{d} s \leq-\mu(\ell) .
$$

Let $\rho(\ell)$ be as in Lemma 10.6. We can state the analogous of Proposition 6.1 as follows. Set

$$
\mathcal{V}_{\ell}^{-}=\left\{x \in h(1, \mathcal{D}): \exists\left[a_{x}, b_{x}\right] \in \mathcal{I}_{x}\right.
$$

and $w_{\mid\left[a_{x}, b_{x}\right]}$ irregular variationally critical portion of II type satisfying

$$
\left.\ell_{-}(w) \geq \frac{\ell}{2} \text { and }\|x-w\|_{a_{x}, b_{x}}<\rho(\ell)\right\} .
$$

Let $x \in \mathcal{V}_{\ell}^{-}$. We shall set

$$
\begin{array}{r}
\mathcal{I}_{x, \rho(\ell)}=\left\{\left[a_{x}, b_{x}\right] \in \mathcal{I}_{x}: \exists w_{\mid\left[a_{x}, b_{x}\right]}\right. \text { irregular variationally critical portion of II type } \\
\text { satisfying } \left.\ell_{-}(w) \geq \frac{\ell}{2} \text { and }\|x-w\|_{a_{x}, b_{x}}<\rho(\ell)\right\} .
\end{array}
$$

Let $\left[a_{x}, b_{x}\right] \in \mathcal{I}_{x, \rho(\ell)}$ and $\left.c_{x} \in\right] a_{x}, b_{x}[$ given by Lemma 10.6. Define for any $\tau \in] 0, c_{x}-a_{x}[$ and $s \in[0,1]$ :

(10.15)

$$
\varphi_{x, a_{x}, c_{x}, b_{x},-}(\tau, s) \equiv \varphi_{x,-}(\tau, s)= \begin{cases}s, & \text { if } s \in\left[0, a_{x}\right] \\ \frac{c_{x}-a_{x}}{c_{x}-\tau-a_{x}}\left(s-a_{x}\right)+a_{x}, & \text { if } s \in\left[a_{x}, c_{x}-\tau\right] \\ \frac{b_{x}-c_{x}}{b_{x}+\tau-c_{x}}\left(s-c_{x}+\tau\right)+c_{x}, & \text { if } s \in\left[c_{x}-\tau, b_{x}\right] \\ s, & \text { if } s \in\left[b_{x}, 1\right] .\end{cases}
$$

if $x \in \mathcal{V}_{\ell}^{+}$, we choose $c_{x}$ starting from $b-\ell_{+}-\theta(\ell)$ and define

$$
\varphi_{x, a_{x}, c_{x}, b_{x},+}(\tau, s) \equiv \varphi_{x,+}(\tau, s)= \begin{cases}s, & \text { if } s \in\left[0, a_{x}\right] \\ \frac{c_{x}-a_{x}}{c_{x}+\tau-a_{x}}\left(s-a_{x}\right)+a_{x}, & \text { if } s \in\left[a_{x}, c_{x}+\tau\right] \\ \frac{b_{x}-c_{x}}{b_{x}-\tau-c_{x}}\left(s-c_{x}-\tau\right)+c_{x}, & \text { if } s \in\left[c_{x}+\tau, b_{x}\right] \\ s, & \text { if } s \in\left[b_{x}, 1\right] .\end{cases}
$$


By Lemma 10.6 a straightforward computation (made in $\tau=0$ ) gives the following result, (analogous of Proposition 6.1).

Proposition 10.7. Let $\mu(\ell)$ and $\theta(\ell)$ be as in Lemma 10.5. There exists $\tau(\ell) \in] 0, \theta(\ell)]$ such that, for any $\tau \in[0, \tau(\ell)]$, for any $x \in \mathcal{V}_{\ell}^{-}$and for any $\left[a_{x}, b_{x}\right]$ as in 10.14 we have:

$$
\int_{a_{x}}^{b_{x}} g(\dot{x}(\sigma), \dot{x}(\sigma)) \frac{\partial^{2} \varphi_{x,-}}{\partial \tau \partial s}\left(\tau, \varphi_{x,-}^{-1}(\tau, \sigma)\right) d \sigma \leq-\frac{3 \mu(\ell)}{4} .
$$

Remark 10.8. An analogous results clearly holds for $x \in \mathcal{V}_{\ell}^{+}$using the parameterization $\varphi_{x,+} \cdot$

Observe also that

$$
x \in \mathcal{V}_{\ell}^{-} \Longleftrightarrow \mathcal{R} x \in \mathcal{V}_{\ell}^{+}
$$

and we choose

$$
\varphi_{\mathcal{R} x, 1-b_{x}, 1-c_{x}, 1-a_{x},+}(\tau, s)=\varphi_{x, a_{x}, c_{x}, b_{x},-}(\tau, s) .
$$

By $\Phi_{x}$ we shall denote the map $\varphi_{x,-}$ if $x \in \mathcal{V}_{\ell}^{-} \backslash \mathcal{V}_{\ell}^{+}$and the map $\varphi_{x,+}$ if $x \in \mathcal{V}_{\ell}^{+} \backslash \mathcal{V}_{\ell}^{-}$, relatively to $\left[a_{x}, b_{x}\right]$. Whenever $x \in \mathcal{V}_{\ell}^{-} \cap \mathcal{V}_{\ell}^{+}$we shall denote by $\Phi_{x}$ just one of the two maps above. It will be only important to obey to property (10.16) to have $\mathcal{R}$-invariance.

Now we state and proof the analogous of Proposition 6.4, whenever $x \in \mathcal{V}_{\ell}^{-}$(the case $x \in \mathcal{V}_{\ell}^{+}$being completely analogous). Let $\mu(\ell)$ given by Lemma 10.5 .

Proposition 10.9. There exists $\left.\left.\left.\left.\tau_{1}(\ell) \in\right] 0, \tau(\ell)\right], \rho_{1}(\ell) \in\right] 0, \min (1, \rho(\ell))\right]$ and $E_{1}(\ell)>0$ such that for any $x \in \mathcal{V}_{\ell}^{-}$and $\left[a_{x}, b_{x}\right] a$ in (10.14), if $\varphi_{x,-}$ is the parameterization (10.15), the following property holds:

$$
\begin{aligned}
& \text { for any } z \in h(1, \mathcal{D}) \text {, and for all }\left[a_{z}, b_{z}\right] \in \mathcal{I}_{z} \text { such that } \\
& \qquad \begin{array}{r}
\|x-z\|_{a_{z}, b_{z}}<\rho_{1}(\ell),\left[a_{z}, b_{z}\right] \cap\left[a_{x}, b_{x}\right] \neq \emptyset \text { and } D\left(x, a_{z}, b_{z}, a_{x}, b_{x}\right) \leq E_{1}(\ell) \text { it is } \\
\qquad \int_{a_{z}}^{b_{z}} g(\dot{z}(\sigma), \dot{z}(\sigma)) \frac{\partial^{2} \varphi_{x,-}}{\partial \tau \partial s}\left(\tau, \varphi_{x,-}^{-1}(\tau, \sigma)\right) d \sigma \leq-\frac{\mu(\ell)}{2} \text { for any } \tau \in\left[0, \tau_{1}(\ell)\right] .
\end{array}
\end{aligned}
$$

Proof. First note that there exists $M(\ell)>0$ such that

$$
\left|\frac{\partial^{2} \varphi_{x,-}}{\partial \tau \partial s}\left(\tau, \varphi_{x,-}^{-1}(\tau, s)\right)\right| \leq M(\ell) \text { for any } \tau \in[0, \tau(\ell)] \text {, for any } s \in[0,1] .
$$

Then, the same estimate used to prove 9.13 ) allows to obtain

$$
\begin{aligned}
\mid \int_{a_{z}}^{b_{z}} g(\dot{z}(\sigma), \dot{z}(\sigma)) & \frac{\partial^{2} \varphi_{x,-}}{\partial \tau \partial s}\left(\tau, \varphi_{x,-}^{-1}(\tau, \sigma)\right) d \sigma- \\
& \int_{a_{z}}^{b_{z}} g(\dot{x}(\sigma), \dot{x}(\sigma)) \frac{\partial^{2} \varphi_{x,-}}{\partial \tau \partial s}\left(\tau, \varphi_{x,-}^{-1}(\tau, \sigma)\right) d \sigma \mid \leq \widehat{K} M(\ell) \rho_{1}(\ell) .
\end{aligned}
$$

where $\widehat{K}$ is defined by (9.4). Finally

$$
\begin{gathered}
\mid \int_{a_{z}}^{b_{z}} g(\dot{x}(\sigma), \dot{x}(\sigma)) \frac{\partial^{2} \varphi_{x,-}}{\partial \tau \partial s}\left(\tau, \varphi_{x,-}^{-1}(\tau, \sigma)\right) d \sigma- \\
\int_{a_{x}}^{b_{x}} g(\dot{x}(\sigma), \dot{x}(\sigma)) \frac{\partial^{2} \varphi_{x,-}}{\partial \tau \partial s}\left(\tau, \varphi_{x,-}^{-1}(\tau, \sigma)\right) d \sigma \mid \leq \\
M(\ell) \int_{I_{a_{z}, a_{x}}} g(\dot{x}, \dot{x}) \mathrm{d} s+M(\ell) \int_{I_{b_{z}, b_{x}}} g(\dot{x}, \dot{x}) \mathrm{d} s \leq \\
2 M(\ell) D\left(x, a_{z}, b_{z}, a_{x}, b_{x}\right) \leq 2 M(\ell) E_{1}(\ell) .
\end{gathered}
$$

Then, if $E_{1}(\ell)$ is sufficiently small (depending only by $\ell$ ) by Proposition 10.7 we have the thesis. 
Proof of Proposition 10.3. For sake of simplicity we shall assume that any $x \in \mathcal{V}_{\ell}^{-} \cup \mathcal{V}_{\ell}^{+}$ has only one interval $\left[a_{x}, b_{x}\right] \in \mathcal{I}_{x, \rho(\ell)}$. Indeed the general case can be carried on by small changes.

Starting from any $z \in \overline{\mathcal{V}_{\ell}^{-}} \cup \overline{\mathcal{V}_{\ell}^{+}}$, we apply the reparameterization flow

$$
z_{\tau}(s)=z\left(\Phi_{z}(\tau, s)\right)
$$

where

$$
\Phi_{z}(\tau, s)=\sum_{j=1, \ldots, k}\left(\beta_{x_{j}}(z) \Phi_{x_{j}}(\tau, s)+\beta_{\mathcal{R} x_{j}}(z) \Phi_{\mathcal{R} x_{j}}(\tau, s)\right),
$$

$\left\{\beta_{x_{j}}, \beta_{\mathcal{R} x_{j}}\right\}$ is a partition of the unity (defined on $\overline{\mathcal{V}_{\ell}^{-}} \cup \overline{\mathcal{V}_{\ell}^{+}}$), as that one used in the proof of Proposition 9.1, and $\Phi_{x_{j}}$ is described in Remark 10.8. The partition of the unity is subordinated to the open finite covering $\left\{\mathcal{U}_{x_{i}}, \mathcal{U}_{\mathcal{R} x_{i}}\right\}$ where $\mathcal{U}_{x_{i}}=\left\{z \in \overline{\mathcal{V}_{\ell}^{-}} \cup \overline{\mathcal{V}_{\ell}^{+}}\right.$: $\left.\left\|z-x_{i}\right\|_{0,1}<\rho_{1}\left(x_{i}\right)\right\}$, with $\rho_{1}(x)<\rho_{1}(\ell)$ (where $x \in \overline{\mathcal{V}_{\ell}^{-}} \cup \overline{\mathcal{V}_{\ell}^{+}}$) that will be chosen later.

Fix $\left[a_{z}, b_{z}\right] \in \mathcal{I}_{z}, z \in \overline{\mathcal{V}_{\ell}^{-}} \cup \overline{\mathcal{V}_{\ell}^{+}}$and set

$$
\Gamma_{z}(\tau)=\int_{a_{\tau}}^{b_{\tau}} g\left(\dot{z}_{\tau}, \dot{z}_{\tau}\right) \mathrm{d} s
$$

where $z_{\tau}$ is defined in 10.17), $a_{\tau}=\Phi_{z}\left(\tau, a_{z}\right)$ and $b_{\tau}=\Phi_{z}\left(\tau, b_{z}\right)$. Since $\Phi_{z}(0, s)=s$ for any $s \in[0,1]$, we have $z_{0}=z$, while

$$
\dot{z}_{\tau}(s)=z\left(\Phi_{z}(\tau, s)\right) \frac{\partial \Phi_{z}}{\partial s}(\tau, s)
$$

and

$$
\Gamma_{z}(\tau)=\int_{\Phi_{z}\left(\tau, a_{z}\right)}^{\Phi_{z}\left(\tau, b_{z}\right)} g\left(\dot{z}\left(\Phi_{z}(\tau, s)\right), \dot{z}\left(\Phi_{z}(\tau, s)\right)\left(\frac{\partial \Phi_{z}}{\partial s}(\tau, s)\right)^{2} \mathrm{~d} s .\right.
$$

Note that, since $s \mapsto \Phi_{z}(\tau, s)$ is strictly increasing, the interval $\left[\Phi_{z}\left(\tau, a_{z}\right), \Phi_{z}\left(\tau, b_{z}\right)\right] \in$ $\mathcal{I}_{z_{\tau}}$, while setting $\Phi_{z}(\tau, s)=\sigma$ we have

$$
\begin{aligned}
\Gamma_{z}(\tau)=\int_{a_{z}}^{b_{z}} g(\dot{z}(\sigma), \dot{z}(\sigma)) \frac{\partial \Phi_{z}}{\partial s}(\tau, s) d \sigma= \\
\sum_{j=1, \ldots, k} \beta_{x_{j}}(z) \int_{a_{z}}^{b_{z}} g(\dot{z}(\sigma), \dot{z}(\sigma)) \frac{\partial \Phi_{x_{j}}}{\partial s}\left(\tau, \Phi_{x_{j}}^{-1}(\tau, \sigma)\right) d \sigma \\
\quad+\sum_{j=1, \ldots, k} \beta_{\mathcal{R} x_{j}}(z) \int_{a_{z}}^{b_{z}} g(\dot{z}(\sigma), \dot{z}(\sigma)) \frac{\partial \Phi_{\mathcal{R} x_{j}}}{\partial s}\left(\tau, \Phi_{\mathcal{R} x_{j}}^{-1}(\tau, \sigma)\right) d \sigma .
\end{aligned}
$$

Then by Proposition 10.9, since $\sum_{j=1, \ldots, k}\left(\beta_{x_{j}}(z)+\beta_{\mathcal{R} x_{j}}(z)\right)=1$, if

(10.18) $\left[a_{z}, b_{z}\right] \cap\left[a_{x_{i}}, b_{x_{i}}\right] \neq \emptyset$,

$$
D\left(x_{i}, a_{z}, b_{z}, a_{x_{i}}, b_{x_{i}}\right) \leq E_{1}(\ell) \text { and } D\left(\mathcal{R} x_{i}, a_{z}, b_{z}, a_{\mathcal{R} x_{i}}, b_{\mathcal{R} x_{i}}\right) \leq E_{1}(\ell) \text { for any } i
$$

we have

$$
\frac{\partial \Gamma_{z}}{\partial \tau}(\tau) \leq-\frac{\mu(\ell)}{2} \text { for any } \tau \in\left[0, \tau_{1}(\ell)\right]
$$

Note that, (analogously to the proof of Proposition 9.1) we can choose $\rho_{1}(x)$ sufficiently small so that if, for some $i,\left[a_{z}, b_{z}\right] \cap\left[a_{x_{i}}, b_{x_{i}}\right]=\emptyset$ and $a_{z}$ is close to $a_{x_{i}}$ or $b_{z}$ is close to $b_{x_{i}}$, then the energy $\frac{1}{2} \int_{a_{z}}^{b_{z}} g(\dot{z}, \dot{z}) \mathrm{d} s$ is small. Then using the analogous of Lemmas 9.2 and 9.5 , from 10.19 we can deduce that

$$
\left.\left.z_{\tau} \in \mathfrak{M} \forall \tau \in\left[0, \tau_{2}(\ell)\right], \text { for some } \tau_{2}(\ell) \in\right] 0, \tau_{1}(\ell)\right] .
$$


Then, to conclude the proof it remains to study the

$$
\Delta_{z}(\tau)=\left(b_{\tau}-a_{\tau}\right) \Gamma_{z}(\tau) .
$$

Since $b_{\tau}-a_{\tau}$ is not necessarily decreasing (as we deduce calculating the derivative with respect to $\tau$ of $\varphi_{x_{i},-}$ and $\left.\varphi_{x_{i},+}\right)$ it is convenient to recall that

$$
\Gamma_{z}(\tau)<M_{0} \text { for any } \tau \in\left[0, \tau_{1}(\ell)\right]
$$

and observe that

$$
\begin{aligned}
b_{\tau}-a_{\tau}= & \sum_{j=1, \ldots, k} \beta_{x_{j}}(z)\left[\Phi_{x_{j}}\left(\tau, b_{z}\right)-\Phi_{x_{j}}\left(\tau, a_{z}\right)\right]+ \\
& \sum_{j=1, \ldots, k} \beta_{\mathcal{R} x_{j}}(z)\left[\Phi_{\mathcal{R} x_{j}}\left(\tau, b_{z}\right)-\Phi_{\mathcal{R} x_{j}}\left(\tau, a_{z}\right)\right] .
\end{aligned}
$$

Now for any $x$ it is

$$
\frac{\partial \varphi_{x,-}}{\partial \tau}(\tau, s)= \begin{cases}0, & \text { if } s \in\left[0, a_{x}\right] \\ \frac{c_{x}-a_{x}}{\left(c_{x}-\tau-a_{x}\right)^{2}}\left(s-a_{x}\right), & \text { if } s \in\left[a_{x}, c_{x}-\tau\right] \\ \frac{b_{x}-c_{x}}{\left(b_{x}+\tau-c_{x}\right)^{2}}\left(b_{x}-s\right), & \text { if } s \in\left[c_{x}-\tau, b_{x}\right] \\ 0, & \text { if } s \in\left[b_{x}, 1\right]\end{cases}
$$

and analogously for $\varphi_{x,+}$. Then by 10.19), we can deduce the existence of $\sigma(\ell)>0$ and $\left.\left.\tau_{3}(\ell) \in\right] 0, \tau_{2}(\ell)\right]$ such that

$$
\begin{gathered}
a_{z}<a_{x_{j}}+\sigma(\ell), b_{z}>b_{x_{j}}-\sigma(\ell), a_{z}<a_{\mathcal{R} x_{j}}+\sigma(\ell), b_{z}>b_{\mathcal{R} x_{j}}-\sigma(\ell) \forall j=1, \ldots, k \\
\text { and } \tau \in\left[0, \tau_{3}(\ell)\right] \Longrightarrow \frac{\partial \Delta_{z}}{\partial \tau}(\tau) \leq\left(b_{\tau}-a_{\tau}\right)\left(-\frac{\mu(\ell)}{4}\right) \leq-\frac{c_{1} \mu(\ell)}{8 M_{0}},
\end{gathered}
$$

because $b_{\tau}-a_{\tau} \geq \frac{c_{1}}{2 M_{0}}$.

Note that if there exists $i$ such that

$a_{z} \geq a_{x_{i}}+\sigma(\ell)$ or $b_{z} \leq b_{x_{i}}-\sigma(\ell)$, and

$$
a_{z} \geq a_{\mathcal{R} x_{i}}+\sigma(\ell) \text { or } b_{z} \leq b_{\mathcal{R} x_{i}}-\sigma(\ell)
$$

and $\rho_{1}\left(x_{i}\right)$ is chosen sufficiently small we have

(10.21) $z \in \mathcal{U}_{x_{i}} \Longrightarrow\left(b_{z}-a_{z}\right) \frac{1}{2} \int_{a_{z}}^{b_{z}} g(\dot{z}, \dot{z}) \mathrm{d} s \leq\left(b_{x_{i}}-a_{x_{i}}\right) \frac{1}{2} \int_{a_{x_{i}}}^{b_{x_{i}}} g(\dot{x}, \dot{x}) \mathrm{d} s-\Delta(\ell)$,

where $\Delta(\ell)$ is a positive constant depending only by $\ell$, as we can see arguing as in the proof of Lemma 9.2. Therefore, arguing as in the proof of Lemma 9.5, we see that $\Delta_{z}(\tau)$ is always under the level $c_{1}-\epsilon$ for any $\tau \in\left[0, \tau_{3}(\ell)\right]$, for a suitable choice of $\left.\left.\tau_{3}(\ell) \in\right] 0, \tau_{2}(\ell)\right]$.

To conclude the proof we can therefore repeat the proof of Proposition 9.1 outside a small neighborhood of irregular variationally critical portions of II type, using the flow (10.17) is such a neighborhood. Using essentially the same approach of the proof of Proposition 9.1 we can arrive to the conclusion thanks in particular to properties (10.19), (10.20) and (10.21).

Remark 10.10. In the proof above we use $\sigma(\ell)$ (involving lengths of intervals) instead of $E_{1}(\ell)$ because it may happens that $D\left(x_{i}, a_{z}, b_{z}, a_{x_{i}}, b_{x_{i}}\right)$ is small and the differences $a_{z}-a_{x_{i}}$ or $b_{x_{i}}-b_{z}$ are positive and big, since we are closed to curve that may be constant in such intervals. Note also that here $b_{\tau}-a_{\tau}$ may be increasing, differently from the situation of section 9 where $b_{\tau}-a_{\tau}$ is always not increasing. 


\section{A DEFORMATION RESUlT FOR HOMOTOPIES OF TYPE C AND THE FIRST DEFORMATION LEMMA}

In this section we shall give statement and proof of the analogous of the classical First Deformation Lemma for the functional $\mathcal{F}$. This is the key to obtain the existence result.

Before to do this we give a deformation result involving homotopies of type $\mathrm{C}$ used to move far from irregular variationally critical pieces of I type.

Let $\rho_{0}$ be given by Proposition 7.6.

Proposition 11.1. There exists $\bar{r}>0$ with the following property:

for all $(h, \mathcal{D}) \in \mathcal{H}_{1}$ there exists a continuous homotopy $H_{0}:[0,1] \times h(1, \mathcal{D}) \rightarrow \mathfrak{M}$ of type $C$ such that

(1) $\left(\mathcal{D}, H_{0} \star h\right) \in \mathcal{H}_{1}$;

(2) $\mathcal{F}\left(\mathcal{D}, H_{0} \star h\right) \leq \mathcal{F}(\mathcal{D}, h)$;

(3) $\left\|H_{0}(\tau, x)-x\right\|_{0,1} \leq \tau \frac{\rho_{0}}{2}$, for all $x \in h(1, \mathcal{D})$, for all $\tau \in[0,1]$;

(4) for every $x \in h(1, \mathcal{D})$, and for every $[a, b] \in \mathcal{I}_{x}$ it is

$$
\left\|H_{0}(1, x)-y\right\|_{\alpha, \beta} \geq \bar{r}
$$

for any $y \in \mathfrak{M}$, and for any $[\alpha, \beta] \subset[a, b]$ topologically not essential interval for $y$.

Proof. The proof is divided into two parts: first part requires the construction of the vector field (with the help of Proposition 7.6) generating the flow that we shall study in the second part of the proof.

\section{Part A: Construction of the vector field.}

Set

$$
\begin{aligned}
X=\left\{x \in h(1, \mathcal{D}): \exists[\alpha, \beta] \subset[a, b] \in \mathcal{I}_{x}, \bar{\delta} \text {-close to } \partial \Omega\right. \\
\left.\quad \text { and such that } \mathfrak{p}_{\alpha, \beta}^{x} \in\left[-2 \sigma_{1},-\frac{\sigma_{1}}{3}\right], \mathfrak{b}_{\alpha, \beta}^{x} \geq 1+\bar{\gamma}\right\},
\end{aligned}
$$

where $\bar{\delta}$ is given by $(7.8)$ and $\sigma_{1}$ by Remark 7.7. Note that $X$ is a compact set. For any $x \in X$ denote by $\left[\alpha_{x}^{i}, \beta_{x}^{\imath}\right], i=1, \ldots, k(x)$ the intervals $[\alpha, \beta]$ described in the definition above. Note that, by Lemma 2.1, their number, $k(x)$ is bounded by a constant independent of $x, \mathcal{D}$ and $h$. Moreover due to their minimality property, for any $i \neq j,\left[\alpha_{x}^{i}, \beta_{x}^{i}\right] \cap\left[\alpha_{x}^{j}, \beta_{x}^{j}\right]$ has at most one element. Denote by $\left[\tilde{\alpha}_{x}, \tilde{\beta}_{x}\right]$ the summary interval for $x$ (cf. Definition 7.5) including the intervals $\left[\alpha_{x}^{i}, \beta_{x}^{i}\right]$.

Let $\rho_{0}, \theta_{0}, \varepsilon_{0}$ and $\mu_{0}$ be as in Proposition 7.6. Thanks to it, for any $x \in X$ there exist $\left.\rho(x) \in] 0, \frac{\rho_{0}}{2}\right]$ and $V_{x} \in H_{0}^{1}\left([0,1], \mathbb{R}^{N}\right)\left(\right.$ extended as null vector field outside $\left.\left[\tilde{\alpha}_{x}, \tilde{\beta}_{x}\right]\right)$ such that:

- $V_{\mathcal{R} x}(s)=V_{x}(1-s)=\mathcal{R} V_{x}(s)$ for all $s \in[0,1]$,

- $\left\|V_{x}\right\|_{0,1}=1$,

- $V_{x}(s)=0$, for any $s$ such that $\phi\left(x(s) \leq-\bar{\delta}+\varepsilon_{0}\right.$,

and for any $z$ satisfying $\|z-x\|_{0,1}<\rho(x)$ it is:

- $\phi(z(s)) \leq-\frac{\sigma_{1}}{4}$ for all $s \in\left[\tilde{\alpha}_{x}, \tilde{\beta}_{x}\right]$,

- $V_{x}(s)=0$, for any $s$ such that $\phi\left(z(s) \leq-\bar{\delta}+\varepsilon_{0}\right.$,

- $g\left(\nabla \phi(z(s)), V_{x}(s)\right) \leq-\theta_{0}$ for all $s \in\left[\tilde{\alpha}_{x}, \tilde{\beta}_{x}\right]$ such that $\phi(z(s)) \in\left[-2 \sigma_{0}, 0\right]$,

- $\int_{\tilde{\alpha}_{x}^{i}}^{\tilde{\beta}^{i}} g\left(\dot{z}, \frac{\mathrm{D}}{\mathrm{d} s} V_{x}\right) \mathrm{d} s \leq-\mu_{0}$.

Now set

$$
\mathcal{U}_{x}=\left\{z \in \mathfrak{M}:\|z-x\|_{0,1}<\rho(x)\right\}
$$


and consider the open covering $\left\{\mathcal{U}_{x}, \mathcal{U}_{\mathcal{R} x}\right\}_{x \in X}$ of $X$. Since $X$ is compact there exists a finite covering $\left\{\mathcal{U}_{x_{i}}, \mathcal{U}_{\mathcal{R} x_{i}}\right\}_{i=1, \ldots, k}$ of $X$. Set

$$
\mathcal{U}_{X}=\bigcup_{i=1, \ldots, k}\left(\mathcal{U}_{x_{i}} \cup \mathcal{U}_{\mathcal{R} x_{i}}\right)
$$

Define, for any $x \in \overline{\mathcal{U}_{x}}$,

$$
\varrho_{x_{i}}(z)=\operatorname{dist}_{0,1}\left(z, \overline{\mathcal{U}_{X}} \backslash \mathcal{U}_{x_{i}}\right)
$$

where dist ${ }_{0,1}$ is the distance induced by the norm $\|\cdot\|_{0,1}$. Since $\mathcal{R} X=X, \mathcal{R} \mathcal{U}_{x_{i}}=\mathcal{U}_{\mathcal{R} x_{i}}$ and $\mathcal{R} \circ \mathcal{R}$ is the identity map, it is

$$
\varrho_{x_{i}}(\mathcal{R} z)=\varrho_{\mathcal{R} x_{i}}(z) .
$$

Finally set

which satisfies

$$
\beta_{x_{i}}(z)=\frac{\varrho_{x_{i}}(z)}{\sum_{j=1, \ldots, k}\left(\varrho_{x_{j}}(z)+\varrho_{\mathcal{R} x_{j}}(z)\right)},
$$

and

$$
\beta_{x_{i}}(\mathcal{R} z)=\beta_{\mathcal{R} x_{i}}(z)
$$

$$
\sum_{j=1, \ldots, k}\left(\beta_{x_{j}}(z)+\beta_{\mathcal{R} x_{j}}(z)\right)=1 .
$$

The vector field that we shall use in this proof is defined as

$$
W(z)(s)=\sum_{j=1, \ldots, k}\left(\beta_{x_{j}}(z) V_{x_{j}}(s)+\beta_{\mathcal{R} x_{j}}(z) V_{\mathcal{R} x_{j}}(s)\right),
$$

which is well defined on all $\mathfrak{M}$.

Note that by the definition of $V_{x}$

$$
\|W(z)\|_{0,1} \leq 1 .
$$

Moreover, by the $\mathcal{R}$-equivariant choice of $V_{x_{i}}$ and the $\mathcal{R}$-invariant property of $\beta_{x_{i}}$ it is

$$
\mathcal{R} W(z)=W(\mathcal{R} z) .
$$

The existence of the homotopy $H_{0}$ will be proved studying the flow $\eta(\tau, z)$ given by the solution of the initial value problem

$$
\left\{\begin{array}{l}
\frac{\mathrm{d}}{\mathrm{d} \tau} \eta(s)=W(z)(s) \\
\eta(0)=z \in \overline{\mathcal{U}_{X}}
\end{array}\right.
$$

whose solution (for any $\tau \geq 0$ ) is given by

$$
\eta(\tau, z)(s)=z(s)+\tau W(z)(s) .
$$

Part B: Properties of the flow $\eta$.

We shall move by $\eta$ only the open set $\mathcal{U}_{X}$. More precisely let $\mathcal{V}_{X}$ an $\mathcal{R}$-equivariant open neighborhood of $X$ such that

$$
X \subset \mathcal{V}_{X} \subset \overline{\mathcal{V}_{X}} \subset \mathcal{U}_{X},
$$

and consider a $\mathcal{R}$-invariant continuous map $\chi: \mathfrak{M} \rightarrow[0,1]$ such that

$$
\chi(z)=1 \text { if } z \in \overline{\mathcal{V}_{X}}, \chi(z)=0 \text { if } z \in \mathfrak{M} \backslash \mathcal{U}_{X} .
$$

We shall consider

$$
H(\tau, z)=\eta(\chi(z) \tau, z) .
$$

Arguing as in proving Step 1 of Proposition 9.1 we see that $H$ is $\mathcal{R}$-equivariant. Moreover choosing $\rho(x)$ sufficiently small $\mathcal{U}_{X}$ is far from the constat curves, so $H$ does not move them. 
Since $\rho\left(x_{i}\right) \leq \frac{\rho_{0}}{2}$, arguing as in step 5 of Proposition 9.1, se see that

if $\tau_{*}$ is the first instant such that $\left\|\eta(\tau, z)-x_{i}\right\|_{0,1}=\rho_{0}$

$$
\text { for some } i \text { and } z \in \mathcal{U}_{x_{i}} \text {, then } \tau_{*} \geq \frac{\rho_{0}}{2} \text {. }
$$

Moreover, any $V_{x_{i}}$ satisfies

$$
g\left(\nabla \phi(z(s)), V_{x_{i}}(s)\right) \leq-\theta_{0} \text { for all } s \in\left[\tilde{\alpha}_{x}, \tilde{\beta}_{x}\right] \text { such that } \phi(z(s)) \in\left[-2 \sigma_{0}, 0\right],
$$

while $V_{x_{i}}=0$ outside the intervals $\left[\tilde{\alpha}_{x}, \tilde{\beta}_{x}\right]$. Then, thanks to condition (8.5) satisfied by $h$, if we choose $\rho(x)$ sufficiently small we see that $H$ is of type $\mathrm{C}$ in $\left[0, \frac{\rho_{0}}{2}\right]$ (and that any $\left[a_{z}, b_{z}\right] \in \mathcal{I}_{z}$ does not change along the flow).

Note also that, due to the properties of the $V_{x_{i}}$ 's we have

$$
\frac{1}{2} \int_{a_{z}}^{b_{z}} g\left(\dot{z}_{\tau}, \dot{z}_{\tau}\right) \mathrm{d} s \leq \frac{1}{2} \int_{a_{z}}^{b_{z}} g(\dot{z}, \dot{z}) \mathrm{d} s
$$

where $z_{\tau}=\eta(\tau, z)$, for any $\tau \in\left[0, \frac{\rho_{0}}{2}\right]$, for any $z \in \mathfrak{M}$.

Then, setting

$$
H_{0}(\tau, z)=H\left(\tau \frac{\rho_{0}}{2}, z\right),
$$

we see that $\mathcal{F}\left(\mathcal{D}, h \star H_{0}\right) \leq \mathcal{F}(\mathcal{D}, h)$, while by 11.2

$$
\left\|H_{0}(\tau, x)-x\right\|_{0,1} \leq \tau \frac{\rho_{0}}{2} \text { for any } x \in h(1, \mathcal{D}) .
$$

To conclude also that $\left(\mathcal{D}, h \star H_{0}\right) \in \mathcal{H}_{1}$, it is to observe that if the radius of any $\mathcal{U}_{x_{i}}$ is sufficiently small the endpoints of the restriction of the curve $z$ to a topologically not essential interval are not moved by the flow, which implies that the bending constant is not affected by the flow. Moreover the flow do not creates news topologically non essential intervals since nothing moves nearby the level $-\bar{\delta}$. Then, along the flow, intervals which are far to be topologically not essential still remain far to be topologically not essential.

Finally by the choice of any $V_{x_{i}}$ we see also that, starting from $z \in \overline{\mathcal{V}_{X}}$, the map $\phi$ decreases along the flow $\eta$ with derivative $\leq-\theta_{0}$ until the maximal proximity to $\partial \Omega$ gets to the value $-2 \sigma_{1}$ and we have, for any $\tau \in\left[0, \frac{\rho_{0}}{2}\right]$ and $z \in \overline{\mathcal{V}_{X}}$ such that $\phi(\eta(\tau, z)) \geq-2 \sigma_{1}$ :

$$
\phi(\eta(\tau, z)) \leq \phi(\eta(0, z))-\tau \theta_{0} \leq-\frac{\sigma_{1}}{4}-\tau \theta_{0},
$$

while $\sigma_{1}$ has been chosen so that $\sigma_{1} \leq \frac{2}{7} \rho_{0} \theta_{0}$ (cf. Remark 7.7). Then $\phi(\eta(\cdot, z))$ reaches surely the level $-2 \sigma_{1}$ in a time $\tau \in\left[0, \frac{\rho_{0}}{2}\right]$ and we can finally deduce the existence of $\bar{r}>0$ satisfying (4).

We are now ready for the following:

Proposition 11.2 (First Deformation Lemma). Let $c \geq \frac{1}{2}\left(\frac{3 \delta_{0}}{4 K_{0}}\right)^{2}$ be a geometrically regular value (cf. Definition 3.1). Then it is a topologically regular value of $\mathcal{F}$, namely there exists $\varepsilon=\varepsilon(c)>0$ having the following property: for all $(\mathcal{D}, h) \in \mathcal{H}_{1}$ with

$$
\mathcal{F}(\mathcal{D}, h) \leq c+\varepsilon
$$

there exists a continuous map $\eta \in C^{0}([0,1] \times h(1, \mathcal{D}), \mathfrak{M})$ such that $(\mathcal{D}, \eta \star h) \in \mathcal{H}_{1}$ and

$$
\mathcal{F}(\mathcal{D}, \eta \star h) \leq c-\varepsilon .
$$

Proof. Take $\bar{\varepsilon}>0$ such that there are no OGC's in $\bar{\Omega}$ with energy value in $[c-\bar{\varepsilon}, c+\bar{\varepsilon}]$. A simple contradiction argument shows the existence of $r_{c}>0$ such that the conditions $x \in \mathfrak{M},[a, b] \in \mathcal{I}_{x}$, and $\frac{(b-a)}{2} \int_{a}^{b} g(\dot{x}, \dot{x}) \mathrm{d} s \in[c-\bar{\varepsilon}, c+\bar{\varepsilon}]$ imply $\|x-y\|_{a, b} \geq r_{c}$ for all $y:[a, b] \rightarrow \bar{\Omega}$ affinely parameterized OGC in $\bar{\Omega}$.

Using the homotopy $H_{0}$ of Proposition 11.1 we can move $h(1, \mathcal{D})$ in such a way that

$$
\mathcal{F}\left(\mathcal{D}, H_{0} \star h\right) \leq \mathcal{F}(\mathcal{D}, h)
$$


and it becomes far from the set of paths having topologically not essential intervals.

Therefore, choosing $\varepsilon>0$ sufficiently small and applying Proposition 10.3, we obtain the existence of a homotopy $H_{\varepsilon}$ such that $\left(\mathcal{D}, H_{\varepsilon} \star H_{0} \star h\right) \in \mathcal{H}_{1}$ and

$$
\mathcal{F}\left(\mathcal{D}, H_{\varepsilon} \star H_{0} \star h\right) \leq \mathcal{F}\left(\mathcal{D}, H_{0} \star h\right)-2 \varepsilon \leq \mathcal{F}(\mathcal{D}, h)-2 \varepsilon
$$

which implies

$$
\mathcal{F}\left(\mathcal{D}, H_{\varepsilon} \star H_{0} \star h\right) \leq c-\varepsilon .
$$

Then $H_{\varepsilon} \star H_{0} \star h$ is the required homotopy.

\section{Proof of the main Theorem}

In view of multiplicity results the topological invariant that will be employed in our proof is the relative category of a topological pair $(X, Y)$ as defined in [12, Definition 3.1]. For other definition of the relative category and other relative cohomological indexes see e.g. [11] and references therein.

Definition 12.1. Let $X$ be a topological space and $Y$ a closed subset of $X$. A closed subset $F$ of $X$ has relative category equal to $k \in \mathbb{N}\left(\operatorname{cat}_{X, Y}(F)=k\right.$ ) if $k$ is the minimal positive integer such that $F \subset \cup_{i=0}^{k} A_{i}$, any $A_{i}$ is open, $F \cap Y \subset A_{0}$, and for any $i=0, \ldots, k$ there exists $h_{i} \in C^{0}\left([0,1] \times A_{i}, X\right)$ with the following properties:

(1) $h_{1}(0, x)=x, \forall x \in A_{i}, \forall i=0, \ldots, k$;

(2) for any $i=1, \ldots, k$ :

(a) there exists $x_{i} \in X \backslash Y$ such that $h_{i}\left(1, A_{i}\right)=\left\{x_{i}\right\}$;

(b) $h_{i}:\left([0,1] \times A_{i}\right) \subset X \backslash Y$;

(3) if $i=0$ :

(a) $h_{0}\left(1, A_{0}\right) \subset Y$;

(b) $h_{0}\left(\tau, A_{0} \cap Y\right) \subset Y, \forall \tau \in[0,1]$.

For any $X \in \mathfrak{M}$, we denote by $\widetilde{X}$ the quotient space with respect to the equivalence relation induced by $\mathcal{R}$. We shall use, as topological invariant, the number cat $\widetilde{\mathfrak{C}}_{\tilde{\mathfrak{c}}} \widetilde{\mathfrak{c}}_{0}(\widetilde{\mathfrak{C}})$. In Appendix $A$ we will show that

$$
\operatorname{cat}_{\widetilde{\mathfrak{C}}, \widetilde{\mathfrak{C}_{0}}}(\widetilde{\mathfrak{C}}) \geq N
$$

using the topological properties of the $(N-1)$-dimensional real projective space.

Denote by $\mathfrak{D}$ the class of closed $\mathcal{R}$-invariant subset of $\mathfrak{C}$. Define, for any $i=1, \ldots, N$,

$$
\Gamma_{i}=\left\{\mathcal{D} \in \mathfrak{D}: \operatorname{cat}_{\widetilde{\mathfrak{C}}, \widetilde{\mathfrak{C}}_{0}}(\widetilde{\mathcal{D}}) \geq i\right\}
$$

Set

$$
c_{i}=\inf _{\substack{\mathcal{D} \in \Gamma_{i},(\mathcal{D}, h) \in \mathcal{H}_{1}}} \mathcal{F}(\mathcal{D}, h) .
$$

Any number $c_{i}$ is called topologically critical level or $\mathcal{F}$.

Remark 12.2. If $\mathrm{I}_{\mathfrak{C}}:[0,1] \times \mathfrak{C}$ denotes the map $\mathrm{I}_{\mathfrak{C}}(\tau, x)=x$ for all $\tau$ and all $x$, the the pair $\left(\mathfrak{C}, I_{\mathfrak{C}}\right) \in \mathcal{H}_{1}$. Since $\widetilde{\mathfrak{C}} \in \Gamma_{i}$ for any $i($ see $(12.1)$ ), we get:

$$
c_{i} \leq \mathcal{F}\left(\mathfrak{C}, \mathrm{I}_{\mathfrak{C}}\right)<M_{0}
$$

Moreover $\mathcal{F} \geq 0$, therefore $0 \leq c_{i} \leq M_{0}$ for any $i$ (recall also the definition of $\mathcal{F}$ and $\left.M_{0}\right)$.

We have the following lemmas involving the real numbers $c_{i}$.

Lemma 12.3. The following statements hold:

(1) $c_{1} \geq \frac{1}{2}\left(\frac{3 \delta_{0}}{4 K_{0}}\right)^{2}$; 
(2) $c_{1} \leq c_{2} \leq \cdots \leq c_{N}$.

Lemma 12.4. For all $i=1, \ldots, N, c_{i}$ is a geometrically critical value.

Proof of Lemma 12.3. Let us prove (1). Assume by contradiction $c_{1}<\frac{1}{2}\left(\frac{3 \delta_{0}}{4 K_{0}}\right)^{2}$, and take $\varepsilon>0$ such that $c_{1}+\varepsilon<\frac{1}{2}\left(\frac{3 \delta_{0}}{4 K_{0}}\right)^{2}$. By 12.2 12.3) there exists $\mathcal{D}_{\varepsilon} \in \Gamma_{1}$, and $\left(\mathcal{D}_{\varepsilon}, h_{\varepsilon}\right) \in \mathcal{H}_{1}$ such that

$$
\mathcal{F}\left(\mathcal{D}_{\varepsilon}, h_{\varepsilon}\right) \leq c_{1}+\varepsilon<\frac{1}{2}\left(\frac{3 \delta_{0}}{4 K_{0}}\right)^{2} .
$$

Then there exists an $\mathcal{R}$-equivariant homotopy $h_{0}$ sending the curve $h_{\varepsilon}\left(1, \mathcal{D}_{\varepsilon}\right)$ first on $\partial \Omega$ (sending constant curves in constant curves) and then on the constant curves of $\partial \Omega$, moving the extreme points along the curves themselves. So $\left(h_{0} \star h_{\varepsilon}\right)\left(1, \mathcal{D}_{\varepsilon}\right)$ consists of constant curves in $\partial \Omega$ (and $h_{0} \star h_{\varepsilon}$ sends the constant curves of $\mathcal{D}_{\varepsilon}$ in constant curves). Then there exist a homotopy $K_{\varepsilon}:[0,1] \times \widetilde{\mathcal{D}}_{\varepsilon} \rightarrow \widetilde{\mathfrak{C}}$ such that $K_{\varepsilon}(0, \cdot)$ is the identity, $K_{\varepsilon}\left(1, \widetilde{\mathcal{D}}_{\varepsilon}\right) \subset \widetilde{\mathfrak{C}}_{0}$ and

$$
K_{\varepsilon}\left(\tau, \widetilde{\mathcal{D}}_{\varepsilon} \cap \widetilde{\mathfrak{C}}_{0}\right) \subset \widetilde{\mathfrak{C}}_{0}, \forall \tau \in[0,1] .
$$

Then $\operatorname{cat}_{\widetilde{\mathfrak{C}}, \widetilde{\mathfrak{C}}_{0}}\left(\widetilde{\mathcal{D}}_{\varepsilon}\right)=0$, in contradiction with the definition of $\Gamma_{1}$.

To prove (2), fix $i \in\{1, \ldots, N-1\}$ and consider $c_{i}$ and $c_{i+1}$. By (12.3) for any $\varepsilon>0$ there exists $\mathcal{D} \in \Gamma_{i+1}$ and $(\mathcal{D}, h) \in \mathcal{H}_{1}$ such that

$$
\mathcal{F}(\mathcal{D}, h) \leq c_{i+1}+\varepsilon
$$

Since $\Gamma_{i+1} \subset \Gamma_{i}$ by definition of $c_{i}$ we deduce $c_{i} \leq c_{i+1}+\varepsilon$, and (2) is proved, since $\varepsilon$ is arbitrary.

Proof of Lemma 12.4. Assume by contradiction that $c_{i}$ is not a geometrically critical value for some $i$. Take $\varepsilon=\varepsilon\left(c_{i}\right)$ as in Proposition 11.2, and $\left(\mathcal{D}_{\varepsilon}, h\right) \in \mathcal{H}_{1}$ such that

$$
\mathcal{F}\left(\mathcal{D}_{\varepsilon}, h\right) \leq c_{i}+\varepsilon .
$$

Now let $\eta$ as in Proposition 11.2 and take $h_{\varepsilon}=\eta \star h$. Since

$$
\mathcal{F}\left(\mathcal{D}_{\varepsilon}, h_{\varepsilon}\right) \leq c_{i}-\varepsilon,
$$

we get a contradiction with $(12.3)$ because $\left(\mathcal{D}_{\varepsilon}, h_{\varepsilon}\right) \in \mathcal{H}_{1}$.

Proof of Theorem. It follows from part (11) of Lemma 12.3 and Lemma 12.4 .

Remark 12.5. Note that we have obtained only an existence result; a multiplicity result would follow from our construction if one provided additional arguments that show that the $c_{i}$ 's are distinct. This could be done if one were able to determine open contractible sets of our path space, containing curves having portions close to OGC's. The details of this construction are rather involved, and they are the object of further studies.

\section{APPENDiX A. COMPUTATION OF THE RELATIVE CATEGORY}

Let $2 \leq N \in \mathbb{N}, \mathbb{D}^{N}$ the unit disk in $\mathbb{R}^{N}$ with the Euclidean norm and $\mathbb{S}^{N-1}=\partial \mathbb{D}^{N}$. Let $\mathcal{R}$ be the reversing map $\mathcal{R} x(s)=x(1-s)$, defined in the set of curves $x:[0,1] \rightarrow \mathbb{D}^{N}$. With a slight abuse of notation we will denote by $\mathcal{R}$ also the equivalence relation induced. Fix $\sigma \in] 0,1[$ and set

$$
\begin{aligned}
& \mathcal{C}=\left\{\gamma:[0,1] \rightarrow \mathbb{D}^{N}, \gamma(t)=(1-t) x_{1}+t x_{2}, x_{1}, x_{2} \in \mathbb{S}^{N-1}\right\} / \mathcal{R}, \\
& \mathcal{C}_{\sigma}=\{[\gamma] \in \mathcal{C}:|\gamma(1)-\gamma(0)| \leq \sigma\},
\end{aligned}
$$

and

$$
\mathcal{C}_{0}=\left\{[\gamma] \in \mathcal{C}: \gamma(t)=x \in \mathbb{S}^{N-1} \forall t \in[0,1]\right\}
$$


Note that $\mathcal{C}$ is homeomorphic to $\left(\mathbb{S}^{N-1} \times \mathbb{S}^{N-1}\right) / \mathbb{Z}_{2}$ where the action of $\mathbb{Z}_{2}$ on $\mathbb{S}^{N-1} \times$ $\mathbb{S}^{N-1}$ is given by $\mathcal{S}(A, B)=(B, A)$.

Remark A.1. Since there exists an homeomorphism $\Phi: \mathcal{C} \rightarrow \mathfrak{C}$ such that $\Phi\left(\mathcal{C}_{0}\right)=\mathfrak{C}_{0}$, then

$$
\operatorname{cat}_{\mathcal{C}, \mathcal{C}_{0}}(\mathcal{C})=\operatorname{cat}_{\widetilde{\mathfrak{C}}, \widetilde{\mathfrak{C}_{0}}}(\widetilde{\mathfrak{C}})
$$

therefore, to prove (12.1), we need to show that $\operatorname{cat}_{\mathcal{C}, \mathcal{C}_{0}}(\mathcal{C}) \geq N$.

Remark A.2. Note that property (2b) of Definition 12.1 is essential in our case, to guarantee that the relative category is at least $N$. Indeed in $[$ it is proved that the LjusternikSchnirelman category of $\mathcal{C}$ is at most equal to three, and if we did not require (2b) in the definition of relative category we would have $\operatorname{cat}_{\mathcal{C}, \mathcal{C}_{0}}(\mathcal{C}) \leq \operatorname{cat}(\mathcal{C})$.

Now we are going to prove the following result.

Proposition A.3. $\operatorname{cat}_{\mathfrak{C}, \mathfrak{c}_{0}}(\mathfrak{C}) \geq N$.

The proof will be performed using singular cohomology theory and the cup product (see e.g. [18]) with $\mathbb{Z}_{2}$ coefficients. For any topological pair $(X, Y)$ it will be denoted by $H^{q}(X, Y)$ at any dimension $q \geq 0$.

The notion of relative cuplength, here recalled, will be also used.

Definition A.4. The number cuplenght $(X, Y)$ is the largest positive integer $k$ for which there exists $\alpha_{0} \in H^{q_{0}}(X, Y)\left(q_{0} \geq 0\right)$ and $\alpha_{i} \in H^{q_{i}}(X), i=1, \ldots, k$ such that

$$
q_{i} \geq 1 \forall i=1, \ldots, k
$$

and

$$
\alpha_{0} \cup \alpha_{1} \cup \ldots \cup \alpha_{k} \neq 0 \text { in } H^{q_{0}+q_{1}+\ldots+q_{k}}(X, Y),
$$

where $\cup$ denotes the cup product.

Recall that, if $Y \neq \emptyset$, the absolute cuplenght of $X$ is the largest positive integer $k$ for which there exists $\alpha_{i} \in H^{q_{i}}(X), i=1, \ldots, k$ such that

$$
q_{i} \geq 1, \forall i=1, \ldots, k,
$$

and

$$
\alpha_{1} \cup \ldots \alpha_{k} \neq 0 \text { in } H^{q_{1}+\ldots+q_{k}}(X) .
$$

Proof of Proposition A.3. The proof is divided into four steps.

Step 1. $\operatorname{cat}_{\mathcal{C}, \mathcal{C}_{0}}(\mathcal{C}) \geq \operatorname{cuplenght}\left(\mathcal{C} \backslash \mathcal{C}_{0}, \mathcal{C}_{\sigma} \backslash \mathcal{C}_{0}\right)+1$.

Assume that cat ${ }_{\mathcal{C}, \mathcal{C}_{0}}(\mathcal{C})=k<+\infty$. Since $\mathcal{C}_{0}$ is not a retract of $\mathcal{C}$, it is $k \geq 1$. Take $A_{0}, A_{1}, \ldots, A_{k}$ open subsets as in definition 12.1, and let

$$
\imath_{r}: A_{r} \rightarrow \mathcal{C} \backslash \mathcal{C}_{0}, \quad \jmath_{r}:\left(\mathcal{C} \backslash \mathcal{C}_{0}, \emptyset\right) \rightarrow\left(\mathcal{C} \backslash \mathcal{C}_{0}, A_{r}\right)
$$

be inclusion maps. By property (2) of Definition 12.1, $\imath_{r}^{*}: H^{q}\left(\mathcal{C} \backslash \mathcal{C}_{0}\right) \rightarrow H^{q}\left(A_{r}\right)$ is the zero constant map for any $q \geq 1$ and any $r \geq 1$. Then, since the sequence

$$
\ldots \rightarrow H^{q_{r}}\left(\mathcal{C} \backslash \mathcal{C}_{0}, A_{r}\right) \stackrel{\jmath_{r}^{*}}{\longrightarrow} H^{q_{r}}\left(\mathcal{C} \backslash \mathcal{C}_{0}\right) \stackrel{\imath_{r}^{*}}{\rightarrow} H^{q_{r}}\left(A_{r}\right) \rightarrow \ldots
$$

is exact, then $\jmath_{r}^{*}$ is surjective if $q_{r} \geq 1$. Then for any $\alpha_{r} \in H^{q_{r}}\left(\mathcal{C} \backslash \mathcal{C}_{0}\right)$, if $q_{r} \geq 1$, there exists $\beta_{r} \in H^{q_{r}}\left(\mathcal{C} \backslash \mathcal{C}_{0}, A_{r}\right)$ such that $\jmath_{r}^{*}\left(\beta_{r}\right)=\alpha_{r}$.

Since $A_{0} \supset \mathcal{C}_{0}, A_{0}$ is open and $\mathcal{C}_{0}$ is closed, there exists $\left.\sigma \in\right] 0,1\left[\right.$ such that $A_{0} \supset \mathcal{C}_{\sigma}$. Moreover by property (3b) of Definition 12.1, $\sigma$ can be chosen sufficiently small so that, up to consider a projection on $\mathcal{C}_{0}$, a homotopy $\hat{h}_{0}$ can be built such that $\hat{h}_{0}\left(\tau, \mathcal{C}_{\sigma}\right) \subset \mathcal{C}_{\sigma}, \forall \tau \in$ $[0,1]$, (while, obviously, $\left.\hat{h}_{0}\left(1, A_{0}\right) \subset \mathcal{C}_{\sigma}\right)$.

Now consider the inclusion maps

$$
\jmath_{0}:\left(\mathcal{C}, \mathcal{C}_{\sigma}\right) \rightarrow\left(\mathcal{C}, A_{0}\right), \quad \iota_{0}:\left(A_{0}, \mathcal{C}_{\sigma}\right) \rightarrow\left(\mathcal{C}, \mathcal{C}_{\sigma}\right),
$$


and the (exact) sequence

$$
\ldots \rightarrow H^{q_{0}}\left(\mathcal{C}, A_{0}\right) \stackrel{\jmath_{0}^{*}}{\longrightarrow} H^{q_{0}}\left(\mathcal{C}, \mathcal{C}_{\sigma}\right) \stackrel{\imath_{0}^{*}}{\longrightarrow} H^{q_{0}}\left(A_{0}, \mathcal{C}_{\sigma}\right) \rightarrow \ldots
$$

Since $\imath_{0}^{*}: H^{q_{0}}\left(\mathcal{C}, \mathcal{C}_{\sigma}\right) \rightarrow H^{q_{0}}\left(A_{0}, \mathcal{C}_{\sigma}\right)$ is the constant zero map then $j_{0}^{*}$ is surjective and, for any $\alpha_{0} \in H^{q_{0}}\left(\mathcal{C}, \mathcal{C}_{\sigma}\right)$ there exists $\beta_{0} \in H^{q_{0}}\left(\mathcal{C}, A_{0}\right)$ such that $g_{0}^{*}\left(\beta_{0}\right)=\alpha_{0}$. Now $\mathcal{C}_{0}$ is a strong deformation retract of $\mathcal{C}_{\sigma}$ since $\sigma<1$, and by excision property (recalling that $\mathcal{C}_{\sigma} \subset$ $\left.A_{0}\right)$ we have that for any $\hat{\alpha}_{0} \in H^{q_{0}}\left(\mathcal{C} \backslash \mathcal{C}_{0}, \mathcal{C}_{\sigma} \backslash \mathcal{C}_{0}\right)$ there exists $\hat{\beta}_{0} \in H^{q_{0}}\left(\mathcal{C} \backslash \mathcal{C}_{0}, A_{0} \backslash \mathcal{C}_{0}\right)$ such that

$$
\jmath_{0}^{*}\left(\hat{\beta}_{0}\right)=\hat{\alpha}_{0},
$$

where $\jmath_{0}:\left(\mathcal{C} \backslash \mathcal{C}_{0}, \mathcal{C}_{\sigma} \backslash \mathcal{C}_{0}\right) \rightarrow\left(\mathcal{C} \backslash \mathcal{C}_{0}, A_{0} \backslash \mathcal{C}_{0}\right)$ is the inclusion map.

Finally we have, since $A_{i}$ are open,

$$
\begin{aligned}
\hat{\beta}_{0} \cup \beta_{1} \cup \ldots \cup \beta_{k} \in H^{q_{0}+q_{1}+\ldots+q_{k}}\left(\mathcal{C} \backslash \mathcal{C}_{0},\left(A_{0} \backslash \mathcal{C}_{0}\right) \cup A_{1} \cup \ldots \cup A_{k}\right)= \\
H^{q_{o}+q_{1}+\ldots+q_{k}}\left(\mathcal{C} \backslash \mathcal{C}_{0}, \mathcal{C} \backslash \mathcal{C}_{0}\right)=0 .
\end{aligned}
$$

Moreover, by the naturality of the cup product (see [18]) we have (denoting by $\jmath$ the inclusion map)

$$
\hat{\alpha}_{0} \cup \alpha_{1} \cup \ldots \cup \alpha_{k}=\jmath^{*}\left(\hat{\beta}_{0} \cup \beta_{1} \cup \ldots \cup \beta_{k}\right)=\jmath^{*}(0)=0,
$$

proving that cuplenght $\left(\mathcal{C} \backslash \mathcal{C}_{0}, \mathcal{C}_{\sigma} \backslash \mathcal{C}_{0}\right)<k$.

Step 2. cuplenght $\left(\mathcal{C} \backslash \mathcal{C}_{0}, \mathcal{C}_{\sigma} \backslash \mathcal{C}_{0}\right)=\operatorname{cuplenght}\left(X_{\sigma}, Y_{\sigma}\right)$, where

$$
X_{\sigma}=\{[\gamma] \in \mathcal{C}:|\gamma(1)-\gamma(0)| \geq \sigma\}, \quad Y_{\sigma}=\{[\gamma] \in \mathcal{C}:|\gamma(1)-\gamma(0)|=\sigma\} .
$$

This is straightforward, once one gets the existence of $H \in C^{0}\left([0,1] \times \mathcal{C} \backslash \mathcal{C}_{0}, \mathcal{C} \backslash \mathcal{C}_{0}\right)$ such that $H(0, x)=x \forall x \in \mathcal{C} \backslash \mathcal{C}_{0}, H(\tau, x)=x, \forall x \in X_{\sigma}, \forall \tau \in[0,1]$, and

$$
H\left(1, \mathcal{C} \backslash \mathcal{C}_{0}\right)=X_{\sigma}, \quad H\left(1, \mathcal{C}_{\sigma} \backslash \mathcal{C}_{0}\right)=Y_{\sigma}
$$

Step 3. cuplenght $\left(X_{\sigma}, Y_{\sigma}\right)=\operatorname{cuplenght}(E, \partial E)$, where $E$ is the closed unit disk bundle over the manifold $\mathrm{P}^{N-1}$ and $\partial E$ its boundary.

This is an immediate consequence of the fact that $\left(X_{\sigma}, Y_{\sigma}\right)$ is homeomorphic to $(E, \partial E)$.

Step 4. $\operatorname{cuplenght}(E, \partial E) \geq N-1$.

To prove this, let us observe that

$$
H^{q}\left(\mathbb{D}^{N-1}, \partial \mathbb{D}^{N-1}\right)= \begin{cases}0, & \text { if } q \neq N-1 \\ \mathbb{Z}_{2}, & \text { if } q=N-1\end{cases}
$$

Denoting by $\pi$ the canonical projection of $E$ in $\mathbb{P}^{N-1}$, thanks to the contractibility of $\mathrm{D}^{N-1}$ we see that

$$
\pi^{*}: H^{q}(E) \rightarrow H^{q}\left(\mathbb{P}^{N-1}\right) \text { is an isomorphism } \forall q \geq 0 .
$$

Since we are considering $\mathbb{Z}_{2}$-coefficients there are not problems with orientation, and by $\left[18\right.$, Corollary 5.7.18] the fiber bundle pair $\left((E, \partial E), \mathbb{P}^{N-1},\left(\mathbb{D}^{N-1}, \partial \mathbb{D}^{N-1}\right), \pi\right)$ has a unique orientation cohomology class $\zeta^{N-1}$ with dimension $N-1$. Then, by Thom isomorphism Theorem [18, Theorem 5.7.10] the homomorphism

$$
\Phi: H^{1}\left(\mathbb{P}^{N-1}\right) \rightarrow H^{q+N-1}(E, \partial E)
$$

given by $\Phi(z)=\pi^{*}(z) \cup \zeta^{N-1}$ is an isomorphism for any $q \geq 0$. From this fact and from A.5) we deduce that

$$
\operatorname{cuplenght}(E, \partial E) \geq \operatorname{cuplenght}(E) .
$$

Finally, using A.5 and standard results in literature (see e.g. [18]),

$$
\operatorname{cuplenght}(E)=\operatorname{cuplenght}\left(\mathbb{P}^{N-1}\right)=N-1 .
$$




\section{REFERENCES}

[1] V.Benci, D.Fortunato, F.Giannoni: Geodesics on static Lorentz manifolds with convex boundary, Proceedings on Variational Methods in Hamiltonian Systems and Elliptic Equations, Pitman Research Notes 243, (1990), 21-41.

[2] S. V. Bolotin and V. V. Kozlov, Libration in systems with many degree of freedom, J. Appl. Math. Mech. 42 (1978), 256-261.

[3] W. Bos, Kritische Sehenen auf Riemannischen Elementarraumstücken, Math. Ann. 151 (1963), 431-451.

[4] M. P. do Carmo, Riemannian Geometry, Birkhäuser, Boston, 1992.

[5] R. Giambò, F. Giannoni, Minimal geodesics on manifolds with discontinuous metrics, J. London Math. Soc., 67 (2003) 527

[6] R. Giambò, F. Giannoni, P. Piccione, Orthogonal Geodesic Chords, Brake Orbits and Homoclinic Orbits in Riemannian Manifolds, Adv. Diff. Eq., 10, (2005), 931-960.

[7] F. Giannoni, Multiplicity of principal bounce trajectories with prescribed minimal period on Riemannian manifolds, Diff. Int. Eq. 6,1451-1480 (1993).

[8] F. Giannoni, A. Masiello: On the existence of geodesics on stationary Lorentz manifolds with convex boundary, J. Funct. Anal. 101, 340-369 (1991).

[9] F. Giannoni, P. Majer, On the Effect of the Domain on the Number of Orthogonal Geodesic Chords, Diff. Geom. Appl. 7 (1997), 341-364.

[10] H. Gluck, W. Ziller, Existence of Periodic Motions of Conservative Systems, in "Seminar on Minimal Surfaces" (E. Bombieri Ed.), Princeton University Press, 65-98, 1983.

[11] E. Fadell, and S. Husseini, Relative cohomological index theories, Adv. Math 64 1-31, 1987.

[12] G. Fournier, and M. Willem, Multiple solutions of the forced double pendulum equation, Ann. Inst. H. Poincaré, Analyse non Lineaire 6 (suppl.) 259-281, 1989.

[13] D. Gilbarg, N. S. Trudinger, Elliptic partial differential equations of second order, Springer-Verlag, Berlin, (1983)

[14] Y. Long, D. Zhang, C. Zhu, Multiple brake orbits in bounded convex symmetric domains, Adv. Math. 203 (2006), no. 2, 568-635.

[15] Y. Long, C. Zhu, Closed characteristics on compact convex hypersurfaces in $\mathbb{R}^{2 n}$ Ann. of Math. (2) 155 (2002), no. 2, 317-368.

[16] L. Lusternik, L. Schnirelman, Methodes Topologiques dans les Problemes Variationelles, Hermann, 1934.

[17] H. Seifert, Periodische Bewegungen Machanischer Systeme, Math. Z. 51 (1948), 197-216.

[18] E. H. Spanier, Algebraic Topology, Springer-Verlag, New York, 1989.

[19] A. Szulkin, An Index Theory and Existence of Multiple Brake Orbits for star-shaped Hamiltonian Systems, Math. Ann. 283 (1989), 241-255.

[20] A. Weinstein, Normal modes for non-linear Hamiltonian systems, Inv. Math. 20 (1973), 47-57.

DIPARTIMENTO DI MATEMATICA E INFORMATICA

UNIVERSITÀ DI CAMERINO

ITALY

E-mail address: roberto.giambo@unicam.it, fabio.giannoni@unicam.it

DEPARTAMENTO DE MATEMÁticA

INSTITUTO DE MATEMÁtICA E ESTATÍSTICA

UNIVERSIDADE DE SÃo PAULO

BRAZIL

E-mail address: piccione.p@gmail.com 